NBER WORKING PAPER SERIES

\title{
COMMONALITY IN CREDIT SPREAD CHANGES: DEALER INVENTORY AND INTERMEDIARY DISTRESS
}

\author{
Zhiguo He \\ Paymon Khorrami \\ Zhaogang Song \\ Working Paper 26494 \\ http://www.nber.org/papers/w26494 \\ NATIONAL BUREAU OF ECONOMIC RESEARCH \\ 1050 Massachusetts Avenue \\ Cambridge, MA 02138 \\ November 2019
}

We are grateful to Yu An, Jennie Bai, Jack Bao, Florian Nagler, Yi Li, Arvind Krishnamurthy, Fabrice Tourre, and Alex Zhou, as well as seminar participants at Deakin University, Fordham University, Georgetown University, Johns Hopkins University, Monash University, University of Maryland, University of Illinois Urbana-Champaign, and University of Melbourne for helpful discussions. Zhiguo He acknowledges financial support from the Fama-Miller Center at the University of Chicago, Booth School of Business. Zhaogang Song is grateful to the Supplemental Research Support Fund of the Johns Hopkins Carey Business School. The views expressed herein are those of the authors and do not necessarily reflect the views of the National Bureau of Economic Research.

NBER working papers are circulated for discussion and comment purposes. They have not been peer-reviewed or been subject to the review by the NBER Board of Directors that accompanies official NBER publications.

(C) 2019 by Zhiguo He, Paymon Khorrami, and Zhaogang Song. All rights reserved. Short sections of text, not to exceed two paragraphs, may be quoted without explicit permission provided that full credit, including $(\odot$ notice, is given to the source. 
Commonality in Credit Spread Changes: Dealer Inventory and Intermediary Distress

Zhiguo He, Paymon Khorrami, and Zhaogang Song

NBER Working Paper No. 26494

November 2019

JEL No. G12,G22,G23

\begin{abstract}
$\underline{\text { ABSTRACT }}$
Two intermediary-based factors - a broad financial distress measure and a dealer corporate bond inventory measure - explain about 50\% of the puzzling common variation of credit spread changes beyond canonical structural factors. A simple model, in which intermediaries facing margin constraints absorb supply of assets from customers, accounts for the documented explanatory power and delivers further implications with empirical support.

First, whereas bond sorts on margin-related variables (credit rating and leverage) produce monotonic patterns in loadings on intermediary factors, non-margin-related sorts produce no pattern. Second, dealer inventory co-moves with corporate-credit assets only, whereas intermediary distress co-moves even with non-corporate-credit assets. Third, dealers' inventory increases, and bond prices decline, in response to instrumented bond sales by institutional investors, using severe downgrades ("fallen angels") and disaster-related insurance losses as IVs.
\end{abstract}

\author{
Zhiguo He \\ University of Chicago \\ Booth School of Business \\ 5807 S. Woodlawn Avenue \\ Chicago, IL 60637 \\ and NBER \\ zhiguo.he@chicagobooth.edu \\ Paymon Khorrami \\ Imperial College Business School \\ South Kensington Campus \\ London, SW7 2AZ, UK \\ p.khorrami@imperial.ac.uk
}

\author{
Zhaogang Song \\ The Johns Hopkins Carey \\ Business School \\ 100 International Drive \\ Baltimore, MD 21202 \\ zsong8@jhu.edu
}




\section{Introduction}

The commonality in excess variation of credit spread changes beyond credit risk factors, as documented in Collin-Dufresne, Goldstein, and Martin (2001) (hereafter CGM), is one of the canonical puzzles in asset pricing of credit risk. A key feature of U.S. corporate bond market is that broker-dealers serve as intermediaries of almost all transactions and use their balance sheets to take inventory and absorb bond supply from clients. Meanwhile, the recent intermediary asset pricing literature has argued that intermediary constraints are important determinants of asset prices (see He and Krishnamurthy (2018) for a survey). In this paper, we provide novel evidence that two intermediary factors account for about half of the puzzling common variation in credit spread changes. These factors are (1) a distress measure that captures constraints of the entire intermediary sector and (2) an inventory factor that captures the inventory held by dealers specializing in corporate bonds.

To construct the dealer inventory factor, we use the enhanced TRACE database of corporate bond transactions with untruncated trade size and anonymous dealer codes. Our measure of dealer inventory is computed using cumulative order flows (in par value) of transactions between customers and dealers. Using transaction records to construct inventory poses several practical difficulties, such as the unobservable level of dealers' bond inventory at the beginning of our sample period (2005:Q1), changes of dealer inventory unrelated to transactions (such as bond expiration), and missing primary market transactions from issuing firms to underwriting dealers. We address these issues carefully and then construct a quarterly measure of inventory by aggregating cumulative order flows of all dealers.

Our measure of intermediary distress combines two existing measures that have be shown to capture the severity of broad intermediation frictions. The first is a balance sheet leverage measure proposed by He, Kelly, and Manela (2017) (hereafter HKM) for bank holding companies of primary dealers recognized by the Federal Reserve Bank of New York (FRBNY); and the second is market-price-based "noise" measure proposed by Hu, Pan, and Wang (2013) (hereafter HPW), i.e., the root mean squared distance between the market yields of Treasury securities and the hypothetical yields implied from yield curve models. Our measure of intermediary distress is computed as the first principal component of these two measures, meant to parsimoniously capture the capital constraints on the aggregate intermediary sector.

Following CGM, our analysis starts by extracting residuals of individual-bond time series regressions of credit spread changes on seven structural factors. We assign each of the residual series into one of 15 cohorts based on time-to-maturity and rating, compute an average residual for each cohort, and extract the principal components of these 15 cohortlevel residuals. Similar to CGM, but with a comprehensive data set of corporate bond 
transactions in recent years, we find that about 80 percent of the variation can be explained by the first PC, indicating a large systematic component that is not captured by structural credit factors.

As one of the main contributions of this paper, we link our two intermediary factors the intermediary distress and dealer inventory factors - to this common variation of credit spread changes. We find that the two intermediary factors explain $53 \%$ of the variation of the first PC. More broadly, intermediary distress and dealer inventory explain $48 \%$ of the total variation of credit spread residuals. About $2 / 3$ of this explanatory power attributable to intermediary distress and $1 / 3$ to dealer inventory. Economically, one-standard-deviation increases of dealer inventory and intermediary distress are associated with quarterly credit spread increases of about 3-30 and 5-60 basis points, respectively. We further show that the effect is monotonically decreasing in bond ratings for both intermediary factors, an important empirical pattern that is relevant to our later theoretical modeling.

This strong explanatory power is robust to many alternative specifications. ${ }^{1}$ Given widespread interpretations of common non-structural credit spread movements as liquidityrelated, we are particularly interested in robustness to including transaction-cost-based liquidity factors studied in Dick-Nielsen, Feldhütter, and Lando (2012) and Bao, Pan, and Wang (2011). For instance, in Section 3.2, we show that the change of illiquidity measure in Dick-Nielsen, Feldhütter, and Lando (2012) exhibit non-significant correlations with our two intermediary factors, and can only explain about $0.6 \%-3 \%$ of common variation of CGM residuals.

Given the low correlation between distress and inventory, our empirical results indicate a two-factor structure of the common unexplained credit spread variation. We hence present a simple two-agent equilibrium model with hedgers and intermediaries trading multiple assets. Hedgers should be thought of as an agglomeration of institutional investors that face liquidity shocks, e.g., insurance companies and pension funds. Intermediaries absorb supply of bonds coming from hedgers, but are limited in their liquidity provision by a balance sheet constraint due to margin or capital requirements. This model features a single dominant factor, the Lagrange multiplier on the balance sheet constraint, that governs all non-fundamental movements in asset prices, consistent with our empirical evidence and that of CGM.

This single factor is endogenously driven by two types of shocks - hedger liquidity shocks

\footnotetext{
${ }^{1}$ Our results are also robust to using cohorts based on maturity and leverage; excluding the 2008 financial crisis period; measuring dealer inventory by market value; using only large dealers' inventory; doing all analysis at the monthly frequency; matching the horizon of credit spread changes and innovations of intermediary factors; and controlling for the Adrian, Etula, and Muir (2014) measure, TED spread (difference between three-month Libor and T-bill rates), and Pástor and Stambaugh (2003) stock liquidity factor.
} 
and intermediary wealth shocks. Hedger liquidity shocks are like "supply shocks" in the sense that more bonds arrive onto intermediaries' balance sheets, lowering bond prices. Of course, hedger liquidity shocks are unobservable, so we use the model to argue that dealer inventory effectively captures these supply shocks. Intermediary wealth shocks are like "demand shocks" in the sense that balance sheet frictions are alleviated, which shifts out intermediaries' demand schedules. Intermediary wealth shocks are effectively captured by leverage, one of the building blocks for our intermediary distress factor. Thus, through the lens of our model, our empirical exercise amounts to estimating a supply-demand system with two types of non-fundamental shocks. Model-based regressions with dealer inventory and leverage reproduce the qualitative patterns of our time series regressions in empirical analysis, in particular the monotonic pattern of sensitivities for bonds grouped by rating, which is a key determinant in a bond's margin/capital requirement.

Guided by the model, we develop three sets of empirical tests to further understand the economic channel behind the strong effects of intermediary factors on credit spread changes. First, sorting bonds by any characteristic unrelated to margin/capital requirements should not produce any pattern in associations to our two intermediary factors. Indeed, sorting by two such variables, maturity and trading intensity (measured by the total dollar trading volume), produces no detectible pattern in the economic magnitude or statistical significance of regression coefficients on our intermediary factors, controlling for bond rating.

Second, we enlarge our tests to other assets. An extended model with heterogeneous, imperfectly-integrated intermediary trading desks (e.g., a corporate credit desk, a Treasury desk, a securitized product desk, and an equity options desk), each with their own margin constraint, suggests the following testable predictions regarding co-movement across asset classes. Corporate-credit assets should be sensitive to dealers' corporate bond inventory, or even inventory computed from a subset of corporate bonds ("spillover effects"); noncorporate-credit assets should be insensitive to such inventory ("segmentation effects"); and both types of assets should be sensitive to aggregate intermediary distress. Intuitively, inventory coming from different asset classes exacerbates desk-specific constraints independently, whereas aggregate distress shocks affect all desks' constraints.

We find empirical support for this line of reasoning. Results of two tests support the spillover effect within corporate-credit markets: the first using dealer inventory of high-yield bonds and investment-grade bonds separately to explain credit spreads of all bonds, and the second using dealer inventory of bonds to explain CDS spreads. In contrast, agency mortgage-backed securities (MBS), commercial mortgage-backed securities (CMBS), assetbacked securities (ABS), and S\&P 500 index options are insensitive to corporate bond inven- 
tory, reflecting potential segmentation effects. Despite these differential sensitivities to bond inventory, all non-corporate-credit assets, as well as CDS, are sensitive to our intermediary distress factor, consistent with HKM who show empirically that primary dealers behave as the common marginal investor across many asset classes.

Third, we seek to establish a link between dealer inventory and liquidity shocks hitting other investors, as posited by the model. We do this by first establishing that dealer inventory does respond to such supply shocks. Next, we ask to what degree dealer inventory captures supply shocks versus other shocks by re-running our credit spread analysis with liquidity shocks as an IV for inventory.

To provide corroborating evidence linking liquidity shocks to dealer inventory, we use eMAXX data to measure bond holdings by each of the three groups of institutional investors - insurance companies, mutual funds, and pension funds. Given insurance companies face regulatory constraints in holding low-rated bonds (Ellul, Jotikasthira, and Lundblad, 2011), we interpret bond downgrades as a liquidity shock. Indeed, based on both summary statistics and formal regressions controlling for various bond characteristics, we find that insurance companies decrease their holdings of downgraded bonds, especially those downgraded from investment-grade to high-yield - so-called "fallen angels" (Ambrose, Cai, and Helwege, 2008) - by about $\$ 0.67$ million, relative to the average of those that experience no rating change or are downgraded from some IG rating to a lower IG rating. Mutual funds and pension funds take some of the IG-to-IG downgraded bonds, but not "fallen angels". Conversely, dealers' inventories of "fallen angels" increase substantially in the quarter when bonds are downgraded, by about $\$ 1.61$ million.

We then push this idea further to construct two instrumental variables for bond supply to dealers. As the first IV, we take the fallen angels sold off by institutional investors. To (partially) address the potential confound that fundamental changes trigger sell-offs and simultaneously lower bond prices, we control for sell-offs of all downgraded bonds. Second, we obtain unexpected insured losses due to natural disasters to proxy for forced sell-offs by insurance companies. This has clearer-cut exogeneity than fallen angel liquidations, but it is more likely to be a weak IV, because selling corporate bonds may be only one of many ways for insurance companies to fund large insurance payments.

First-stage regressions show that a one-standard-deviation decrease in institutional holdings of fallen angels and increase in insured loss is associated with a 0.20-0.37 standard deviation increase in dealer inventory, with strong statistical significance for the former but weak for the latter. In second-stage regressions, we hence use regular robust standard errors for evaluating the significance of fallen angel sell-offs but the Anderson and Rubin (1949) 
Wald-test and Stock and Wright (2000) S-statistic (which are weak-instrument robust) for insured losses. The results show that dealer inventory increases, instrumented by fallen angel sell-offs, are highly significant in increasing credit spreads. When instrumented by insured losses, the p-values on dealer inventory range from $10 \%$ to $15 \%$ across bond groups, also consistent with the positive effect of dealer inventory on credit spread changes. Finally, the effect of dealer inventory using IVs is larger than that in the baseline analysis, potentially because our IVs mitigate the downward bias caused by unobserved demand shocks simultaneously affecting dealer inventory.

Related literature. This paper contributes primarily to empirical literatures on corporate credit risk and intermediary asset pricing. In the credit risk literature, the unexplained common variation of credit spread changes, first documented in Collin-Dufresne, Goldstein, and Martin (2001) (CGM), is a canonical puzzle in the context of structural models like Merton (1974) and Leland (1994). ${ }^{2}$ Related is the "credit spread puzzle" of Huang and Huang (2012). In view of these puzzles, attention has been paid to the role of market liquidity, e.g., due to search frictions à la Duffie, Gârleanu, and Pedersen (2005). For example, Longstaff, Mithal, and Neis (2005), Bao, Pan, and Wang (2011), and Bao and Pan (2013) show that illiquidity measures affect credit spreads and corporate bond returns. ${ }^{3} \mathrm{He}$ and Milbradt (2014) develop a theory where credit risk in Leland and Toft (1996) and He and Xiong (2012) interacts with the over-the-counter search liquidity, with satisfactory quantitative performance over business cycles shown in Cui, Chen, He, and Milbradt (2017).

In the broad intermediary asset pricing literature, Adrian, Etula, and Muir (2014) and He, Kelly, and Manela (2017) are the first to show that financial intermediary balance sheets have pricing power for large cross-sections of assets. Recent contributions include Du, Tepper, and Verdelhan (2017), Chen, Joslin, and Ni (2018), Siriwardane (2019), Boyarchenko, Eisenbach, Gupta, Shachar, and Van Tassel (2018), and Fleckenstein and Longstaff (2019). We connect these intermediary-centric literatures to the corporate credit literature, arguing particular intermediation frictions govern the puzzling commonality in bond price variation.

By invoking dealers' special role in providing corporate bond liquidity, our study is related

\footnotetext{
${ }^{2}$ Schaefer and Strebulaev (2008) show that structural models capture well the sensitivity of corporate bond returns to equity returns or hedge ratios, which may seem to conflict the negative implication of CGM given the intrinsic relation between returns and yield spread changes. Huang and Shi (2014) find that structural models indeed characterize well the hedge ratios for credit spread changes, but half of variations in credit spread changes are still unexplained even after including explanatory variables or specifications that are important in characterizing hedge ratios.

${ }^{3}$ Relatedly, Lin, Wang, and Wu (2011), Acharya, Amihud, and Bharath (2013), and de Jong and Driessen (2012) study the pricing of liquidity risk in corporate bond returns.
} 
to other studies zooming in on bond dealers. For example, Bao, O'Hara, and Zhou (2018) and Bessembinder, Jacobsen, Maxwell, and Venkataraman (2018) show that dealers face higher regulatory constraints post 2008 crisis, which impairs their liquidity provision. Schultz (2017) and Dick-Nielsen and Rossi (2018) find that dealer inventory capacity is constrained recently, while Di Maggio, Kermani, and Song (2017) and Choi, Shachar, and Shin (2019) document dealers' inventory changes during the 2008 crisis. Our study differs by connecting dealers' corporate bond inventory to market prices. ${ }^{4}$

A closely related study, Friewald and Nagler (2019) (FN), conducts a comprehensive analysis of how OTC trading frictions - inventory, search, and bargaining frictions - affect credit spread changes, showing that twelve measures of such frictions jointly explain $23 \%$ of the CGM PC1. Besides the magnitude difference that we explain $53 \%$ of the CGM PC1, there are several distinctions. Whereas FN are focused on explanatory power, we use the loading patterns of bond groups on intermediary factors as evidence of our model's margin mechanism. In terms of the economic framework, their focus is microstructure-level trading frictions, whereas ours is dealers' aggregate balance sheet. Accordingly, we add an intermediary distress factor and conduct analysis at the quarterly frequency, a time-horizon on which microstructure frictions are less likely to dominate (for example, Schultz (2017) and Goldstein and Hotchkiss (2019) show that half lives of temporary dealer inventory, potentially due to search frictions, are up to several months). Finally, we espouse a parsimonious twofactor model that has a clear supply-demand interpretation attached to our inventory and distress factors, which leads us to design new tests. For example, we consider various asset classes (CDS, MBS, CMBS, ABS, options) to study imperfectly integrated trading desks within a bank, and we use bond-level holdings of insurance companies, mutual funds, and pension funds to provide refined evidence on the supply-demand channels.

Our two-factor empirical framework is motivated by a simple intermediary-based model with margin constraints. See Brunnermeier and Pedersen (2008) and Garleanu and Pedersen (2011) for dynamic asset pricing models with exogenous margin/capital constraints and Biais, Hombert, and Weill (2017) for equilibrium under endogenous versions of such constraints. ${ }^{5}$ The innovation of our static model is to focus in detail on two types of shocks: (1) asset supply shocks in the vein of Ho and Stoll (1981) or Kondor and Vayanos (2019); and (2) intermediary

\footnotetext{
${ }^{4}$ Two recent studies on equity market, Carole, Hendershott, Charles, Pam, and Mark (2010) and Hendershott and Menkveld (2014) relate variations of bid-ask spreads and prices to the inventory positions of New York Stock Exchange specialists.

${ }^{5}$ Because in the data margin constraints - and hence the asset pledgeability - are arguably endogenous to asset fundamentals, most of literature on asset pricing with margin constraint has been theoretical, with Chen, Chen, He, Liu, and Xie (2019) being the notable exception who causally estimate the pledgeability premium by exploiting the dual-listed bonds in Chinese bond markets.
} 
wealth shocks in the vein of many standard intermediary asset pricing models à la He and Krishnamurthy $(2012,2013)$. In addition, to study non-bond asset classes empirically, we extend the model in a way that incorporates some market segmentation on the intermediary side, unlike classic segmented-market frameworks like Gromb and Vayanos (2002). This extension can be interpreted as an economy with asset-class-specific intermediaries, as in the slow-moving-capital stories of Mitchell, Pedersen, and Pulvino (2007) and Duffie (2010).

The paper is organized as follows. Section 2 explains our data and measure. Section 3 presents our main findings. Section 4 presents a simple intermediary model to interpret the findings and delivers further implications we test in Section 5. Section 6 concludes.

\section{Data and Measures}

In this section, we introduce the data sample of U.S. corporate bond transactions and construct the empirical measures used in our main analysis. We also introduce the data sample of institutional holdings and other asset classes used in further tests.

\subsection{Data of Corporate Bond Transactions}

Our sample of corporate bond transactions are from the enhanced Trade Reporting and Compliance Engine (TRACE) maintained by the Financial Industry Regulatory Authority (FINRA). ${ }^{6}$ The FINRA started to collect data of corporate bond transactions in July 2002 and disseminated them to the public in three phrases through early 2005. We choose the starting of our sample period to be 2005:Q1 when the last phase was finished, similar to Schultz (2017) and Dick-Nielsen, Feldhütter, and Lando (2012), and end the sample at 2015:Q2. Each transaction record contains the trade date, time, (untruncated) principal amount, CUSIP, price, an indictor of whether the trade is either between a customer and a dealer or between two dealers, trading capacity of dealers (principal or agent), trade direction, and an anonymous dealer identifier, among many other variables.

\footnotetext{
${ }^{6}$ The TRACE database covers all corporate bond transactions executed by broker dealers registered with the FINRA. The missing trades from the TRACE database are those executed on all-to-all trading platforms or exchanges such as the New York Stock Exchange's Automated Bond System. These trades account for a very small portion of total corporate bond trading volume, less than $1 \%$ in 1990 and $5 \%$ in 2014 according to reports of U.S. SEC (1992) and Bank for International Settlements (2016).
} 


\subsubsection{Data Filtering}

We first apply a number of filters to account for reporting errors and to assign each trade to the actual trading counterparties. In particular, we adjust the sample for trade corrections and cancelations within the same day as well as across days (usually known as "trade reversals"). We account for the duplicated reports of inter-dealer trades. Furthermore, we assign a trade to the dealer who executed this trade rather than the reporting dealer for give-up trades in which one respective reporting firm reports on behalf of one actual trading counterparty (e.g., a clearing firm reports on behalf of a correspondent firm) and for locked-in trades in which one reporting firm reports on behalf of both actual trading counterparties. The data sample after these basic adjustments will be used to construct our measures of dealer inventory, hence we denote it the "bond inventory sample."

To construct the baseline sample for studying variation of credit spreads, we merge the TRACE database with the Mergent Fixed Income Securities Database (FISD) that provides bond characteristics including the age, maturity, amount outstanding, credit rating, issuance amount, coupon information, and various bond features such as whether a bond is convertible, puttable, callable, and so on. We further merge the data with CRSP for equity price information and with Compustat for accounting information. We exclude those that cannot be matched and restrict our sample to senior unsecured bonds that are denominated in U.S. dollars, with a fixed coupon rate, with available credit rating, and without embedded options. $^{7}$ We further exclude bonds issued by financial firms or utility firms, as we shall consider leverage as an important structural model factor that has different implications for these firms. We also exclude bonds with issue size less than $\$ 10$ million. In terms of trades, we keep only secondary market trades by removing the those with P1 flag (primary market trades; see more explanation in Section 2.2.1) and those with the trading date before and at the bond offering date. We exclude trades of bonds with time-to-maturity less than 1 year and also those with trade size larger than the issue size.

Our main sample frequency is quarterly. For each bond $i$, we take the price $p_{i, t}$ of the last trade in a quarter $t$ to compute the standard yield-to-maturity, and then calculate its credit spread $c s_{i, t}$ by subtracting off the yield of the corresponding Treasury security. ${ }^{8}$ The quarterly changes of credit spreads are then $\Delta c s_{i, t}=c s_{i, t}-c s_{i, t-1}$. However, many corporate bonds do not trade every day, so that the calculated $\Delta c s_{i, t}$ is not necessarily based on two actual quarter-end prices. To avoid large deviations from actual quarterly changes, we

\footnotetext{
${ }^{7}$ Similar to Bao and Hou (2017), we keep the bonds with a make whole call provision.

${ }^{8}$ The Treasury yields is calculated based on the Gurkaynak, Sack, and Wright (2007) database of Treasury yields with linear interpolations between provided maturities whenever necessary.
} 
exclude a $\Delta c s_{i, t}$ observation if the actual number of days between the trade dates in quarter $t$ and $t-1$ is lower than 45 days or larger than 120 days. We also match the Treasury yield to the exact day of the trade used in each quarter in computing credit spread to eliminate any nonsynchronization issues and scale $\Delta c s_{i, t}$ to make it a 90-day change (see Bao and Hou (2017) for similar adjustments at the monthly frequencies). Finally, we remove upper $1 \%$ and lower $1 \%$ tails of the credit spread levels to avoid the influence of outliers and require a bond to have at least 4 years of consecutive quarterly observations of $\Delta c s_{i, t}$ to ensure an enough number of observations for regressions on structural model factors.

For the detailed step-by-step procedure of data filtering and the associated change in sample coverage, see Table A.1.

\subsubsection{Summary Statistics}

Table 1 reports the summary statistics of our baseline sample of credit spreads. We have 2584 distinct bonds issued by 653 firms, with a total of 55,938 observations at the bondquarter level. Around $35 \%$ of the observations are on high-yield bonds, defined as the Moody's crediting rating lower than BBB. ${ }^{9}$ The mean credit spread is $1.52 \%$ and $5.27 \%$ for investment-grade and high-yield bonds, with a standard deviation of $1.17 \%$ and $3.65 \%$, respectively. The average time-to-maturity is 9.78 years, with a higher mean for investmentgrade bonds at 10.85 years than for high-yield bonds at 6.78 years. For investment-grade bonds, average issuance size is about $\$ 600$ million, whereas high-yield bonds' is only about $\$ 400$ million. That is, lower rated firms tend to be smaller. The average coupon rates are lower for investment-grade $(5.87 \%)$ than high-yield bonds $(7.6 \%)$.

For comparison, Bao and Hou (2017) use a sample of about 10 years from July 2002 to December 2013. Because they focus on monthly frequency, they have a larger sample size with more than 230,000 bond-month observations and around 7000-9000 distinct bonds. FN also use a monthly dataset from January 2003 to December 2013. Their sample includes only 974 bonds with 45,000 bond-month observations, substantially smaller than that of Bao and Hou (2017) and both our quarterly and monthly samples (our monthly sample has 3324 bonds and more than 185,000 bond-month observations; see Table A.1).

\footnotetext{
${ }^{9}$ The sample and results remain little changed when we use the average of available Moody's, Standard \& Poor's, and Fitch ratings.
} 


\subsection{Intermediary Factors}

This section explains how we construct the two intermediary factors in our paper: dealer corporate bond inventory, and intermediary distress.

\subsubsection{Dealer Inventory}

Our measure of dealer inventory is computed using cumulative order flows between customers and dealers from the data of corporate bond transactions. As our objective is to study the balance sheet pressure imposed by aggregate dealer inventory rather than bond-specific characteristics, we use the "bond inventory sample" as defined in Section 2.1 that includes the whole set of corporate bond transactions. ${ }^{10}$

Using records of transactions to construct measures of inventory poses several practical difficulties, which we address carefully in a few steps. First, we have no data on the actual level of dealers' bond inventory at the beginning of our sample period (2005:Q1 2015:Q2). Accordingly, we construct the dealer inventory measure starting from 2002:Q3 when the TRACE data of corporate bond transactions first became available, but only use the inventory measure after 2005:Q1. With this "buffer" period of two and half years, the mismeasurement of dealer inventory starting from 2005:Q1 should be mitigated, in light of the evidence on half lives of dealer inventory being up to several months (e.g., Schultz (2017), Goldstein and Hotchkiss (2019)). The initial holdings should have matured and/or been unwound by dealers.

Second, we do not observe changes of dealer inventory unrelated to market transactions. If dealers hold a bond until its maturity, there is no transaction record indicating the expiration of the bond position. To deal with this issue, we calculate cumulative positions of all dealers for each bond, and from the date of the last transaction of a bond, we assume dealers' inventory of this bond turns zero at its maturity date and hence remove this amount of inventory on that date. ${ }^{11}$

Third, dealers who are active in the primary market are "selling" bonds around the issuance date, which should be excluded as we ignore the newly issued corporate bonds in our calculation. Starting March 1, 2010, the FINRA started to require member firms to report trades executed in the primary market, and an identifier is assigned on these trades. Hence we use the provided identifier to take out primary market trades executed after March

\footnotetext{
${ }^{10}$ This sample is a superset of the baseline sample of individual-bond credit spreads, as discussed in Section 2.1.2.

${ }^{11}$ Our procedure will miss those callable bonds that are being called before their maturity, though these callable bonds are removed from inventory at their times of maturity.
} 
1, 2010. For the sample before March 1, 2010, we remove trades of a bond executed before and on its offering date obtained from the Mergent FISD. This procedure should remove most of the primary market trades as underwriting dealers are expected to finish delivering bonds within a short period of time. ${ }^{12}$

After making these adjustments, we construct a quarterly measure of dealer inventory by aggregating cumulative order flows of all dealers as a whole with customers. We use the par value rather than market value to avoid the potential confounding effect of price changes when studying the effect of dealer inventory on bond prices. The quarterly log change of this measure, denoted $\Delta$ Inventor $^{A}$, is the baseline dealer inventory factor in our analysis of credit spread changes. We also construct separate measures of dealers' high-yield and investment-grade inventory for some specific tests, which will be discussed in detail later.

To the best of our knowledge, data on dealers' exact holding amounts of corporate bonds are unavailable. In addition to using transaction data to measure dealer inventory as we do, two data sources based on financial reporting also provide some crude information on dealers' security holdings. One is the FRBNY report on holdings of primary dealers, ${ }^{13}$ and the other is the Flow of Funds report on holdings of security broker-dealers, released by the Federal Reserve. ${ }^{14}$ Several differences and issues are worth discussing. First, the FRBNY began collecting primary dealers' holdings of corporate bonds as a separate asset class only starting April 3, 2013; its reported corporate bond positions prior to April 3, 2013 were extrapolated. ${ }^{15}$ Second, the FRBNY report only includes about 20 primary dealers, while the Flow of Funds series cover all brokers and dealers that submit information to the Securities and Exchange Commission through either the Financial and Operational Combined Uniform Single Report (FOCUS) or the Report on Finances and Operations of Government Securities (FOGS). However, the Flow of Funds series are the holding amounts of "corporate and foreign bonds" (FL663063005.Q) that include both corporate bonds and all other fixedincome securities such as private-label MBS.

Unlike inventory measures based on these two alternative data, our measure of dealer inventory only includes corporate bonds. Furthermore, our measure covers all dealers trading

\footnotetext{
${ }^{12}$ Before March 1, 2010, market participants were facing ambiguity regarding the definition of primary versus secondary market trades. Our procedure may eliminate some secondary market trades on the issuance day as well as keep some primary market trades after the issuance day.

${ }^{13}$ See https://www . newyorkfed.org/markets/gsds/search.html.

14 "Financial Accounts of the United States" (Z.1) at https://www.federalreserve.gov/apps/fof/ DisplayTable. aspx?t=1.130.

${ }^{15}$ Before April 3, 2013, only aggregate holdings that do not separate corporate bonds from securities issued by non-federal agencies (e.g., government-supported enterprises) are available. The FRBNY extrapolates corporate bond positions prior to April 3, 2013 using the composition of corporate bond holdings on that date.
} 
in corporate bonds, which is more than just the FRBNY primary dealers. We also exclude dealers without corporate bonds holdings, unlike the Flow of Funds, which does not separate holdings of corporate bonds from other securities. ${ }^{16}$

\subsubsection{Intermediary Distress}

To construct the intermediary distress factor, we combine the balance-sheet-based leverage ratio measure of the aggregate intermediary sector proposed by HKM and the market-pricebased "noise" measure proposed in HPW. The HKM leverage ratio, denoted Lev ${ }_{t}^{\mathrm{HKM}}$ for quarter $t$, is computed as the aggregate market equity plus aggregate book debt divided by aggregate market equity, using CRSP/Compustat and Datastream data, of the holding companies of primary dealers recognized by the FRBNY.

In measuring the change or innovation of the leverage ratio, we create the variable $\Delta \mathrm{NLev}_{t}^{\mathrm{HKM}}:=\left(\operatorname{Lev}_{t}^{\mathrm{HKM}}-\operatorname{Lev}_{t-1}^{\mathrm{HKM}}\right) \times \operatorname{Lev}_{t-1}^{\mathrm{HKM}}$, motivated by the nonlinear affect of intermediary constraints on asset prices implied from intermediary-based asset pricing models like He and Krishnamurthy (2013) and Brunnermeier and Sannikov (2014). Note that $\Delta$ NLev $_{t}^{\text {HKM }}$ weights the change of leverage ratio higher when its level is high.

The HPW "noise" measure is computed as the root mean squared distance between the market yields of Treasury securities and the hypothetical yields implied from yield curve models like that of Svensson (1994). ${ }^{17}$ Besides its obvious connection to our paper as corporate bonds are part of fixed-income securities, "noise" is widely used in the literature as a measure of "shortage of arbitrage capital" across various markets. The rationale is that relative value trading across various habitats on the yield curve is widely conducted at most investment banks and fixed-income hedge funds. Hence, a significant deviation of market yields from model-implied yields is a symptom of a lack of arbitrage capital, and importantly, "to the extent that capital is allocated across markets for major marginal players in

\footnotetext{
${ }^{16}$ The discrepancies in terms of asset and dealer coverage lead to differences between our dealer inventory measure and the other two alternative data sources. First, the magnitudes often diverge; for example, the total holding of primary dealers is about $\$ 250$ and $\$ 28$ billion at the end of 2007Q1 and 2014Q4, respectively, while about $\$ 91$ and $\$ 107$ billion from our series. Second, our level series of dealer inventory shows an expansion starting from early 2013 - which is consistent with increasing outstanding balance of corporate debt (see Figure 2) - but is absent in the two alternative measures. That being said, both our measure and the two alternative measures share a similar increasing trend from early 2003 up to mid-2007 and a large decline thereafter until 2012.

${ }^{17}$ The Svensson (1994) model is an extension of the yield curve model initially proposed in Nelson and Siegel (1987). These models are widely used in the academic literature and in practice to compute benchmark yield curves. For example, Gurkaynak, Sack, and Wright (2007) use them to construct Treasury yield curves that are regular inputs of the Federal Reserve's policy discussions and publications. Song and Zhu (2018) discuss the use of these models by the Federal Reserve in evaluating offers submitted in auctions that executed the purchases of Treasury securities for quantitative easing.
} 
the market, this symptom applies not only to the Treasury market, but also more broadly to the overall financial market" (see HPW). The quarterly change of the HPW noise measure (in basis points) is denoted $\Delta$ Noise.

Our measure of intermediary distress, denoted as $\Delta$ Distress, is defined as the first principal component of $\Delta \mathrm{NLev}_{t}^{\mathrm{HKM}}$ and $\Delta$ Noise. The former is constructed mainly using balance sheet information of financial intermediaries, while the latter is based on prices. Combining the two leads to a parsimonious measure of the capital constraints on the aggregate inter-

mediary sector. As shown later in Section 3.3, both $\Delta \mathrm{NLev}_{t}^{\mathrm{HKM}}$ and $\Delta$ Noise contribute a nontrivial fraction of the explanatory power of $\Delta$ Distress for credit spread changes.

\subsubsection{Summary Statistics}

To gauge the variation of the two intermediary factors, Figure 1 plots the quarterly time series of $\Delta$ Inventory ${ }^{A}$ and $\Delta$ Distress (both scaled to have zero mean and unit variance) in the top and middle panels, respectively. Dealer inventory has comparable frequent variation across different sub-periods of the sample, whereas intermediary distress exhibits extreme variation in the 2008 crisis but mild variation otherwise. Importantly, although $\Delta$ Inventory $^{A}$ does show large negative values in the 2008 crisis, similar to $\Delta$ Distress, the two factors exhibit orthogonal variation to a large extent. Table 2 shows the correlation between $\Delta$ Inventory ${ }^{A}$ and $\Delta$ Distress is only -0.16 , with an intuitive negative sign because sales by a highly-levered dealer sector should lead to a lower inventory, though statistically insignificant.

The bottom panel of Figure 1 plots the quarterly time series of $\Delta \mathrm{NLev}^{\mathrm{HKM}}$ and $\Delta$ Noise that are used to construct our measure of intermediary distress. These two series line up with each other well, though $\Delta$ Noise led $\Delta \mathrm{NLev}^{\mathrm{HKM}}$ by a quarter in plummeting during the 2008 crisis. The correlation between them, from Table 2, is 0.83 . Our measure $\Delta$ Distress, as the first principal component of them, captures $70 \%$ of the total variation.

Table 2 also reports the correlation of our intermediary factors with the change of VIX that is widely used as an indicator of financial market stress. We find that $\Delta$ Distress has a significant positive correlation with $\triangle$ VIX, about 0.36 . In contrast, the correlation of $\Delta$ Inventory $^{A}$ with $\Delta V I X$, though with an intuitive negative sign, is less than $10 \%$ and statistically insignificant, implying that dealer inventory is largely orthogonal to these popular measures of market stress. In addition, both of our intermediary factors have low correlations with the illiquidity factor $\triangle$ ILiq of corporate bond trading of Dick-Nielsen, Feldhütter, and Lando (2012). We shall control for $\Delta V I X$ and $\Delta$ ILiq in studying the effects of intermediary factors on credit spread changes. We now turn to introduce additional measures and controls variables. 


\subsection{Structural Factors and Control Variables}

Following CGM, we consider seven determinants, motivated from the Merton (1974) model, of credit spread changes: firm leverage $L e v_{i, t}$, 10-year Treasury interest rate $r_{t}^{10 y}$, square of 10-year Treasury interest rate $\left(r_{t}^{10 y}\right)^{2}$, slope of the term structure Slope $_{t}$ measured as the difference between 10-year and 2-year Treasury interest rates, S\&P 500 return $\operatorname{Ret}_{t}^{S P}$, a jump factor $J_{u m p}$ based on S\&P 500 index options, and VIX . The firm leverage $L e v_{i, t}$ is computed as the book debt over the sum of the book debt and market value of equity. The book debt is defined as the sum of "Long-Term Debt - Total" and "Debt in Current Liabilities - Total" from Compustat, whereas the market value of equity is equal to the number of common shares outstanding times the share price from CRSP. The debt data from Compustat are available at the quarterly frequency, and we follow the literature to assume that such balance sheet information becomes available with a lag of one quarter (Bao and Hou, 2017).

The interest rate factors $r_{t}^{10 y},\left(r_{t}^{10 y}\right)^{2}$, and Slope $t$ are calculated based on the Gurkaynak, Sack, and Wright (2007) database of Treasury yields (in percent). The S\&P 500 return $\operatorname{Ret}_{t}^{S P}$ is from CRSP, while the $V I X_{t}$ is from CBOE. The jump factor Jump $p_{t}$ is computed based on at-the-money and out-of-the-money options on the S\&P 500 index, from OptionMetrics (see CGM for details on the procedure).

\subsection{Institutional Holdings of Corporate Bonds}

We obtain data on institutional investors' holdings of corporate bonds from the survivorshipbias-free Lipper eMAXX database of Thomson Reuters. This data set contains quarter-end security-level corporate bond holdings of insurance companies, mutual funds, and pension funds in North America (based on where the holder is located). Data on insurance companies' holdings are based on National Association of Insurance Commissioners (NAIC) annual holdings files and quarterly transaction reports to the state insurance commissioners. Data on mutual fund holdings are obtained from Lipper, which is owned by Thomson Reuters, covering over $90 \%$ of the mutual fund universe. Data on pension fund holdings are from state and local municipal pension funds and large private pension funds who voluntarily submit data to Thomson Reuters (see Cai, Han, Li, and Li (2019), Bo and Victoria (2015), and Manconi, Massa, and Yasuda (2012) among others for further details). We use the eMAXX holdings over 2005:Q1 - 2015:Q2, with information on bond characteristics such as historical outstanding balance and credit rating by matching to FISD based on the CUSIP number.

Figure 2 provides a summary of the eMAXX institutional holdings, as well as dealer 
inventories. In particular, the top panel plots quarterly time series of the holding amount by institutional investors (including mutual funds, pension funds, and insurance companies) based on eMAXX data and by dealers based on TRACE data, as well as the aggregate outstanding balance of U.S. corporate debt securities based on the Federal Reserve's Flow of Funds, in \$trillions of principal value. ${ }^{18}$ The dollar (par) value of holdings has seen a significant increase from $\$ 1.3$ trillion to $\$ 2.7$ trillion, with much of the increase coming after plummeting in the 2008 crisis. The rise of holdings is strongest in mutual funds. At the same time, there has been a sizable expansion of the whole corporate bond market, as the total outstanding balance increased from less than $\$ 5$ trillion to more than $\$ 8$ trillion. The bottom panel plots quarterly time series of the fraction of U.S. corporate debt securities held by institutional investors, by dealers, and by both, in percent. The fraction steadily accounts for $25-35 \%$ of the aggregate outstanding balance.

\subsection{Yield Spreads and Returns of Other Asset Classes}

Our analysis also uses yield spreads and returns of a host of other asset classes including CDS, agency MBS, CMBS, ABS, and equity options. We obtain CDS quotes on individual U.S. corporations denominated in U.S. dollars from Markit. We use 1-year, 5-year, and 10-year CDS contracts with modified restructuring (MR) clauses, among which 5-year CDS are the most traded. We match the CDS data with equity information from CRSP and accounting information from Compustat. For each entity, we construct quarterly series of CDS spreads using the last quotation in every quarter.

We obtain series of yield spreads of agency MBS, CMBS and ABS from major Wall Street dealers. Specifically, we use (option-adjusted) yield spreads of agency MBS based on the liquid "to-be-announced" (TBA) contracts of 15-year and 30-year production-coupon Fannie Mae and Freddie Mac MBS (see Gabaix, Krishnamurthy, and Vigneron (2007) and Gao, Schultz, and Song (2017) among others for details of TBA contracts and option-adjusted spreads). We use the Barclays yield spreads of non-agency 10-year CMBS of three AAArating groups, Super Duper Senior (Duper), mezzanine (AM), and junior (AJ). ${ }^{19}$ We also use yield spreads of 5-year AAA-rated ABS on fixed-rate credit card loans and 3-year ABS

\footnotetext{
${ }^{18}$ Specifically, the aggregate outstanding balance is the sum of the outstanding debt securities by nonfinancial corporate business, U.S.-chartered depository institutions, foreign banking offices in the U.S., finance companies, security brokers and dealers, and holding companies in the "L.208 Debt Securities" series.

${ }^{19}$ These different groups differ in terms of credit enhancement. Moreover, since CMBS usually have restrictions on prepayment and are different from residential-loans backed agency MBS, we use the yield spreads for CMBS but option-adjusted spreads for agency MBS. See Manzi, Berezina, and Adelson (2016) for further details.
} 
on fixed-rate prime auto loans of AAA, A, and BBB ratings.

In addition, we use monthly returns of portfolios of $\mathrm{S} \& \mathrm{P} 500$ index options sorted on moneyness and maturity from Constantinides, Jackwerth, and Savov (2013). These portfolios are leverage-adjusted in that each option portfolio is combined with risk-free account to achieve a targeted market beta of one. A leverage-adjusted call option portfolio consists of long positions in calls and some investment in the risk-free account, while a leverageadjusted put portfolio consists of short positions in puts and more than $100 \%$ investment in the risk-free account. For the convenience of interpretation, we take the negative of the put portfolio return. The holding period of these option portfolios is a month regardless of the target maturity that is 30,60 , or 90 days. We use the 30-day maturity to match the holding period precisely, but results are similar using 60-day and 90-day maturities.

\section{Main Empirical Results}

We discuss the main empirical results in this section. We first replicate the exercise in CGM and show that the strong commonality persists in the U.S. corporate bond market in the past decade. Our two intermediary-based variables, intermediary distress and dealer inventory, can explain more than half of the latent common factor. Especially important to our paper is the fact that sensitivities to these intermediary-based factors are monotone in credit risk (i.e., ratings), a pattern that is robust to many other alternative specifications.

\subsection{Baseline Analysis}

\subsubsection{Commonality of Credit Spread Changes}

To study the effects of intermediary constraints on corporate bond pricing, we follow CGM and run a time series regression for each bond $i$ :

$$
\begin{aligned}
\Delta c s_{i, t}= & \alpha_{i}+\beta_{1, i} \times \Delta \operatorname{Lev}_{i, t}+\beta_{2, i} \times \Delta V I X_{t}+\beta_{3, i} \times \Delta \operatorname{Jump}_{t} \\
& +\beta_{4, i} \times \Delta r_{t}^{10 y}+\beta_{5, i} \times\left(\Delta r_{t}^{10 y}\right)^{2}+\beta_{6, i} \times \Delta \text { Slope }_{t}+\beta_{7, i} \times \operatorname{Ret}_{t}^{S P}+\varepsilon_{i, t},
\end{aligned}
$$

by which an estimate of each regression coefficient for each bond is obtained. To avoid asynchronicity issues, in running this regression for bond $i$, we match the dates of any structural regressors available at daily frequency (e.g., $V I X_{t}$ ) to the dates of measured credit spreads for bond $i$. Similar to the empirical procedure of CGM, we assign each bond into one of 15 cohorts based on time-to-maturity and rating, and then report the regression results at 
the cohort-level. Panel A of Table 3 shows that the sample size is fairly homogenous across maturity groups but heterogeneous across rating groups.

Panel A reports the regression results. Following CGM, we report the average regression coefficients across bonds within each cohort, with associated t-statistics computed as the average coefficient divided by the standard error of the coefficient estimates across bonds. The dependence of credit spread changes on the factors is as expected based on structural frameworks. For example, credit spreads significantly increase with firm leverage and volatility, and decrease with the risk-free rate and the stock market return. The effects of convexity and slope of the term structure are less consistently significant: convexity shows some negative significance for long-maturity bonds and slope of the term structure shows some positive significance for short-maturity bonds, but the coefficients on both switch signs in certain cohorts. The jump factor shows negative significance for most of the medium and long term bonds. Finally, the mean adjusted $R^{2}$ is about $30-40 \%$ for bonds rated equal to or above $\mathrm{BBB}$ and about $55 \%$ for bonds rated equal or below $\mathrm{BB}$.

Most importantly, there is a strong common factor structure of the regression residuals. Panel B of Table 3 reports the principal component analysis of the 15 series of regression residuals. The residual series $\varepsilon_{g, t}$ of each cohort $g$ are computed as the average of regression residuals $\varepsilon_{i, t}$ across bonds $i$ in the cohort $g$. Over $80 \%$ of the variation can be explained by the first PC, whereas the second PC explains an additional 6\% (the third PC only explains less than $2 \%$, so we only report the first two PCs). Credit spread changes contain a large systematic component that is not captured by structural model factors, as pointed out by CGM.

Moreover, the last column of Panel A reports the variation of residuals for each cohort $g, \varepsilon_{g}^{v a r}\left(=\sum_{t}\left(\varepsilon_{g t}-\bar{\varepsilon}_{g}\right)^{2}\right.$ with $\bar{\varepsilon}_{g}$ the time series mean of $\left.\varepsilon_{g t}\right)$, as a fraction of the total variation of the 15 cohorts $\sum_{g=1}^{15} \varepsilon_{g}^{v a r}$. We observe that the BB and B cohorts account for the majority of the total variation, about $86 \%$. That is, although the structural factors can explain more than $50 \%$ of the raw credit spread changes in these two cohorts, what remains to be explained is still large compared to higher-rated cohorts.

It is worth comparing our data sample and results with those of two closely related studies, CGM and FN. In term of data sample, CGM use a 10-year monthly sample from July 1988 to December 1997 with a total of 688 bonds and dealer quote prices, while FN also use a 10-year monthly sample but from January 2003 to December 2013 with a total of 974 bonds and actual transaction prices. We use a 10-year quarterly sample from 2005:Q1 to 2015:Q3 with a total of 2584 bonds and actual transaction prices.

In terms of the overall explanatory power, the average adjusted $R^{2}$ is about $25 \%$ and 
$22 \%$ in CGM and FN, respectively, but about $45 \%$ in our study. The much higher adjusted $R^{2}$ is likely because we use a 10-year quarterly sample as opposed to the 10-year monthly sample in the other two studies (indeed, in the monthly regressions reported in Table 7, the average adjusted $R^{2}$ drops to $26 \%$.). Most importantly, the fraction of the total unexplained variance of regression residuals that can be accounted for by the first PC is $76 \%$ in CGM and $82 \%$ in our study, but only $48 \%$ in FN. Overall, all three studies confirm a strong common factor structure for the credit spread changes beyond those driven structural factors, though our paper and CGM document a much stronger commonality than FN. ${ }^{20}$

A possible explanation for this discrepancy, as proposed by FN, is that CGM's use of dealer quotes instead of actual transaction prices "potentially works against their conclusion regarding the magnitude of the latent factor" (page 8 in FN). Our results seem inconsistent with this conjecture given that actual transaction prices are also used in our analysis. The same strong comovement persists in our monthly sample: the PC1 accounts for $76 \%$ of the variation of the 15 portfolios of corporate bonds, as shown in Table 7 in the next section, exactly matching the finding of CGM.

\subsubsection{Effect of Intermediary Factors on Common Credit Spread Changes}

We study the effect of intermediary factors on common credit spread changes based on the following time series regressions:

$$
\varepsilon_{g, t}=\alpha_{g}+\beta_{1, g} \Delta \text { Inventory } y_{t}^{A}+\beta_{2, g} \Delta \text { Distress }_{t}+u_{g, t},
$$

where $\varepsilon_{g, t}$ is the average residual of cohort $g(=1, \ldots, 15)$. Panels A and B of Table 4 report univariate regressions on dealer inventory and intermediary distress, respectively, and Panel C reports bivariate regressions. Dealer inventory and intermediary distress both comove positively with residuals of credit spread changes. The statistical significance of dealer inventory is weak in univariate regressions, ${ }^{21}$ but strong in joint regressions, whereas inter-

\footnotetext{
${ }^{20}$ In terms of individual explanatory variables, leverage, volatility, risk-free rate, and stock market return exhibit uniformly consistent explanatory power, across all three studies, while the significance of convexity, slope of the term structure, and jump factor are weaker and exhibits different patterns. For example, the coefficient on slope of the term structure is significantly positive in $\mathrm{FN}$, in contrast to the overall negative significance documented in CGM and for short-term bonds in our study. The coefficient on jump factor is significantly positive in CGM, weakly positive in FN, but significantly negative for medium- to long-term bonds in our study.

${ }^{21}$ This is likely due to the unbalanced number of bonds assigned into different cohorts. Indeed, for cohorts based on firm leverage with a much more balanced number of observations in different cohorts, as used in Table 6 of next section, the statistical significance of both intermediary factors is strong in univariate regressions.
} 
mediary distress shows strong statistical significance in both univariate and joint regressions.

Economically, co-movement between spreads and intermediary factors is large. Since we have standardized the intermediary factors in regressions, the joint regression in Panel $\mathrm{C}$ of Table 4 implies that a one-standard-deviation increase of dealer inventory is associated with a quarterly increase of about 3-30 basis points in bond yields. For intermediary distress, this number is about $5-60$ basis points.

The effect of both intermediary factors is stronger for lower-quality bonds. For example, the coefficients on dealer inventory (intermediary distress) monotonically increase from 0.011 to 0.278 (from 0.048 to 0.499 ) for medium-term bonds, when the rating goes from AA and above down to $\mathrm{B}$ and below. This monotonic pattern is reminiscent of the principal component loadings: lower-rated bond residuals have higher loadings on PC1 in Table 3.

Finally, to evaluate the overall explanatory power of the intermediary factors on credit spread changes, we compute the fraction of the total variation of residuals that is accounted for by $\Delta$ Inventory ${ }^{A}$ and $\Delta$ Noise. In particular, for each of the 15 time series regressions, we can compute the total variation of credit spread residuals $\varepsilon_{g}^{v a r}$ as above and also the variation $u_{g}^{v a r} \equiv \sum_{t}\left(u_{g, t}\right)^{2}$ that cannot be explained by the two intermediary factors. For each of the three maturity groups and all 15 groups, we compute the fraction of variation explained by the two intermediary factors as

$$
\mathrm{FVE}_{G}=1-\frac{\sum_{g \in G} u_{g}^{v a r}}{\sum_{g \in G} \varepsilon_{g}^{v a r}}
$$

where $G \in\{$ short, medium, long, all $\}$. As reported in the last column of Table 4, the two intermediary factors explain $38 \%, 55 \%$, and $50 \%$ of the total variation of residuals of credit spread changes for short, medium, and long term bonds, respectively, and about $48 \%$ for all bonds. Similar calculations for dealer inventory and intermediary distress separately show that $2 / 3$ of this explanatory power can be attributed to intermediary distress and $1 / 3$ to dealer inventory, which is consistent with the correlations of these two factors and the PC1 reported in the last row of Table 3.

In sum, our baseline analysis shows that dealer inventory and intermediary distress have significant positive effects on common credit spread changes. The effects monotonically decrease with bond ratings. The two factors together account for about half of the unexplained total variation of credit spread changes, with one third and two thirds attributable to dealer inventory and intermediary distress, respectively. 


\subsection{Microstructure Liquidity Factor and Credit Spread Changes}

Motivated by the finding of CGM, many studies have focused on microstructure liquidity factors such as bid-spread and trading intensity as a potential driver of credit spreads (e.g., Chen, Lesmond, and Wei (2007) and Bao, Pan, and Wang (2011)). It is hence instructive to understand how much of credit spread changes at the quarterly frequency can be explained by these microstructure-level factors in comparison to our intermediary factors. We use the illiquidity measure of Dick-Nielsen, Feldhütter, and Lando (2012) that is an equally weighted sum of the Amihud (2002) measure of price impact, the Feldhütter (2012) measure of roundtrip cost, and respective daily standard deviations of these two measures. That is, their illiquidity measure captures trading illiquidity due to price impact and transaction costs, as well liquidity risk.

We first note that the change of illiquidity measure in Dick-Nielsen, Feldhütter, and Lando (2012) exhibit non-significant correlations with our two intermediary factors, as shown in Table 2. Second, Table 5 reports quarterly time series regressions of each of the 15 credit spread residuals on $\Delta$ ILiq, both in univariate regressions (Panel A) and in multivariate regressions along with our two intermediary factors (Panel B), respectively. The results show that $\Delta$ ILiq mainly adds to the explanatory power (adjusted $R^{2}$ ) of high-rated cohorts but not low-rated cohorts, and its explanatory power is quite small. In particular, Panel A shows that $\triangle \mathrm{ILiq}$ accounts for about $3 \%$ of the total variation of residuals of credit spread changes (and significantly positive only for high-rated cohorts). ${ }^{22}$ In panel B, we observe that relative to our baseline with two intermediary factors, $\Delta$ ILiq only increases the explained fraction by $0.6 \%$ (from $48.2 \%$ to $48.8 \%$ ). ${ }^{23}$

\footnotetext{
${ }^{22}$ The influential measure of corporate bond market illiquidity proposed by Bao, Pan, and Wang (2011) is available at the monthly frequency but only up to 2009. We do not expect any material difference using either of these two measures in our analysis, as the correlation between them is 0.95 and 0.94 at monthly and quarterly frequencies over 2003 - 2009 when the Bao, Pan, and Wang (2011) measure is available. Indeed, we conduct monthly regressions using the Bao, Pan, and Wang (2011) measure over 2005 - 2009, reported in Panel B of Table A.6, and find the similar qualitative result that the change of illiquidity mainly affects credit spread changes of high-rated bonds, just like Table 5 that uses the Dick-Nielsen, Feldhütter, and Lando (2012) measure. This pattern is also found in Bao, Pan, and Wang (2011).

${ }^{23} \mathrm{In}$ an alternative approach, we add $\Delta \mathrm{ILiq}$ as an explanatory variable to the individual-bond regression (1). Consistent with the pattern in Table 5 , it mainly adds to the explanatory power (adjusted $R^{2}$ ) of highrated cohorts but not low-rated cohorts. Our two intermediary factors explain $45 \%$ of the total variation of residuals, only slightly lower than the $48 \%$ in the baseline analysis; and this $3 \%$ difference merely reflects the $3 \%$ of explanatory power of $\Delta \mathrm{ILiq}$ alone reported in Panel A in Table 5.
} 


\subsection{Robustness Checks}

In this section, we provide further results that elaborate on the explanatory power of intermediary factors for credit spread changes and confirm robustness (additional robustness results are in Appendix A).

First, Table 6 reports the results using 15 cohorts based on time-to-maturity and firm leverage. Similar to CGM, we set the breakpoints of leverage to obtain a relatively homogeneous distribution of bonds across cohorts. ${ }^{24}$ Panel B reports a principal component analysis on the 15 residual series, showing a similar strong common variation, with the PC1 accounting for $78 \%$ of the total unexplained variation of credit spread changes. Panel $\mathrm{C}$ reports the quarterly bivariate series regressions of each of the 15 residuals on dealer inventory and intermediary distress. Credit spread changes co-move significantly and positively on intermediary factors, with the loadings monotonically increasing with leverage. Compared with the baseline results in Table 4, the statistical significance is stronger (especially for univariate regressions on dealer inventory not reported) probably because of the balanced number of observations, while the economic magnitudes are similar. The two factors together account for about $42 \%$ of the unexplained total variation of credit spread changes.

Second, Table 7 reports results following the baseline procedure except using series of monthly credit spread changes (in percentage). As mentioned in Section 3.1.1, the principal component analysis in panel B shows that the first PC still accounts for $76 \%$ of the total unexplained variation of credit spread changes, similar to that in CGM but higher than in FN, both of whom also use monthly series. Panel C shows bivariate regressions on the intermediary factors for this monthly sample; the results are similar to Table 4, with stronger statistical significance, especially for dealer inventory, probably because of the large number of time series observations for each bond. The two factors together account for $20 \%$ of the unexplained total variation of credit spread changes, lower than that in the baseline analysis, not surprisingly because of a larger number of observations and higher level of variation at the monthly frequency. But the overall significant explanatory power of intermediary factors for common credit spread changes remains the same.

Finally, recall that we have constructed the intermediary distress factor $\Delta$ Distress as the first PC of both $\Delta$ Noise and $\Delta \mathrm{NLev}^{\mathrm{HKM}}{ }^{\mathrm{H}}$; this is partly for parsimony and partly for developing our model in the next section. We could have "let data speak" by regressing the credit spread residuals on these two factors separately and jointly. The results of this exercise is reported in Table 8. Similar to $\Delta$ Distress, both measures have significant pos-

\footnotetext{
${ }^{24}$ As seen from Panel A, the range of the number of bonds is 210-300, 250-450, and 170-500 in short, medium, and long term cohorts, much more homogeneous than rating-based cohorts.
} 
itive effects that monotonically decrease with bond ratings. Individually, $\Delta$ Noise accounts for $32 \%$ of the unexplained total variation of credit spread changes, higher than the $17 \%$ of $\Delta \mathrm{NLev}^{\mathrm{HKM}}$; jointly, they can explain $38 \%$. Therefore, $\Delta$ Noise and $\Delta \mathrm{NLev}^{\mathrm{HKM}}$ have overlapping but nontrivial individual explanatory power relative to each other, lending support to our construction of the intermediary distress measure as a combination of the two.

\section{An Economic Framework}

We present a simple intermediary-based setting that provides a supply-demand interpretation to our results. Supply shifts come from shocks to hedgers' endowments: because hedgers are risk-averse, shocks to their endowments initiate portfolio liquidations that increase bond supply. Demand shifts come from shocks to intermediary wealth: because intermediaries face margin constraints, balance sheet shocks affect required returns on intermediation. We show how model-based regressions with dealer inventory (a proxy for bond supply) and dealer leverage (a proxy for intermediary wealth) reproduce the qualitative patterns of our bond-level regressions. Finally, we derive further tests guided by the model.

\subsection{Setting and Equilibrium}

Assets. There are many risky assets numbered $a=1, \ldots, A$. Asset cash flows are given by $\delta$, which is normally distributed, $\delta \sim \operatorname{Normal}(\bar{\delta}, \Sigma)$. Let $p$ be the equilibrium asset price vector. There is also a riskless asset that pays 1 per unit of investment, as a normalization.

For simplicity, the risky assets are in zero net supply, i.e.,

$$
\theta_{H}+\theta_{I}=0,
$$

where $\theta_{H}$ and $\theta_{I}$ are the asset demand vectors from hedgers and intermediaries, respectively.

Hedgers. As in Kondor and Vayanos (2019), hedgers inherit a random endowment $h^{\prime} \delta$, with $h \geq 0$, and have a mean-variance objective:

$$
\begin{array}{rl}
\max _{\theta_{H}} & \mathbb{E}\left[W_{H}\right]-\frac{\alpha}{2} \operatorname{Var}\left[W_{H}\right] \\
W_{H} & :=1-w+h^{\prime} \delta+\theta_{H} \cdot\left(\delta-p-\mathbf{1}_{A}\right),
\end{array}
$$

where $W_{H}$ is ex-post hedger wealth, and $1-w$ is initial hedger wealth. The supply shocks we consider are shocks to $h$, to be described below. 
Intermediaries. Competitive, risk-neutral intermediaries maximize expected wealth, subject to a margin-type constraint:

$$
\max _{\theta_{I}} \mathbb{E}\left[w+\theta_{I} \cdot\left(\delta-p-\mathbf{1}_{A}\right)\right] \quad \text { s.t. } \quad \theta_{I} \cdot m \leq w,
$$

where $w$ is initial intermediary wealth. The constraint $\theta_{I} \cdot m \leq w$ is interpretable as a margin, or capital-adequacy, constraint, and $m$ is a vector of margin requirements or risk-weights. The demand shocks we consider are shocks to $w$, which affect the tightness of this constraint, hence intermediaries' required returns.

While the constraint $\theta_{I} \cdot m \leq w$ is sufficient to generate most of our empirical results, we also discuss other types of constraints in Appendix B.1. For example, one could argue that long and short positions should both incur margin costs, or that market prices should be in the constraint. These nuances should not dramatically alter the mechanisms we highlight.

Equilibrium. Because of the linearity of the intermediary problem, optimization implies a condition on prices

$$
p=\bar{\delta}-\mathbf{1}_{A}-\mu m
$$

where $\mu$ is the Lagrange multiplier on the margin constraint. The optimal hedger portfolio is given by the standard mean-variance optimization:

$$
\theta_{H}=(\alpha \Sigma)^{-1}\left(\bar{\delta}-p-\mathbf{1}_{A}\right)-h
$$

Using (8) in (9), and aggregating using (4), we obtain the intermediary portfolio:

$$
\theta_{I}=h-\frac{\mu}{\alpha} \Sigma^{-1} m
$$

Plugging this into the margin constraint, we have

$$
\mu=\alpha \frac{\left(m^{\prime} h-w\right)^{+}}{m^{\prime} \Sigma^{-1} m}
$$

Thus, the constraint binds (and $\mu>0$ ) if and only if $w<m^{\prime} h$, i.e., if the intermediary wealth is below the required margin from holding all the hedging demands $h$.

Corporate bond pricing. We are interested in the pricing of a subset of assets in the model. This mirrors our empirical exercise, which zooms in on the corporate bond market. Let $\mathbf{1}_{\text {bond }}$ denote the logical vector of indicators corresponding to corporate bond assets. For instance, if the first two assets are bonds and the others are not, then $\mathbf{1}_{\text {bond }}=(1,1,0,0, \ldots)^{\prime}$. 
Below, we perform comparative statics on the supply of corporate bonds and on the level of dealer liquidity-provision. The proxy for dealer liquidity-provision is intermediary wealth $w$. To proxy bond supply, write total hedging demand as $h=s \bar{h}_{\text {bond }}+\bar{h}_{\text {other }}$ for a scalar $s$ and weakly positive vectors $\bar{h}_{\text {bond }}$ and $\bar{h}_{\text {other }}$, where $\bar{h}_{\text {bond }}$ is only positive for the corporate bond assets, and the reverse for $\bar{h}_{\text {other }}$, i.e., $\bar{h}_{\text {bond }} \cdot \bar{h}_{\text {other }}=0$. We will refer to changes in $s$ a supply shock and changes in $w$ a demand shock.

Proposition 1. If the intermediary margin constraint is binding, i.e., $w<m^{\prime} h$,

$$
\begin{aligned}
\text { ("Supply Shock") } \frac{\partial p}{\partial s} & =-\left(\frac{m^{\prime} \bar{h}_{\text {bond }}}{m^{\prime} \Sigma^{-1} m}\right) \alpha m \\
\text { ("Demand Shock") } & \frac{\partial p}{\partial w}=\left(\frac{1}{m^{\prime} \Sigma^{-1} m}\right) \alpha m .
\end{aligned}
$$

Otherwise, $\frac{\partial p}{\partial s}=\frac{\partial p}{\partial w}=0$.

Proposition 1 says that increases in bond supply and decreases in intermediary demand both reduce asset prices, as one expects in a supply-demand model. Price declines occur because intermediary margin constraints "tighten" if they are faced with more bond supply or if they have lower wealth. These effects are stronger for higher-margin assets; in fact, both effects are proportional to asset margin. This is reminiscent of the monotonic pattern in empirical loadings displayed by bonds grouped by ratings or leverage. We formalize this link in the next section, where we construct model-based proxies for these supply-demand shocks and insert them into a regression framework.

One may wonder how these price effects would be modified by a more realistic margin constraint that includes asset prices, as we discuss in Appendix B.1. With prices in the constraint, the price effects of Proposition 1 would be dampened: whereas higher supply or lower demand reduces prices, falling prices then relax the intermediary margin constraint and allow for less of a risk-sharing disruption.

\subsection{Empirical Implementation}

Shock proxies. Recall in Section 2.2 our empirical pricing factors are "bond inventory" and "intermediary distress." Whereas we take as given that the second factor is closely related to $w^{-1}$, we would like to use the model to argue that the bond inventory factor is closely 
related to $s$. Define our inventory and distress factors as

$$
\begin{aligned}
\xi & :=\log \left(\theta_{I} \cdot \mathbf{1}_{\text {bond }}\right) \\
\lambda & :=w^{-1} .
\end{aligned}
$$

In equilibrium, assuming the intermediary margin constraint binds, the bond inventory measure is related to shocks $(s, w)$ as follows:

$$
\begin{aligned}
(\text { "Supply Shock") } & \frac{\partial \xi}{\partial s}=\exp (-\xi) \bar{h}_{\text {bond }} \cdot \mathbf{1}_{\text {bond }}-\left(\frac{\partial \xi}{\partial w}\right) \bar{h}_{\text {bond }} \cdot m \\
\text { ("Demand Shock") } & \frac{\partial \xi}{\partial w}=\exp (-\xi) \frac{m^{\prime} \Sigma^{-1} \mathbf{1}_{\text {bond }}}{m^{\prime} \Sigma^{-1} m} .
\end{aligned}
$$

Whatever features make inventory less sensitive to demand shocks $(w)$ make inventory more sensitive to supply shocks $(s)$, as $\partial \xi / \partial w$ enters $\partial \xi / \partial s$ negatively. ${ }^{25}$ Given that $\xi$ is affected by both $s$ and $w$ shocks, an important question is whether bond inventory increases reflect bond supply or bond demand. This is ultimately an empirical question that can be settled by examining the regression coefficient of bond prices on inventory. If this coefficient is negative, inventory must reflect bond supply to a larger extent, as high bond demand raises prices.

Bond regressions. Supposing changes to $s$ and $w$ are the only shocks, we can write

$$
\mathrm{d} p=\frac{\partial p}{\partial s} \mathrm{~d} s+\frac{\partial p}{\partial w} \mathrm{~d} w \quad \text { and } \quad \mathrm{d} \xi=\frac{\partial \xi}{\partial s} \mathrm{~d} s+\frac{\partial \xi}{\partial w} \mathrm{~d} w \quad \text { and } \quad \mathrm{d} \lambda=-(\lambda / w) \mathrm{d} w
$$

Substituting results above, we obtain an exact regression-like characterization.

Proposition 2. If $s$ and $w$ are the only non-fundamental shocks, and margin constraints bind, then

$$
\begin{aligned}
& \mathrm{d} p=\beta_{\xi} \mathrm{d} \xi+\beta_{\lambda} \mathrm{d} \lambda \\
& \beta_{\xi}:=-\exp (\xi) m^{\prime} \bar{h}_{\text {bond }}\left[\bar{h}_{\text {bond }} \cdot \mathbf{1}_{\text {bond }}-\frac{m^{\prime} \Sigma^{-1} \mathbf{1}_{\text {bond }}}{m^{\prime} \Sigma^{-1} m} \bar{h}_{\text {bond }} \cdot m\right]^{-1} \frac{\alpha m}{m^{\prime} \Sigma^{-1} m} \\
& \beta_{\lambda}:=-\frac{w}{\lambda}\left(m^{\prime} \bar{h}_{\text {bond }}\left[\bar{h}_{\text {bond }} \cdot \mathbf{1}_{\text {bond }}-\frac{m^{\prime} \Sigma^{-1} \mathbf{1}_{\text {bond }}}{m^{\prime} \Sigma^{-1} m} \bar{h}_{\text {bond }} \cdot m\right]^{-1} \frac{m^{\prime} \Sigma^{-1} \mathbf{1}_{\text {bond }}}{m^{\prime} \Sigma^{-1} m}+1\right) \frac{\alpha m}{m^{\prime} \Sigma^{-1} m} .
\end{aligned}
$$

If margin constraints are slack, then $\mathrm{d} p=0$.

\footnotetext{
${ }^{25}$ Thus, these expressions help us make sense of our empirical result that "intermediary distress" (and as well as its two ingredients "noise" and "intermediary leverage") have small negative correlations with inventory. Indeed, $\operatorname{corr}(\mathrm{d} \xi, \mathrm{d} \lambda)=-\operatorname{corr}(\mathrm{d} \xi, \mathrm{d} w)<0$.
} 
Proposition 2 allows us to relate our model directly to the data. First, the model is able to reproduce our measured pattern of regression coefficients. In Proposition 2, coefficients $\beta_{\xi}$ and $\beta_{\lambda}$ are proportional to the margin vector $m$, as alluded to earlier. Thus, the ratio of the regression betas of assets $i$ and $j$ are given by their relative margin requirements:

$$
\beta_{\xi}^{(i)} / \beta_{\xi}^{(j)}=\beta_{\lambda}^{(i)} / \beta_{\lambda}^{(j)}=m_{i} / m_{j}
$$

Lower-rated bonds and higher-leverage bonds are likely to have larger margin / capital requirements and thus should display larger loadings on both inventory and distress changes. For example, under the Basel II agreement, implemented during our sample period in many non-US jurisdictions, corporate bond holdings incur capital charges that decrease with ratings: under the so-called standardized approach, there is a $20 \%$ risk weight applied to securities rated between $\mathrm{AAA}$ to $\mathrm{AA}-; 50 \%$ for $\mathrm{A}+$ to $\mathrm{A}-; 100 \%$ for $\mathrm{BBB}+$ to $\mathrm{BB}-;$ and $150 \%$ for those below BB-. ${ }^{26}$ Relatedly, under the SEC's "net capital rule," US broker-dealers' capital requirements are tied to the riskiness of securities in their portfolios (e.g., using a VaR approach), and lower-rated corporate bonds tend to be riskier. In line with this discussion, our empirical results show inventory and dealer distress betas share a similar pattern, both rising with proxies of margin requirements. In Table 4 , we measure $\hat{\beta}_{\xi}^{\mathrm{AA}} / \hat{\beta}_{\xi}^{\mathrm{B}} \approx 10$ to 20 and $\hat{\beta}_{\lambda}^{\mathrm{AA}} / \hat{\beta}_{\lambda}^{\mathrm{B}} \approx 7$ to 15 , which are in the ballpark of the capital-requirement-implied sensitivity ratios, i.e., $m_{\mathrm{B}} / m_{\mathrm{AA}}=150 \% / 20 \%=7.5$ in Basel II. ${ }^{27}$

Second, although this model has two factors (inventory and distress), it is also consistent with a single dominant principal component, as documented in CGM and our Table 3. All non-fundamental shocks - supply and demand - alter asset prices by affecting the multiplier $\mu$ of the intermediary margin constraint (see equation (8)), thus both show up as drivers of the "single" pricing factor in an intuitive way. Here, margin $m$ represents the asset price loadings on this single factor $\mu$, analogous to bonds' eigenvector loadings on their first principal component (in the right-hand column of Table 3).

Unfortunately, the "single factor" $\mu$ is not directly measurable. Faced with this challenge,

\footnotetext{
${ }^{26}$ See page 23, paragraph 66 of https://www.bis.org/publ/bcbs128b.pdf. There is also an alternative to the standardized approach, the so-called internal ratings-based (IRB) approach, which assigns capital charges according to self-assessed default probabilities and loss-given-defaults for the underlying securities. Using the IRB approach generates qualitatively similar patterns of capital charges, because lower-rated securities have higher default probabilities and default losses.

${ }^{27}$ Although we have only included corporate bonds inventory in our regression for parsimony, this proportionality test is robust to omission of other non-corporate-bond inventories whose supply shock might be correlated with $s$. Under this model, one can show the slope coefficient for the omitted inventory variable inherits the same proportionality to vector $m$ as the included variables $\xi$ and $\lambda$. Accounting for any such omitted variable bias will modify the magnitude of $\beta_{\xi}$ and $\beta_{\lambda}$ but not their patterns. Said differently, equation (16) still holds for the biased estimates.
} 
we have instead opted to measure proxies for the shocks that drive $\mu$ (e.g., inventory as a proxy for $s$ and leverage as a proxy for $w$ ).

Developing new tests. Besides clarifying the results of Section 3, the model also allows us to design new tests. Below, we develop Predictions 1-3, which we shall take to data in Section 5.

First, although assets have many other features besides their margin requirements and capital charges, the model says that only the asset's margin matters for pricing by intermediary constraints; see equation (16). If two assets differ on some characteristic $x_{i} \neq x_{j}$, but they have the same margin $m_{i}=m_{j}$, then they will have the same sensitivities to the intermediary factors $(\xi, \lambda)$, i.e., $\beta_{\xi}^{(i)}=\beta_{\xi}^{(j)}$ and $\beta_{\lambda}^{(i)}=\beta_{\lambda}^{(j)}$. This produces the following empirical prediction.

Prediction 1. Sorting bonds by any characteristic unrelated to margin or capital requirements should not produce any pattern in sensitivities on dealer inventory or intermediary distress.

Second, the model features the following "spillover effect": when dealers take into inventory any asset carrying margin requirements, their margin constraint is tightened, and they will demand a higher premium on all other margin-carrying assets they trade. Of course, there are intuitive limitations, which are absent from our baseline model, on the extent of this spillover effect. One leading economic mechanism for such limitations is potential market segmentation across assets/dealers.

To develop a formal prediction on the extent of any spillover effects, we modify the model slightly as follows. Rather than a single margin constraint as in (7), suppose that dealers face asset-class-specific margin constraints, modeled as different constraints on non-overlapping sets of assets $\mathcal{A}_{1}$ and $\mathcal{A}_{2}$ :

$$
\sum_{a \in \mathcal{A}_{1}} \theta_{I, a} m_{a} \leq w_{1} \quad \text { and } \quad \sum_{a \in \mathcal{A}_{2}} \theta_{I, a} m_{a} \leq w_{2}
$$

There are two Lagrange multipliers, $\mu_{1}$ and $\mu_{2}$, associated with each constraint, and the pricing condition is modified to be $p=\bar{\delta}-\mathbf{1}_{A}-\operatorname{diag}(m)\left(\mu_{1} \mathbf{1}_{\mathcal{A}_{1}}+\mu_{2} \mathbf{1}_{\mathcal{A}_{2}}\right)$.

One interpretation of this modified constraint is that assets in $\mathcal{A}_{1}$ and $\mathcal{A}_{2}$ are being traded by imperfectly integrated trading desks within a bank, each with independent portfolio limits. In practice, the bank's lead portfolio manager passes on capital charges to the subsidiary trading desks, according to their individual trading positions, in the form of costs against their profit book or even trading restrictions. Under this type of model, $\mathcal{A}_{2}$ traders have 
little incentive to care about the inventory of $\mathcal{A}_{1}$ assets. Mathematically, we can show that $\mu_{1}$ is sensitive to $\mathcal{A}_{1}$ inventory, whereas $\mu_{2}$ is not (see Appendix B.2). But when the bank is in distress globally, in the sense that $w_{1}+w_{2}=w$ is reduced, all subsidiary trading desks become restricted, in the sense that both $\mu_{1}$ and $\mu_{2}$ rise. This model extension leads to the following prediction.

Prediction 2. Assets traded by corporate bond dealers (or within-dealer trading desks focused on corporate bonds) will be sensitive to bond inventory, in proportion to their margin requirements; other assets will not. All margin-carrying assets will be sensitive to aggregate intermediary distress.

Lastly, we have used the model to argue bond inventory is a good proxy for bond supply. For example, we measure $\beta_{\xi}<0$ in our regressions; see Table 4, which shows positive regression coefficients for bond yields on inventory, implying negative coefficients for bond prices. The model then implies that $\partial \xi / \partial s>0$ (see Proposition 2 and compare with equation (14)). But this line of reasoning depends on the model structure. A more direct test would be to extract plausibly exogenous supply shocks $\mathrm{d} s$ and observe how inventory $\xi$ changes.

Prediction 3. If investors liquidate some bond positions for reasons plausibly unrelated to aggregate intermediary wealth, economic conditions, or firm fundamentals, then (i) dealer bond inventory should increase; and (ii) bond prices should fall.

\section{Empirical Support to the Economic Framework}

In this section, we provide additional supporting evidence, corresponding to Predictions 1-3 above, that corroborates the key economic mechanism of dealer margin constraints. First, sorting bonds based on variables that are unlikely related to margin and capital charges does not reproduce similar regression patterns as the main findings in Section 3. Second, dealer inventory has spillover effects within the corporate credit market but not outside, while intermediary distress affects various asset classes universally. Third, dealer inventory changes are negatively associated with changes of institutional holdings of substantially downgraded bonds, lending support to the interpretation of dealer inventory factor as representing supply shocks. We further provide IV estimates for the effect of dealer inventory on credit spread changes via the supply channel. 


\subsection{Sorts on Variables Unrelated to Margin}

Our margin-based model suggests a placebo test: sorting bonds based on variables unrelated to margin should produce no pattern in price sensitivities to intermediary factors (see Prediction 1). A result of this type can be observed in Table 4, where the regression coefficients of both $\Delta$ Inventory $^{A}$ and $\Delta$ Distress are roughly similar across maturity groups, a sorting variable not strongly tied to margin requirements.

To present further evidence along this direction, we sort bonds into cohorts based on rating and trading volume, the latter of which is plausibly unrelated to a bond's margin requirements. Specifically, for each bond in each quarter, we compute the total trading volume (in dollar market value) in the last month of the quarter. Then for each quarter, we sort bonds independently into one of 15 groups based on quintiles of ratings and terciles of total trading volume. Panel A of Table 9 reports summary statistics, including the number of bonds, number of bond-quarter observations, and average total trading volume across all bonds and quarters, in each group. Within each rating category, the average total trading volume differs substantially across the tercile groups, about $\$ 2, \$ 17$, and $\$ 100$ million respectively.

Panel B of Table 9 reports time series regressions of each of the 15 residuals of quarterly credit spread changes (in percentage) on $\Delta$ Inventory $^{A}$ and $\Delta$ Distress. The magnitude and statistical significance of coefficients on both intermediary factors increase from high-rated groups to low-rated groups, consistent with the results in Section 3, but remain roughly the same across the terciles by trading volume.

\subsection{Spillover and Segmentation}

Closely-related assets are likely to be intermediated by the same dealers, or traded by the same desks within a dealer firm, and should feature a spillover effect with respect to the bond inventory factor (see Prediction 2). We provide two tests of this prediction - the first splits bond inventory into high-yield and investment-grade inventories; the second considers CDS responses to bond inventory. We expect high-yield bonds to be sensitive to investment-grade inventory (and vice versa) and CDS spreads to be sensitive to bond inventory, because these are all closely-related assets. But there is no spillover effect from corporate bond inventory to other non-corporate-credit asset classes, indicating market segmentation on this front. 


\subsubsection{High-Yield and Investment-Grade Bonds}

Similar to the aggregate inventory measure, we construct dealer inventory of high-yield $(H Y)$ and investment-grade $(I G)$ bonds separately, denoted $\Delta$ Inventor $^{H Y}$ and $\Delta$ Inventory $^{I G}$. Table 10 reports time series regressions of each of the 15 residuals of credit spread changes, on $\Delta$ Inventory $y^{I G}$ and $\Delta$ Inventory ${ }^{H Y}$ separately, as well as $\Delta$ Distress.

Both $\Delta$ Inventory ${ }^{I G}$ and $\Delta$ Inventory ${ }^{H Y}$ have explanatory power for credit spread changes of the bonds that are not in the rating categories used to compute the inventory measures, consistent with the spillover effect. As the model predicts, $\Delta$ Inventory ${ }^{H Y}$ has overall stronger effects than $\Delta$ Inventory ${ }^{I G}$ because an increase in the former tightens dealers' margin constraints more than a similar increase in the latter. Loadings on both inventory measures also feature a similar monotone effect from high-rated to low-rated bonds, as with the aggregate inventory $\Delta$ Inventory ${ }^{A}$ in Table 4.

One concern with the interpretation of these results as evidence of spillover effect is that HY and IG grade inventories may be simply correlated or driven by an unobserved common factor. Table A.8 reports correlations of the inventory measures that are inconsistent with this alternative interpretation: $\Delta$ Inventor $^{H Y}$ and $\Delta$ Inventory $^{I G}$ are negatively correlated in raw changes and near zero in percentage changes.

\subsubsection{CDS Spreads}

The second test considers CDS spreads, which are tightly-linked to corporate bonds by arbitrage, and so likely to be traded by corporate bond desks. Moreover, CDS carry capital charges, and CDS of riskier, lower-rated firms tend to have higher capital requirements. Agreements such as Basel II treat CDS as "credit risk mitigation" and, ignoring counterparty risk, tie CDS capital charges directly to the capital charges of the underlying bond (Shan, Tang, and Yan, 2016). ${ }^{28}$ Similarly, through its VaR approach, the SEC's "net capital rule" would require CDS of higher-risk firms to be held with higher capital charges.

Panel A of Table 11 reports summary statistics of our sample of quarterly CDS spread changes (in percentage). We have 1038 distinct entities, with a total of 55,744 observations at the firm-quarter level. The fraction of observations across the five rating categories remains roughly the same for different CDS maturities, about $80 \%$ with a credit rating equal to and above BBB.

\footnotetext{
${ }^{28}$ See page 46 , section 5 , paragraph 196 of https://www.bis.org/publ/bcbs128b.pdf. If the long bond position is completely hedged by a long CDS position, then the net capital charge is only related to counterparty risk. Thus, for our argument to hold, some banks trading in both bonds and CDS must not be completely hedged.
} 
We conduct quarterly time series regressions of CDS spread changes on the same set of variables as for bond yield spreads, and compute the quarterly series of residuals. For each quarter and each maturity, we sort firms into one of the five groups of credit rating and take an average of the residuals within each group and in each quarter. Panel B of Table 11 reports the principal component analysis of the CDS spread change residuals. Similar to the baseline evidence in Table 3, the first PC accounts for over $80 \%$ of the common variation in CDS spread changes. Panel C reports regressions of these residuals on dealers' bond inventory and intermediary distress. The patterns of regression coefficients mirror those for bonds themselves, i.e., positive and monotonically decreasing with bond rating. The total explanatory power is lower than the $48 \%$ in the bond evidence, but still reaches $37 \%{ }^{29}$

\subsubsection{Non-Corporate-Credit Asset Classes}

These spillover effects may be limited by the presence of some market segmentation. To investigate this, we perform a similar analysis on a host of non-corporate-credit asset classes, which are less likely to be traded by corporate bond dealers or corporate credit trading desks within a dealer firm. Specifically, we regress quarterly changes of yield spreads (relative to Treasury) of agency MBS, CMBS, ABS, and monthly S\&P 500 index option returns on the time series variables to extract the residuals. We then run time series regressions of these residuals on $\Delta$ Inventory $^{A}$ and $\Delta$ Distress. According to Prediction 2, these assets should be insensitive to bond inventory changes, but should still respond to aggregate intermediary distress. Table 12 shows results consistent with this prediction.

\subsection{Bond-Level Evidence of Supply Shocks and IV Regressions}

In this section, by delving into bond-level dealer inventories and institutional holdings, we provide evidence that the change of dealer inventory is driven by the supply of bonds from investors who experience liquidity shocks. Based on such micro-level evidence, we then construct instruments for the dealer inventory factor at the aggregate level and conduct IV analysis of the effect of dealer inventory on credit spread changes.

A word of caution: bond downgrades clearly contain information about firm fundamentals and economic conditions, so we cannot argue that investor sell-offs are truly exogenous

\footnotetext{
${ }^{29}$ One may concern that the sensitivity of CDS spreads to bond inventory reflects some latent unobservable common credit risk factor. We provide two results to mitigate this concern. First, time series credit risk controls are included in regressions to obtain CDS spread change residuals. Second, results remain the same using the sample of CDS for which the underlying entities are not matched to the firms in the sample of TRACE transactions of corporate bonds used to construct the dealer inventory measure.
} 
"supply shocks" (as in Prediction 3). But recall that when constructing the residuals of credit spread changes we have controlled for firm- and market-level structural factors. Moreover, severe downgrades from IG rating to HY rating, also called "fallen angels," are more likely to serve as pure supply shocks, thanks to regulatory constraints imposed on financial institutions. Our later IV analysis uses "fallen angels" (controlling normal downgrades) together with the insured losses due to natural disasters to instrument the supply shock.

\subsubsection{Bond-Level Evidence of Supply Shocks}

Our bond-level analysis makes use of rating downgrades of bonds that can lead to large sell-offs from institutional investors. We provide evidence that a significant amount of such sell-offs are absorbed into dealers' balance sheet as inventories.

Holding Changes in Downgrades and Fallen Angels: Raw Data We proceed with the data as follows. From Mergent FISD, we obtain the dates and reasons of all bonds' historical rating changes. From TRACE, we compute the total inventory change of all dealers for each bond $i$ in each quarter $t$, denoted as $\Delta$ Inventor $_{i, t}$. From eMAXX, we compute the change of total holdings for each bond $i$ and in each quarter $t$, denoted as $\Delta H_{\text {olding }}, t$, by each of three groups of institutional investors - insurance companies, mutual funds, and pension funds. We identify observations of $\Delta$ Inventory $_{i, t}$ and $\Delta$ Holding $_{i, t}$ as "downgrade" observations if bond $i$ is downgraded from IG rating to IG or HY rating in quarter $t$ and as "no rating change" observations if the credit rating remains unchanged. Among "downgrade" observations, we further identify "fallen angels" that have been downgraded from IG rating to HY rating (Ambrose, Cai, and Helwege (2008), Ellul, Jotikasthira, and Lundblad (2011)) and "downgrade (IG)" observations with bonds downgraded from IG rating to a lower IG rating. Our analysis relies on the sell-offs induced by bond downgrading, so we exclude "upgrade" observations. ${ }^{30}$ We also exclude observations with bonds downgraded from HY rating to a lower HY rating, as the different initial rating category makes it hard to compare with "fallen angel" observations.

Table 13 reports the average quarterly change of holdings by insurance companies, mutual funds, and pension funds, in panels A, B, and C, respectively, and the average quarterly change of dealers' inventories in panel D. In particular, for each of these four groups of investors, we report the average of $\Delta$ Holding $_{i, t}$ or $\Delta$ Inventory $_{i, t}$ across "downgrade (IG)", "fallen angels", and "no rating change" observations. Both the number of observations and

\footnotetext{
${ }^{30}$ Including "upgrade" observations as a comparison group leads to stronger results in quantifying the sell-offs of downgraded bonds by institutional investors, not surprisingly.
} 
average holdings change (in \$million) are reported. We also include the average of changes in quarter $t+1$, i.e., one quarter following the rating change, because it may take time for investors to adjust their positions. To put the magnitudes in context, we report the average level of institutional holding Holding $_{i, t-1}$ and dealer inventory Inventory $y_{i, t-1}$ as of quarter $t-1$ in the last row of each panel.

For the set of "downgrade (IG)" bonds, both at the quarter of downgrade $(t)$ and the following quarter $(t+1)$, insurance companies decreased their holdings by about $\$ 0.92-1.01$ million, while mutual funds and pension funds increased their holdings by about $\$ 0.29-0.38$ million at quarter $t$ but sold about $\$ 0.16-0.25$ million at quarter $t+1$. Similar patterns are found for "fallen angels." In particular, insurance companies sold off "fallen angels" in both quarters, about $\$ 1.27-1.35$ million, while mutual funds and pension funds bought about $\$ 0.12-0.20$ million at quarter $t$ and sold about $\$ 0.24-0.47$ million at quarter $t+1$. In other words, the sell-offs by insurance companies are larger for "fallen angels" than for "downgrade (IG)" bonds, whereas purchases by mutual funds and pension funds are smaller for "fallen angels" than "downgrade (IG)" bonds. This is consistent with insurance companies being forced to sell downgraded bonds, especially "fallen angels" due to regulatory constraints, and mutual funds and pension funds purchasing these bonds to take advantage of "fire-sale" discounts (Cai, Han, Li, and Li (2019), Anand, Jotikasthira, and Venkataraman (2018)).

Importantly, panel D of Table 13 shows that dealers buy a similar amount of "downgrade (IG)" bonds in quarter $t$ to mutual funds and pension funds, about $\$ 0.34$ million, but a much larger amount of "fallen angels", about $\$ 1.31$ million. Dealers also buy "downgrade (IG)" bonds and sell "fallen angels" in quarter $t+1$, but in small amounts. Compared with the level of inventory as of quarter $t-1$, dealers' purchase amount of "fallen angles" is strikingly large, an increase of about $78 \%$, substantially larger than those of mutual funds and pension funds that are below $1 \%$.

Holding Changes in Downgrades and Fallen Angels: Regression Next, we conduct regression analysis - which allows us to control bond characteristics - to formally test the relation between institutional investors' sell-offs and dealers' inventory changes. The first three columns of Table 14 report results based on the following regression:

$$
\begin{aligned}
& \Delta \text { Holding }_{i, t+\tau} \\
= & \text { Intercept }+\beta_{1} \times \text { Fallen }_{i, t}+\beta_{2} \times \text { Downgrade }_{i, t}+\beta_{3} \times \log \left(\mathrm{Amt}_{i, t+\tau}\right)+\beta_{4} \times \log \left(\operatorname{Size}_{i}\right) \\
& +\beta_{5} \times \text { Age }_{i, t+\tau}+\beta_{6} \times \text { Time-to-Mature }_{i, t+\tau}+\sum_{t} \mathrm{FE}_{t}+\varepsilon_{i, t+\tau},
\end{aligned}
$$


where $\tau=0$ for the change in quarter $t$ (reported in panel A) and $\tau=1$ for the change in

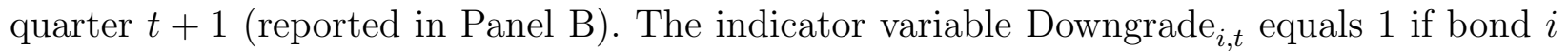
is downgraded in quarter $t$ and 0 otherwise, whereas Fallen ${ }_{i, t}$ equals 1 if bond $i$ is a "fallen angel" in quarter $t$ and 0 otherwise.

The sample includes "downgrade (IG)", "fallen angels", and "no rating change" observations. Thus, the coefficient on Downgrade ${ }_{i, t}$ captures the $(t+\tau)$ change of institutional investors' holdings of a average downgraded bond in quarter $t$, relative to that of a bond that has no rating change contemporaneously. Similarly, the coefficient on Fallen ${ }_{i, t}$ captures the $(t+\tau)$ change of institutional investors' holdings of a bond downgraded from IG rating to HY rating in quarter $t$, relative to the average of those that have no rating change and that are downgraded from IG rating to IG rating contemporaneously. We control for various bond characteristics including the log of outstanding balance $\left(\log \left(\mathrm{Amt}_{i, t+\tau}\right)\right)$, the log of issue size $\left(\log \left(\operatorname{Size}_{i}\right)\right)$, bond age $\left(\right.$ Age $\left._{i, t+\tau}\right)$, and time-to-maturity (Time-to-Mature $\left.{ }_{i, t+\tau}\right)$, and also include time fixed effects. Panel regressions of changes in dealers' inventories $\Delta$ Inventor $_{i, t+\tau}$, similar to (17) are reported in the last column.

Consistent with summary statistics in Table 13, we observe that insurance companies decrease their holdings of downgraded bonds in both quarters, about $\$ 0.48-0.80$ million, relative to the bonds with no rating changes. Mutual funds and pension funds seem to take some of the downgraded bonds sold by insurance companies in quarter $t$, about $\$ 0.51$ and $\$ 0.36$ million respectively, but no detectable patterns in the following quarter. Insurance companies sell "fallen angels" even more aggressively, about $\$ 0.67$ million in quarter $t$ and $\$ 0.33$ million in quarter $t+1$, relative to average bonds that experience no rating changes and that are downgraded from IG rating to IG rating. Mutual funds and pension funds do not conduct significant purchases of "fallen angles". In contrast, dealers' inventories of "fallen angels" increase substantially in quarter $t$ (about $\$ 1.61$ million) and then decrease somewhat in quarter $t+1$ (about $\$ 0.45$ million). That is, dealers first take inventories of "fallen angels" in providing liquidity to insurance companies, and then unwind (part of) these inventories at a later time, consistent with standard inventory control behavior (Ho and Stoll, 1981). Interestingly, dealers' inventories of average downgraded bonds do not seem to be significantly different from those with no rating change.

In sum, insurance companies dump a large amount of "fallen angels", and dealers take them into their inventories. Taking as a premise that insurance companies face constraints due to regulations for holding low-rated bonds (Ellul, Jotikasthira, and Lundblad, 2011), we interpret downgrade-induced sell-offs by insurance companies as a supply shock to increase dealers' inventories, independent of their balance sheet condition (wealth or leverage). In the 
following, we construct an IV for the dealer inventory factor based on institutional investors' liquidations of "fallen angels."

\subsubsection{Regressions}

To construct a time series IV for the dealer inventory factor $\Delta$ Inventor $y_{t}^{A}$, we aggregate the changes of institutional holdings of downgraded bonds in each quarter. In particular, we use the sell-offs of "fallen angels" $\Delta$ Holding $F_{t}^{F A}$ as the IV and the sell-offs of all downgraded bonds $\Delta$ Holding $_{t}^{D}$ as a control. Using $\Delta H$ Holding $g_{t}^{D}$ as a control (partially) takes care of the confound that downgrading contains information on the fundamental value of bonds, which then leads to both sell-offs and price effects. In addition, recall that the sell-offs of "fallen angels" come mainly from insurance companies that face clear-cut regulatory constraints, so $\Delta H o l d i n g_{t}^{F A}$ is likely a purer proxy for bond supply shocks than $\Delta H o l d i n g_{t}^{D}$. Still, we include all types of institutional investors when computing $\Delta H_{\text {olding }}^{F A}$ and $\Delta H_{\text {olding }}^{D}$, not only insurance companies, to capture the net selling to dealers, given that mutual funds and pension funds seem to take some amount of downgraded bonds sold by insurance companies. ${ }^{31}$ We scale $\Delta$ Holding $_{t}^{F A}$ and $\Delta$ Holding $_{t}^{D}$ by their respective levels of holdings as of $t-1$, corresponding to our construction of $\Delta$ Inventor $y_{t}^{A}$ as a percentage change.

To complement the analysis using $\Delta H_{\text {olding }}^{F A}$, we also construct a second IV using insured losses due to natural disasters. In particular, we obtain from the Insurance Information Institute an annual series of realized industry-wide losses from catastrophes, capturing the total net insurance payment for personal and commercial property lines of insurance. ${ }^{32}$ A linear time series model is fitted to the logarithm of this annual series with the residuals as the payout shocks. As catastrophes mostly happen in the third quarter of the year (e.g., hurricanes), we assign each year's payout shock to the third quarter and zero to other quarters. The resulting quarterly series is denoted by InsuredLoss ${ }_{t}$.

The rationale for using InsuredLoss ${ }_{t}$ as an IV is that unexpected insured losses are likely to induce sell-offs of corporate bonds by insurance companies to make payments. The advantage of InsuredLoss to $\Delta$ Holding $_{t}^{F A}$ as an IV is its clear-cut exogeneity to bond-value shocks.

\footnotetext{
${ }^{31}$ Of course, this will overestimate (underestimate) the net selling amount to dealers if other investors like hedge funds also buy (sell) some amount of.

${ }^{32}$ The included catastrophes, following definitions of the Property Claim Services division of Verisk Analytics, are those that caused insured property losses of $\$ 25$ million or more in 1997 dollars and affected a significant number of policyholders and insurers, excluding losses covered by the federally administered National Flood Insurance Program. The types of catastrophes include, for example, wildfires, heat waves, droughts, tropical cyclones, severe thunderstorms, winter storms, cold waves, floods, and earthquakes. See https://www.iii.org/fact-statistic/facts-statistics-us-catastrophes\#Loss\% 20Events $\% 20$ in $\% 20$ the $\% 20$ U.S. , $\% 201980-2018$ for detailed information about natural catastrophes and https://www.iii.org/table-archive/20922 for our data series.
} 
The disadvantage is that selling of corporate bonds may only be one of the strategies for insurance companies to collect payment money, which, for example, can be achieved by selling other fixed-income securities like Treasuries or agency MBS, or by withdrawing their cash lent out in short-term funding markets. Moreover, insured looses are only available at the annual level, and they are only shocks to P\&C insurers, leaving life insurers unaffected. These issues may make InsuredLoss $t$ a statistically-weak IV, which needs to be addressed using proper econometric procedures.

Table 15 reports first-stage regressions of $\Delta$ Inventory $^{A}$ on $\Delta$ Holding $_{t}^{F A}$ and InsuredLoss ${ }_{t}$, separately in the first two columns and jointly in the third column. As mentioned above, we include $\Delta$ Holding $g_{t}^{D}$ as a control, in addition to the intermediary distress factor and six time series variables also used in the baseline bond-level regressions (1). All variables are scaled to have zero mean and unit variance except the six time series variable from (1). We observe that a one-standard-deviation decrease in institutional holdings of "fallen angels" is associated with about a 0.37 standard deviation increase in dealer inventory, indicating the relevance of $\Delta$ Holding $_{t}^{F A}$ for $\Delta$ Inventory $^{A}$. A one-standard-deviation increase of InsuredLoss $_{t}$ is associated with a 0.07 standard derivation increase in dealer inventory, but as expected, the statistical significance is weak.

Table 16 reports second-stage regressions of quarterly residuals of credit spread changes

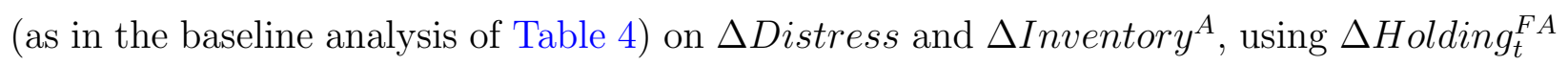
as an inventory IV in Panel A, using InsuredLoss $t$ as an inventory IV in Panel B, and using both as IVs in Panel C. The last two rows in each panel reports the test statistic for weak instruments by Montiel-Olea and Pflueger (2013) (MP) and associated critical values (Pflueger and Wang, 2015), which allow for conditionally-heteroskedastic and seriallycorrelated errors. ${ }^{33}$

From Panel A of Table 16, the value of the MP-statistic is larger than the critical value, so the null hypothesis that $\Delta H_{\text {olding }}^{F A}$ is a weak investment for $\Delta$ Inventor $y_{t}^{A}$ is rejected at a significance level of $10 \%$ with a worst-case bias greater than $20 \%$ of the OLS bias. The regression coefficients and t-statistics reported in parentheses show that the dealer inventory factor instrumented by institutional holdings of "fallen angels" is highly significant in affecting credit spread changes positively, consistent with the baseline results in Section 3.

The MP test reported in Panel B of Table 16 is below the critical value, so we cannot reject the null hypothesis that InsuredLoss ${ }_{t}$ is a weak instrument. Therefore, for testing the significance of the instrumented $\Delta$ Inventor $y_{t}^{A}$, we choose to report p-values of the Anderson

\footnotetext{
${ }^{33}$ The widely used Cragg and Donald (1993) test and associated critical values by Stock and Yogo (2005) are only valid for conditionally-homoskedastic and serially-uncorrelated errors.
} 
and Rubin (1949) Wald-test in the left bracket and p-values of the Stock and Wright (2000) Sstatistic in the right bracket, both of which are weak-instrument robust. The dealer inventory factor instrumented by InsuredLoss $t$ affects credit spread changes significantly at the $10 \%$ level mainly for groups of short to medium maturities and low ratings based on the Anderson and Rubin (1949) Wald-test, but only at the $15 \%$ significance level based on the Stock and Wright (2000) S-test. The value of the MP-statistic in Panel C is larger than the critical value, so weak instrument is less of a concern when we use both $\Delta H_{\text {olding }}^{F A}$ and InsuredLoss ${ }_{t}$ as IVs. The regression coefficients and t-statistics further confirm the positive effect of dealer inventory on credit spread changes.

We note that the coefficients in IV regressions, especially on $\Delta$ Inventor $y_{t}^{A}$, are significantly larger than those in the baseline regressions of Table $4 .{ }^{34}$ One potential reason is that dealer inventory changes could be driven by (unobserved) demand shocks (e.g., increased optimism of dealers regarding bond fundamentals, higher risk taking of dealers' bond trading desks, or looser regulatory requirements). ${ }^{35}$ Such a demand shock leads to higher dealer inventory but lower credit spreads, which biases against the positive supply-driven co-movement of dealer inventory and credit spreads. Using the two IVs, which we claim are purely about supply, can purge our price-inventory sensitivity of demand effects.

\section{Conclusion}

It has been almost two decades since CGM raised one of the canonical puzzles in asset pricing of credit risk, i.e., there is a large common variation in credit spread changes beyond structural factors. In this paper, we build on recent developments in intermediary asset pricing (He and Krishnamurthy, 2013; He, Kelly, and Manela, 2017) and demonstrate the importance of intermediary constraints to explain this canonical puzzle.

In particular, we show that two intermediary-based factors, an intermediary distress measure that captures financial constraints of the whole intermediary sector and a dealer inventory measure that captures inventory held by dealers specializing in corporate bonds, explain about $50 \%$ of the puzzling common variation documented in CGM. A simple eco-

\footnotetext{
${ }^{34}$ Another difference between the IV regressions and baseline regressions (2) is that the former includes additional time series controls. These controls are not included in the baseline regressions because they have been controlled for in the bond-level regressions (1) used to construct the residuals. We include them in IV regressions to make sure the IV analysis is robust to them, which, however, is not the reason for the larger regression coefficients on $\Delta$ Inventory $y_{t}^{A}$.

${ }^{35}$ The demand shock could theoretically also be a distress-type shock, which affects bond inventory, as we show in our model. However, we view this possibility as unlikely, given we find the IV-OLS discrepancy in a multivariate setting, with our Distress measure included.
} 
nomic framework in which intermediaries face margin constraints and absorb assets sales from customers delivers the robust empirical pattern that both intermediary distress and bond inventory factors are associated with credit spread changes, and these effects are monotone in bond ratings.

We construct the aggregate corporate bond inventory for the broker-dealer sector, which can facilitate future research. We also augment this inventory measure with data on corporate bond holdings by other institutional investors (insurance companies, mutual funds, and pension funds). An important component of dealers' inventory change is tied to institutional investors' sales of (severely) downgraded bonds, which we interpret as a supply shock.

Following the spirit of CGM, we have mainly focused on using non-bond-return-based factors to explain the time series variations of credit spreads. Given that credit spread changes are inherently tied to bond returns, a natural question is whether our non-bondreturn-based intermediary factors are related to bond-return-based factors proposed in the literature that focus on explaining cross-sectional bond returns. As an exploratory analysis, Table 17 presents regressions of four bond-return factors of Bai, Bali, and Wen (2019) on our two intermediary factors. After orthogonalizing all factors - their return-based factors and our non-return-based factors - to time series variables in the individual bond regressions (1), we find that intermediary distress comoves with all return-based factors significantly, but not dealer inventory. This result suggests that intermediary distress provides a potential fundamental-based explanation for these return-based factors of Bai, Bali, and Wen (2019), while we have yet to find some other return-based-factors to proxy for dealer inventory. This can be a fertile future research direction. 


\section{Figure 1: Quarterly Time Series of Intermediary Factors and CGM PC1}
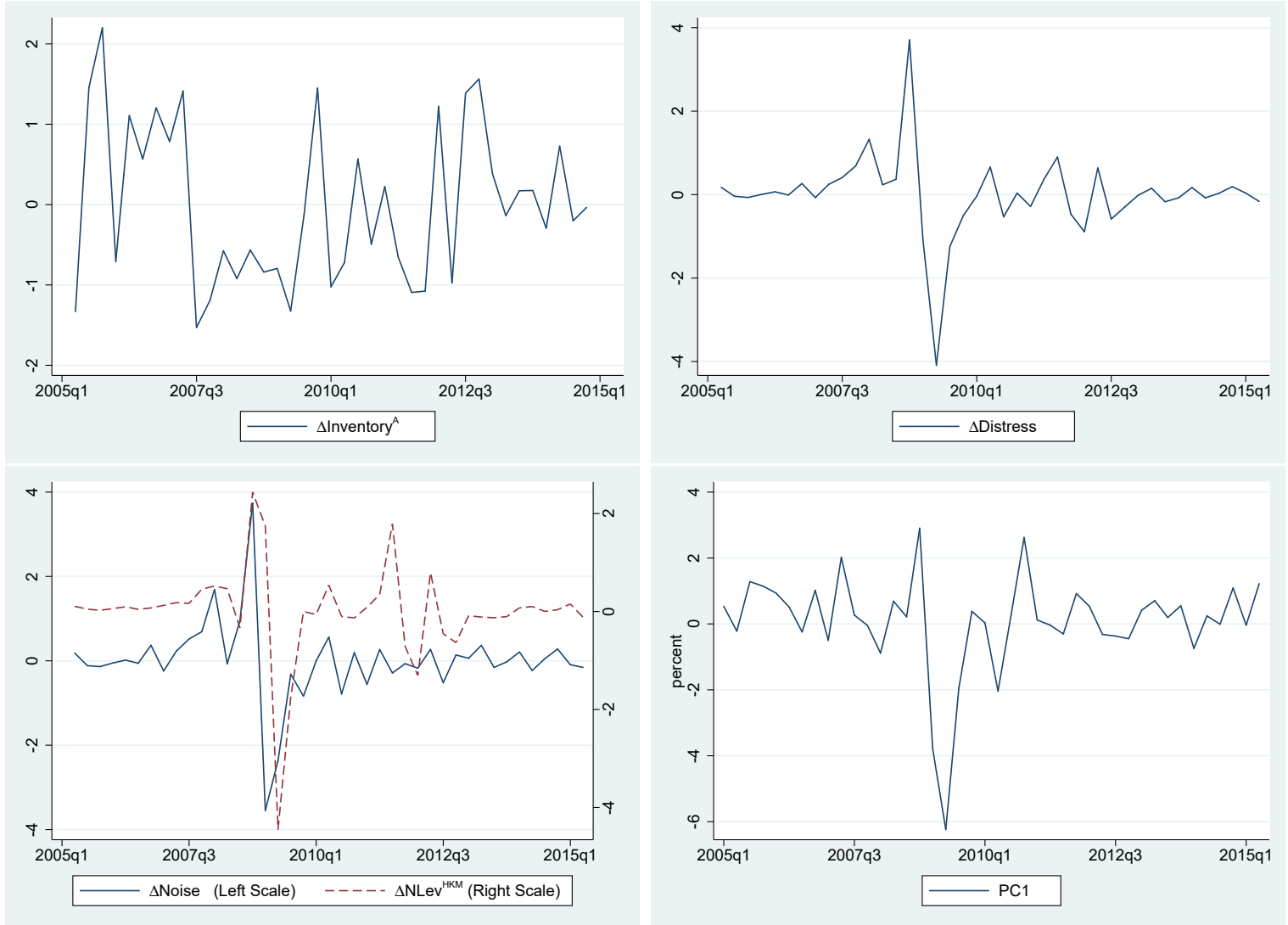

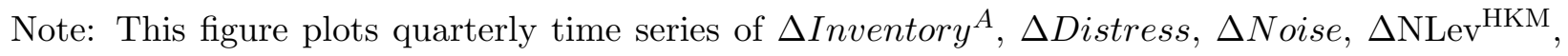
and the first principal component of regression residuals of credit spread changes on structure factors (CGM PC1) as reported in Table 3. The sample period is from 2005:Q1 through 2015:Q2. The four intermediary variables are standardized to zero mean and unit standard deviation, and the CGM PC1 is based on 90-day change of credit spreads in percent. 
Figure 2: Summary of Amount of Institutional Holdings and Dealer Inventories

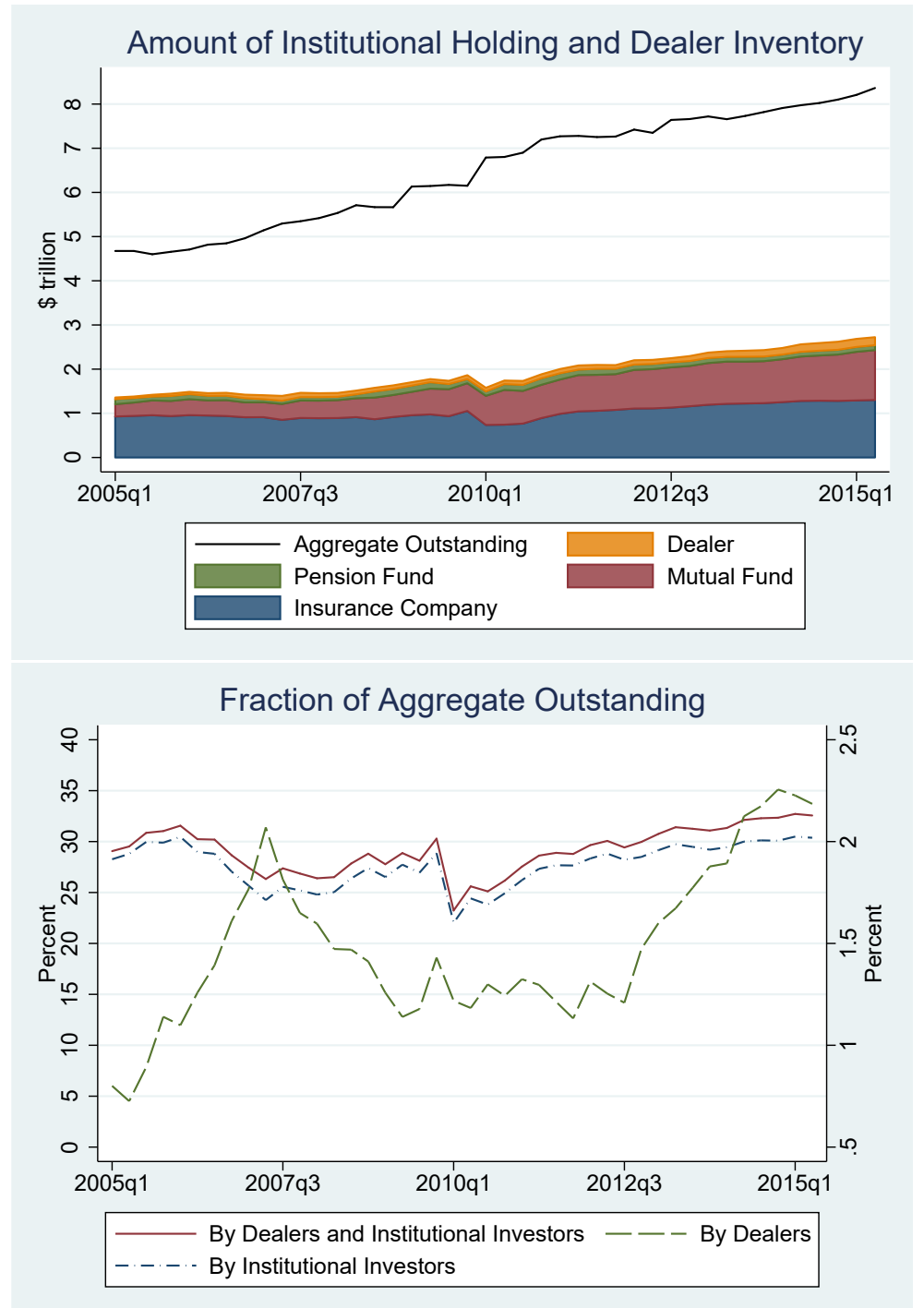

Note: The top panel plots quarterly time series of the holding amount by institutional investors (including mutual funds, pension funds, and insurance companies) based on eMAXX data and by dealers based on TRACE data, as well as the aggregate outstanding balance of U.S. corporate debt securities ("L.208 Debt Securities" series) based on the "Financial Accounts of the United States" (Z.1) data release by the Federal Reserve, in \$trillions of principal value. The bottom panel plots quarterly time series of the fraction of U.S. corporate debt securities held by institutional investors, by dealers, and by both, respectively, in percent. The sample period is from 2005:Q1 through 2015:Q2. 


\section{Table 1: Summary of the Credit Spread Sample}

\begin{tabular}{|c|c|c|c|c|c|}
\hline \multicolumn{6}{|c|}{ All Bonds } \\
\hline Number of bonds & 2,584 & & & & \\
\hline Number of firms & 653 & & & & \\
\hline Number of bond-quarters & 55,398 & & & & \\
\hline & mean & std & $\mathrm{p} 25$ & $\mathrm{p} 50$ & p75 \\
\hline Yield spread & 2.51 & 2.69 & 0.95 & 1.60 & 3.12 \\
\hline Coupon & 6.32 & 1.59 & 5.38 & 6.30 & 7.25 \\
\hline Time-to-Maturity & 9.78 & 8.07 & 4.19 & 6.80 & 11.84 \\
\hline Age & 5.12 & 4.32 & 2.14 & 3.86 & 6.67 \\
\hline Issuance & 550.50 & 471.97 & 250.00 & 400.00 & 650.00 \\
\hline Rating & 9.25 & 3.43 & 7.00 & 9.00 & 11.00 \\
\hline \multicolumn{6}{|c|}{ Investment Grade Bonds } \\
\hline Number of bonds & 1,980 & & & & \\
\hline Number of firms & 383 & & & & \\
\hline Number of bond-quarters & 40,828 & & & & \\
\hline & mean & std & p25 & $\mathrm{p} 50$ & p75 \\
\hline Yield spread & 1.52 & 1.17 & 0.81 & 1.22 & 1.85 \\
\hline Coupon & 5.87 & 1.42 & 5.00 & 5.90 & 6.75 \\
\hline Time-to-Maturity & 10.85 & 8.76 & 4.21 & 7.38 & 17.56 \\
\hline Age & 5.34 & 4.46 & 2.21 & 4.01 & 7.06 \\
\hline Issuance & 605.62 & 505.64 & 300.00 & 500.00 & 750.00 \\
\hline Rating & 7.58 & 1.90 & 6.00 & 8.00 & 9.00 \\
\hline \multicolumn{6}{|c|}{ High Yield Bonds } \\
\hline Number of bonds & 900 & & & & \\
\hline Number of firms & 373 & & & & \\
\hline \multirow[t]{2}{*}{ Number of bond-quarters } & 14,570 & & & & \\
\hline & mean & std & $\mathrm{p} 25$ & $\mathrm{p} 50$ & $\mathrm{p} 75$ \\
\hline Yield spread & 5.27 & 3.65 & 3.15 & 4.46 & 6.12 \\
\hline Coupon & 7.60 & 1.33 & 6.75 & 7.50 & 8.25 \\
\hline Time-to-Maturity & 6.78 & 4.50 & 4.14 & 5.92 & 7.80 \\
\hline Age & 4.53 & 3.87 & 1.97 & 3.49 & 5.69 \\
\hline Issuance & 396.04 & 313.28 & 200.00 & 300.00 & 500.00 \\
\hline Rating & 13.96 & 2.15 & 12.00 & 14.00 & 16.00 \\
\hline
\end{tabular}

Note: This table reports bond characteristics for our baseline sample of credit spreads. We report the mean, standard deviation (sd), median (p50), 25th percentile (p25), and 75th percentile (p75) for the whole sample, investment grade subsample, and high yield subsample. The total number of bonds is smaller than the sum of the number of bonds in the investment grade and high yield subsamples because rating change make some bonds of investment grade in one part of the sample period but of high yield in the other part. Credit spread (in percentage) is the difference between the annualized yield-to-maturity of a corporate bond and a Treasury with the same maturity calculated with linear interpolations whenever necessary. Coupon is the coupon rate in percent. Time-to-maturity is in units of years. Age is the number of years since issuance. Issuance size is in \$millions of face value. Rating is the Moody's credit rating of a bond coded numerically so that a higher number means lower rating, e.g., $\mathrm{Aaa}=1$ and $\mathrm{C}=21$. The overall sample period is 2005:Q1 - 2015:Q2 
Table 2: Correlations of Empirical Measures

\begin{tabular}{lllllll}
\hline & $\Delta$ Inventory $^{A}$ & $\Delta$ Distress & $\Delta$ Noise & $\Delta$ NLev $^{\mathrm{HKM}}$ & $\Delta V I X$ & $\Delta \mathrm{ILiq}$ \\
\cline { 2 - 7 } Inventory $^{A}$ & 1.000 & & & & & \\
$\Delta$ Distress & -0.116 & 1.000 & & & & \\
$\Delta$ Noise & -0.094 & $0.833^{* * *}$ & 1.000 & & & \\
$\Delta$ NLev $^{\text {HKM }}$ & -0.099 & $0.833^{* * *}$ & $0.388^{* *}$ & 1.000 & & \\
$\Delta$ VIX & -0.094 & $0.357^{* * *}$ & 0.167 & $0.427^{* * *}$ & 1.000 & \\
$\Delta$ ILiq & -0.106 & 0.228 & 0.192 & 0.188 & $0.381^{* *}$ & 1.000 \\
\hline
\end{tabular}

Note: This table reports correlations of quarterly time series of $\Delta$ Inventory $^{A}, \Delta$ Distress, $\Delta \mathrm{NLev}^{\mathrm{HKM}}$, $\Delta$ Noise, $\Delta V I X$, and $\Delta$ ILiq. The sample period is from 2005:Q1 through 2015:Q2. Significance levels are represented by $* \mathrm{p}<0.1,{ }^{* *} \mathrm{p}<0.05$, and ${ }^{* * *} \mathrm{p}<0.01$ with $\mathrm{p}$ as the $\mathrm{p}$-value. 


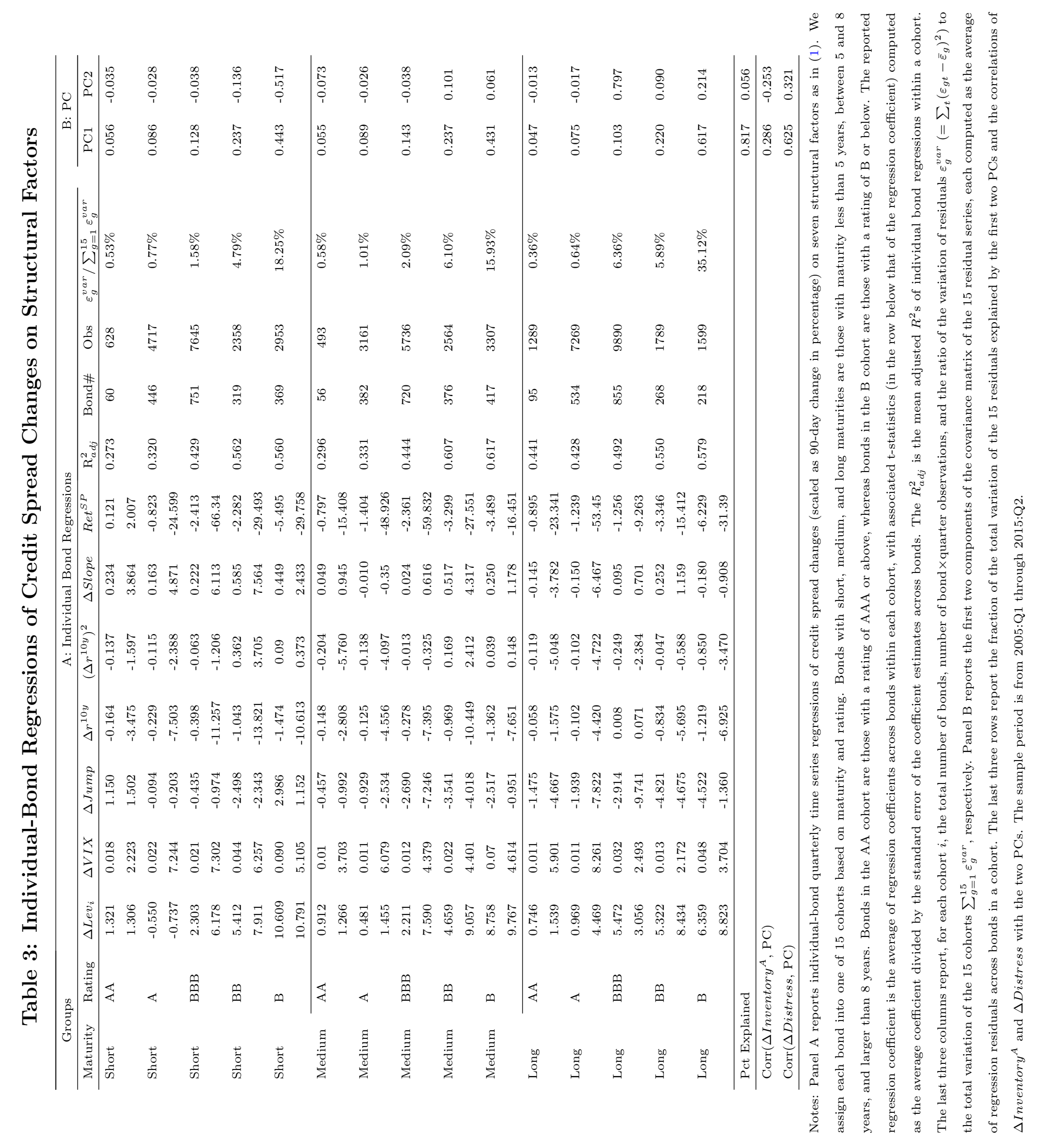


Table 4: Regressions of Credit Spread Change Residuals on Intermediary Factors

\begin{tabular}{|c|c|c|c|c|c|c|c|c|c|}
\hline \multicolumn{2}{|c|}{ Groups } & \multicolumn{2}{|c|}{ A: $\Delta$ Inventory ${ }^{A}$} & \multicolumn{2}{|c|}{ B: $\Delta$ Distress } & \multicolumn{4}{|c|}{ C: $\Delta$ Inventory $^{A}+\Delta$ Distress } \\
\hline Maturity & Rating & $\Delta$ Inventory $^{A}$ & $\mathrm{R}_{a d j}^{2}$ & $\Delta$ Distress & $\mathrm{R}_{a d j}^{2}$ & $\Delta$ Inventory $^{A}$ & $\Delta$ Distress & $\mathrm{R}_{a d j}^{2}$ & FVE \\
\hline Short & AA & $\begin{array}{c}0.023 \\
(1.275)\end{array}$ & 0.040 & $\begin{array}{l}0.039^{*} \\
(1.946)\end{array}$ & 0.114 & $\begin{array}{c}0.026 \\
(1.341)\end{array}$ & $\begin{array}{c}0.053^{* * *} \\
(3.302)\end{array}$ & 0.212 & 0.378 \\
\hline Short & $\mathrm{A}$ & $\begin{array}{c}0.019 \\
(0.916)\end{array}$ & 0.018 & $\begin{array}{c}0.059^{* * *} \\
(2.669)\end{array}$ & 0.167 & $\begin{array}{l}0.033^{*} \\
(1.935)\end{array}$ & $\begin{array}{c}0.078^{* * *} \\
(4.688)\end{array}$ & 0.297 & \\
\hline Short & $\mathrm{BBB}$ & $\begin{array}{c}0.025 \\
(0.944)\end{array}$ & 0.015 & $\begin{array}{c}0.105^{* * *} \\
(3.209)\end{array}$ & 0.260 & $\begin{array}{c}0.046^{* *} \\
(2.204)\end{array}$ & $\begin{array}{c}0.133^{* * *} \\
(4.825)\end{array}$ & 0.411 & \\
\hline Short & $\mathrm{BB}$ & $\begin{array}{c}0.062 \\
(0.860)\end{array}$ & 0.017 & $\begin{array}{c}0.161^{* * *} \\
(3.002)\end{array}$ & 0.153 & $\begin{array}{c}0.095^{* *} \\
(2.143)\end{array}$ & $\begin{array}{c}0.203^{* * *} \\
(5.384)\end{array}$ & 0.337 & \\
\hline Short & $\mathrm{B}$ & $\begin{array}{c}0.198^{* *} \\
(2.269)\end{array}$ & 0.078 & $\begin{array}{l}0.298^{*} \\
(1.929)\end{array}$ & 0.170 & $\begin{array}{c}0.294^{* * *} \\
(3.909)\end{array}$ & $\begin{array}{c}0.370^{* * *} \\
(3.740)\end{array}$ & 0.383 & \\
\hline Medium & AA & $\begin{array}{c}0.022 \\
(1.105)\end{array}$ & 0.032 & $\begin{array}{c}0.046^{* * *} \\
(3.436)\end{array}$ & 0.130 & $\begin{array}{c}0.011 \\
(0.591)\end{array}$ & $\begin{array}{c}0.048^{* * *} \\
(3.956)\end{array}$ & 0.140 & 0.550 \\
\hline Medium & $\mathrm{A}$ & $\begin{array}{l}0.041^{*} \\
(1.733)\end{array}$ & 0.060 & $\begin{array}{c}0.087^{* * *} \\
(2.588)\end{array}$ & 0.264 & $\begin{array}{c}0.048^{* *} \\
(2.132)\end{array}$ & $\begin{array}{c}0.093^{* * *} \\
(3.661)\end{array}$ & 0.342 & \\
\hline Medium & $\mathrm{BBB}$ & $\begin{array}{c}0.064^{* *} \\
(2.097)\end{array}$ & 0.071 & $\begin{array}{c}0.137^{* * *} \\
(2.730)\end{array}$ & 0.317 & $\begin{array}{c}0.075^{* *} \\
(2.543)\end{array}$ & $\begin{array}{c}0.146^{* * *} \\
(4.030)\end{array}$ & 0.410 & \\
\hline Medium & $\mathrm{BB}$ & $\begin{array}{l}0.130^{*} \\
(1.902)\end{array}$ & 0.098 & $\begin{array}{c}0.235^{* * *} \\
(4.230)\end{array}$ & 0.321 & $\begin{array}{c}0.129^{* * *} \\
(3.050)\end{array}$ & $\begin{array}{c}0.251^{* * *} \\
(5.934)\end{array}$ & 0.414 & \\
\hline Medium & $\mathrm{B}$ & $\begin{array}{c}0.172^{* *} \\
(2.041)\end{array}$ & 0.067 & $\begin{array}{c}0.465^{* * *} \\
(3.444)\end{array}$ & 0.465 & $\begin{array}{c}0.278^{* * *} \\
(5.455)\end{array}$ & $\begin{array}{c}0.499^{* * *} \\
(6.477)\end{array}$ & 0.647 & \\
\hline Long & AA & $\begin{array}{c}0.018 \\
(1.025)\end{array}$ & 0.030 & $\begin{array}{l}0.040^{*} \\
(1.852)\end{array}$ & 0.151 & $\begin{array}{c}0.017 \\
(1.302)\end{array}$ & $\begin{array}{l}0.042^{* *} \\
(2.274)\end{array}$ & 0.184 & 0.503 \\
\hline Long & $\mathrm{A}$ & $\begin{array}{c}0.022 \\
(1.118)\end{array}$ & 0.027 & $\begin{array}{c}0.065^{* *} \\
(2.194)\end{array}$ & 0.231 & $\begin{array}{l}0.034^{*} \\
(1.936)\end{array}$ & $\begin{array}{c}0.069^{* * *} \\
(2.909)\end{array}$ & 0.295 & \\
\hline Long & $\mathrm{BBB}$ & $\begin{array}{c}-0.074 \\
(-1.243)\end{array}$ & 0.031 & $\begin{array}{c}0.157^{* * *} \\
(5.208)\end{array}$ & 0.136 & $\begin{array}{l}-0.045 \\
(-0.896)\end{array}$ & $\begin{array}{c}0.153^{* * *} \\
(5.493)\end{array}$ & 0.149 & \\
\hline Long & $\mathrm{BB}$ & $\begin{array}{c}0.103 \\
(1.550)\end{array}$ & 0.066 & $\begin{array}{c}0.226^{* * *} \\
(4.321)\end{array}$ & 0.302 & $\begin{array}{c}0.124^{* * *} \\
(2.910)\end{array}$ & $\begin{array}{c}0.240^{* * * *} \\
(5.855)\end{array}$ & 0.394 & \\
\hline Long & $\mathrm{B}$ & $\begin{array}{l}0.211^{*} \\
(1.771)\end{array}$ & 0.046 & $\begin{array}{c}0.676^{* * *} \\
(2.819)\end{array}$ & 0.448 & $\begin{array}{c}0.362^{* * *} \\
(3.662)\end{array}$ & $\begin{array}{c}0.722^{* * *} \\
(4.303)\end{array}$ & 0.591 & \\
\hline Total & & & & & & & & & 0.482 \\
\hline
\end{tabular}

Notes: This table reports quarterly time series regressions of each of the 15 residuals of quarterly credit spread changes (in percentage), for cohorts based on time-to-maturity and credit rating, on $\Delta$ Inventory ${ }^{A}$ (in panel A), on $\Delta$ Distress (in panel B), and on both (in panel C). Robust t-statistics based on Newey and West (1987) standard errors using the optimal bandwidth choice in Andrews (1991) are reported in parentheses. Significance levels are represented by ${ }^{*} \mathrm{p}<0.1$, ${ }^{* *} \mathrm{p}<0.05$, and ${ }^{* * *} \mathrm{p}<0.01$ with $\mathrm{p}$ as the p-value. The last column reports the fraction of the total variation of residuals that is accounted for by $\Delta$ Inventory $^{A}$ and $\Delta$ Distress, denoted as FVE and computed as in (3) for short, medium, and long term bonds, as well as all bonds. The sample period is from 2005:Q1 through 2015:Q2. 
Table 5: Regressions of Credit Spread Changes Residuals on Liquidity Factor

\begin{tabular}{|c|c|c|c|c|c|c|c|c|c|}
\hline \multicolumn{2}{|c|}{ Groups } & \multicolumn{3}{|c|}{ A: $\Delta$ ILiq } & \multicolumn{5}{|c|}{ B: $\Delta$ Inventory ${ }^{A}+\Delta$ Distress $+\Delta \mathrm{ILiq}$} \\
\hline Maturity & Rating & $\Delta \mathrm{ILiq}$ & $\mathrm{R}_{a d j}^{2}$ & FVE & $\Delta$ Inventory $^{A}$ & $\Delta$ Distress & $\Delta \mathrm{ILiq}$ & $\mathrm{R}_{a d j}^{2}$ & FVE \\
\hline \multirow[t]{2}{*}{ Short } & AA & $0.053^{* * *}$ & 0.181 & 0.028 & 0.027 & $0.041^{* * *}$ & $0.041^{* * *}$ & 0.318 & 0.385 \\
\hline & & $(3.299)$ & & & $(1.466)$ & $(4.686)$ & $(3.872)$ & & \\
\hline \multirow[t]{2}{*}{ Short } & $\mathrm{A}$ & 0.032 & 0.044 & & $0.034^{* *}$ & $0.074^{* * *}$ & 0.011 & 0.302 & \\
\hline & & $(1.083)$ & & & $(1.993)$ & $(4.978)$ & $(1.037)$ & & \\
\hline \multirow[t]{2}{*}{ Short } & $\mathrm{BBB}$ & 0.042 & 0.039 & & $0.046^{* *}$ & $0.131^{* * *}$ & 0.005 & 0.411 & \\
\hline & & $(0.795)$ & & & $(2.231)$ & $(5.044)$ & $(0.394)$ & & \\
\hline \multirow[t]{2}{*}{ Short } & $\mathrm{BB}$ & 0.056 & 0.023 & & $0.095^{* *}$ & $0.203^{* * *}$ & 0.002 & 0.337 & \\
\hline & & $(0.960)$ & & & $(2.164)$ & $(5.021)$ & $(0.061)$ & & \\
\hline \multirow[t]{2}{*}{ Short } & B & 0.037 & 0.003 & & $0.292^{* * *}$ & $0.387^{* * *}$ & -0.060 & 0.390 & \\
\hline & & $(0.207)$ & & & $(3.801)$ & $(3.747)$ & $(-1.290)$ & & \\
\hline \multirow[t]{2}{*}{ Medium } & AA & $0.034^{* * *}$ & 0.070 & 0.023 & 0.011 & $0.042^{* * *}$ & 0.022 & 0.167 & 0.555 \\
\hline & & $(2.769)$ & & & $(0.626)$ & $(3.381)$ & (1.628) & & \\
\hline \multirow[t]{2}{*}{ Medium } & $\mathrm{A}$ & 0.048 & 0.080 & & $0.048^{* *}$ & $0.085^{* * *}$ & $0.025^{*}$ & 0.363 & \\
\hline & & $(1.346)$ & & & $(2.235)$ & $(4.157)$ & (1.850) & & \\
\hline \multirow[t]{2}{*}{ Medium } & $\mathrm{BBB}$ & 0.045 & 0.034 & & $0.075^{* *}$ & $0.144^{* * *}$ & 0.006 & 0.411 & \\
\hline & & $(0.705)$ & & & $(2.558)$ & $(4.320)$ & $(0.356)$ & & \\
\hline \multirow[t]{2}{*}{ Medium } & $\mathrm{BB}$ & 0.027 & 0.004 & & $0.128^{* * *}$ & $0.263^{* * *}$ & -0.043 & 0.424 & \\
\hline & & $(0.393)$ & & & $(3.000)$ & $(5.804)$ & $(-0.764)$ & & \\
\hline \multirow[t]{2}{*}{ Medium } & B & 0.101 & 0.022 & & $0.277 * * *$ & $0.509^{* * *}$ & -0.036 & 0.650 & \\
\hline & & $(0.475)$ & & & $(5.322)$ & $(6.817)$ & $(-0.854)$ & & \\
\hline \multirow[t]{2}{*}{ Long } & $\mathrm{AA}$ & $0.042^{* *}$ & 0.168 & 0.039 & 0.017 & $0.033^{* * *}$ & $0.033^{* * *}$ & 0.280 & 0.508 \\
\hline & & $(2.543)$ & & & (1.448) & $(2.784)$ & $(4.728)$ & & \\
\hline \multirow[t]{2}{*}{ Long } & $\mathrm{A}$ & $0.049 *$ & 0.130 & & $0.034^{* *}$ & $0.059 * * *$ & $0.033^{* *}$ & 0.349 & \\
\hline & & (1.827) & & & $(2.139)$ & $(3.366)$ & $(2.562)$ & & \\
\hline \multirow[t]{2}{*}{ Long } & $\mathrm{BBB}$ & 0.065 & 0.023 & & -0.044 & $0.147^{* * *}$ & 0.018 & 0.151 & \\
\hline & & (1.478) & & & $(-0.896)$ & $(5.882)$ & $(0.670)$ & & \\
\hline \multirow[t]{2}{*}{ Long } & $\mathrm{BB}$ & -0.002 & 0.000 & & $0.122^{* * *}$ & $0.261^{* * *}$ & $-0.072 *$ & 0.422 & \\
\hline & & $(-0.032)$ & & & $(2.829)$ & $(6.263)$ & $(-1.775)$ & & \\
\hline \multirow[t]{2}{*}{ Long } & B & 0.214 & 0.045 & & $0.362^{* * *}$ & $0.716^{* * *}$ & 0.020 & 0.592 & \\
\hline & & $(0.687)$ & & & $(3.671)$ & $(4.461)$ & $(0.236)$ & & \\
\hline Total & & & & 0.032 & & & & & 0.488 \\
\hline
\end{tabular}

Notes: This table reports quarterly time series regressions of each of the 15 residuals of quarterly credit spread changes (in percentage), for cohorts based on time-to-maturity and credit rating, on $\Delta$ ILiq in univariate regressions (in panel A) and in multivariate regressions along with $\Delta$ Inventory $^{A}$ and $\Delta$ Distress (in panel B), respectively. Robust t-statistics based on Newey and West (1987) standard errors using the optimal bandwidth choice in Andrews (1991) are reported in parentheses. Significance levels are represented by * $\mathrm{p}<0.1,{ }^{* *} \mathrm{p}<0.05$, and ${ }^{* * *} \mathrm{p}<0.01$ with $\mathrm{p}$ as the $\mathrm{p}$-value. The last column in each panel reports the fraction of the total variation of residuals that is accounted for, denoted as FVE and computed as in (3) for short, medium, and long term bonds, as well as all bonds. The sample period is from 2005:Q1 through 2015:Q2. 


\section{Table 6: Quarterly Series by Leverage Cohort}

\begin{tabular}{|c|c|c|c|c|c|c|c|c|c|}
\hline \multicolumn{2}{|c|}{ Groups } & \multicolumn{2}{|c|}{ A: Sample } & \multicolumn{2}{|c|}{ B: PC } & \multicolumn{4}{|c|}{ C: Regressions of Residuals } \\
\hline Maturity & Leverage & Bond \# & Obs & First & Second & $\Delta$ Inventory $^{A}$ & $\Delta$ Distress & $\mathrm{R}_{a d j}^{2}$ & FVE \\
\hline Short & $<15 \%$ & 295 & 3434 & 0.095 & -0.004 & $\begin{array}{c}0.048^{* * *} \\
(2.773)\end{array}$ & $\begin{array}{c}0.087^{* * *} \\
(3.579)\end{array}$ & 0.322 & 0.324 \\
\hline Short & $15-25 \%$ & 476 & 5714 & 0.137 & -0.0002 & $\begin{array}{c}0.064^{* *} \\
(2.271)\end{array}$ & $\begin{array}{c}0.119^{* * *} \\
(4.030)\end{array}$ & 0.259 & \\
\hline Short & $25-35 \%$ & 414 & 4691 & 0.177 & -0.035 & $\begin{array}{c}0.109^{* * *} \\
(3.322)\end{array}$ & $\begin{array}{c}0.151^{* * *} \\
(3.870)\end{array}$ & 0.323 & \\
\hline Short & $35-45 \%$ & 212 & 2112 & 0.271 & -0.025 & $\begin{array}{c}0.144^{* *} \\
(2.515)\end{array}$ & $\begin{array}{c}0.238^{* * *} \\
(3.962)\end{array}$ & 0.293 & \\
\hline Short & $>45 \%$ & 249 & 2350 & 0.442 & -0.163 & $\begin{array}{c}0.273^{* * *} \\
(3.296)\end{array}$ & $\begin{array}{c}0.393^{* * *} \\
(3.446)\end{array}$ & 0.342 & \\
\hline Medium & $<15 \%$ & 276 & 2687 & 0.105 & -0.018 & $\begin{array}{l}0.056^{* *} \\
(2.575)\end{array}$ & $\begin{array}{c}0.099^{* * *} \\
(3.091)\end{array}$ & 0.336 & 0.547 \\
\hline Medium & $15-25 \%$ & 453 & 4055 & 0.188 & 0.029 & $\begin{array}{c}0.103^{* * *} \\
(3.108)\end{array}$ & $\begin{array}{c}0.237^{* * *} \\
(6.545)\end{array}$ & 0.563 & \\
\hline Medium & $25-35 \%$ & 436 & 3919 & 0.217 & 0.001 & $\begin{array}{c}0.127^{* * *} \\
(3.436)\end{array}$ & $\begin{array}{c}0.252^{* * *} \\
(5.382)\end{array}$ & 0.526 & \\
\hline Medium & $35-45 \%$ & 255 & 2331 & 0.269 & 0.062 & $\begin{array}{c}0.150^{* * *} \\
(2.881)\end{array}$ & $\begin{array}{c}0.279^{* * *} \\
(5.288)\end{array}$ & 0.385 & \\
\hline Medium & $>45 \%$ & 263 & 2269 & 0.441 & -0.028 & $\begin{array}{c}0.356^{* * *} \\
(4.793)\end{array}$ & $\begin{array}{c}0.544^{* * *} \\
(4.474)\end{array}$ & 0.623 & \\
\hline Long & $<15 \%$ & 361 & 5059 & 0.079 & 0.004 & $\begin{array}{c}0.036^{* *} \\
(2.008)\end{array}$ & $\begin{array}{c}0.078^{* *} \\
(2.360)\end{array}$ & 0.322 & 0.392 \\
\hline Long & $15-25 \%$ & 506 & 7063 & 0.102 & 0.978 & $\begin{array}{c}-0.047 \\
(-0.739)\end{array}$ & $\begin{array}{c}0.191^{* * *} \\
(4.370)\end{array}$ & 0.112 & \\
\hline Long & $25-35 \%$ & 418 & 6038 & 0.129 & 0.029 & $\begin{array}{c}0.074^{* *} \\
(2.459)\end{array}$ & $\begin{array}{c}0.136^{* * *} \\
(3.485)\end{array}$ & 0.405 & \\
\hline Long & $35-45 \%$ & 174 & 1885 & 0.190 & 0.051 & $\begin{array}{c}0.139^{* * *} \\
(4.231)\end{array}$ & $\begin{array}{c}0.227^{* * *} \\
(6.776)\end{array}$ & 0.523 & \\
\hline Long & $>45 \%$ & 166 & 1791 & 0.493 & -0.075 & $\begin{array}{c}0.322^{* * *} \\
(3.169)\end{array}$ & $\begin{array}{c}0.554^{* * *} \\
(3.220)\end{array}$ & 0.513 & \\
\hline Pct Expla & & & & 0.781 & 0.102 & & & & 0.422 \\
\hline
\end{tabular}

Notes: This table reports results using 15 cohorts based on time-to-maturity and firm leverage. Panel A reports the number of bonds and observations for each cohort. Panel B reports the loadings of the first two PCs on the 15 regression residuals and the fraction of total variation these two PCs account for. Panel C reports quarterly time series regressions of each of the 15 residuals of quarterly credit spread changes (in percentage) on $\Delta$ Inventory $^{A}$ (in panel $\mathrm{A}$ ) and $\Delta$ Distress, with robust t-statistics based on Newey and West (1987) standard errors using the optimal bandwidth choice in Andrews (1991) reported in parentheses. Significance levels are represented by ${ }^{*} \mathrm{p}<0.1,{ }^{* *} \mathrm{p}<0.05$, and ${ }^{* * *} \mathrm{p}<0.01$ with $\mathrm{p}$ as the $\mathrm{p}$-value. The last column of Panel $\mathrm{C}$ reports the fraction of the total variation of residuals that is accounted for by the two intermediary factors, denoted as FVE and computed as in (3) for short, medium, and long term bonds, as well as all bonds. The sample period is from 2005:Q1 through 2015:Q2. 


\section{Table 7: Monthly Series by Rating Group}

\begin{tabular}{|c|c|c|c|c|c|c|c|c|c|c|}
\hline \multicolumn{2}{|c|}{ Groups } & \multicolumn{3}{|c|}{ A: Individual Bond Regressions } & \multicolumn{2}{|c|}{ B: PC } & \multicolumn{4}{|c|}{ C: Regression of Residuals } \\
\hline Maturity & Rating & Bond \# & Obs & $\mathrm{R}_{a d j}^{2}$ & First & Second & $\Delta$ Inventory ${ }^{A}$ & $\Delta$ Distress & $\mathrm{R}_{a d j}^{2}$ & FVE \\
\hline Short & $\mathrm{AA}$ & 87 & 2611 & 0.133 & 0.065 & 0.124 & $\begin{array}{l}0.016^{*} \\
(1.801)\end{array}$ & $\begin{array}{c}0.014 \\
(1.285)\end{array}$ & 0.045 & 0.192 \\
\hline Short & A & 525 & 15871 & 0.164 & 0.093 & 0.142 & $\begin{array}{c}0.012 \\
(1.494)\end{array}$ & $\begin{array}{c}0.037^{* * *} \\
(3.131)\end{array}$ & 0.129 & \\
\hline Short & BBB & 881 & 25114 & 0.187 & 0.15 & 0.16 & $\begin{array}{c}0.009 \\
(0.797)\end{array}$ & $\begin{array}{c}0.053^{* *} \\
(2.206)\end{array}$ & 0.131 & \\
\hline Short & $\mathrm{BB}$ & 401 & 7835 & 0.324 & 0.291 & 0.251 & $\begin{array}{l}0.042^{* *} \\
(2.095)\end{array}$ & $\begin{array}{c}0.127^{* * *} \\
(3.818)\end{array}$ & 0.195 & \\
\hline Short & B & 485 & 10061 & 0.347 & 0.48 & 0.065 & $\begin{array}{l}0.077^{* *} \\
(2.097)\end{array}$ & $\begin{array}{c}0.172^{* * *} \\
(4.422)\end{array}$ & 0.191 & \\
\hline Medium & $\mathrm{AA}$ & 73 & 1680 & 0.171 & 0.061 & 0.106 & $\begin{array}{c}0.013 \\
(1.530)\end{array}$ & $\begin{array}{c}0.022^{* *} \\
(2.338)\end{array}$ & 0.077 & 0.138 \\
\hline Medium & A & 448 & 9885 & 0.166 & 0.087 & 0.153 & $\begin{array}{l}0.016^{* *} \\
(2.022)\end{array}$ & $\begin{array}{c}0.016 \\
(1.029)\end{array}$ & 0.043 & \\
\hline Medium & BBB & 880 & 18088 & 0.218 & 0.146 & 0.21 & $\begin{array}{c}0.023^{*} \\
(1.893)\end{array}$ & $\begin{array}{c}0.046^{* *} \\
(2.038)\end{array}$ & 0.104 & \\
\hline Medium & $\mathrm{BB}$ & 491 & 8989 & 0.399 & 0.274 & 0.218 & $\begin{array}{c}0.061^{* * *} \\
(3.482)\end{array}$ & $\begin{array}{c}0.122^{* * *} \\
(2.875)\end{array}$ & 0.246 & \\
\hline Medium & B & 593 & 13111 & 0.392 & 0.402 & 0.163 & $\begin{array}{c}0.047 \\
(1.353) \\
\end{array}$ & $\begin{array}{l}0.106^{*} \\
(1.828)\end{array}$ & 0.091 & \\
\hline Long & $\mathrm{AA}$ & 119 & 4495 & 0.197 & 0.058 & 0.117 & $\begin{array}{c}0.014^{* *} \\
(1.966)\end{array}$ & $\begin{array}{c}0.011 \\
(0.965)\end{array}$ & 0.046 & 0.235 \\
\hline Long & A & 638 & 24132 & 0.216 & 0.082 & 0.131 & $\begin{array}{c}0.015^{* *} \\
(2.082)\end{array}$ & $\begin{array}{l}0.024^{*} \\
(1.731)\end{array}$ & 0.092 & \\
\hline Long & BBB & 1049 & 33504 & 0.260 & 0.114 & 0.262 & $\begin{array}{c}0.015 \\
(1.422)\end{array}$ & $\begin{array}{l}0.033^{*} \\
(1.946)\end{array}$ & 0.032 & \\
\hline Long & $\mathrm{BB}$ & 352 & 6768 & 0.352 & 0.232 & 0.204 & $\begin{array}{c}0.029 \\
(1.450)\end{array}$ & $\begin{array}{c}0.070^{* * *} \\
(2.935)\end{array}$ & 0.101 & \\
\hline Long & B & 277 & 5715 & 0.361 & 0.551 & -0.761 & $\begin{array}{r}0.098^{* *} \\
(1.986)\end{array}$ & $\begin{array}{c}0.261^{* * *} \\
(3.070)\end{array}$ & 0.264 & \\
\hline Pct Expla & hed & & & & 0.757 & 0.092 & & & & 0.196 \\
\hline
\end{tabular}

Notes: This table reports results at the monthly frequency using 15 cohorts based on time-to-maturity and credit rating. Panel A reports the number of bonds, number of observations, and mean adjusted $R^{2} \mathrm{~s}$ for each cohort. Panel B reports the loadings of the first two PCs on the 15 regression residuals and the fraction of total variation these two PCs account for. Panel C reports monthly time series regressions of each of the 15 residuals of monthly credit spread changes (in percentage) on $\Delta$ Inventory $^{A}$ and $\Delta$ Distress, with robust t-statistics based on Newey and West (1987) standard errors using the optimal bandwidth choice in Andrews (1991) reported in parentheses. Significance levels are represented by ${ }^{*} \mathrm{p}<0.1,{ }^{* *} \mathrm{p}<0.05$, and *** $\mathrm{p}<0.01$ with $\mathrm{p}$ as the $\mathrm{p}$-value. The last column of Panel $\mathrm{C}$ reports the fraction of the total variation of residuals that is accounted for by the two intermediary factors, denoted as FVE and computed as in (3) for short, medium, and long term bonds, as well as all bonds. The sample period is from 2005:Q1 through 2015:Q2. 
Table 8: Measures of Intermediary Distress

\begin{tabular}{|c|c|c|c|c|c|c|c|c|}
\hline \multicolumn{2}{|c|}{ Groups } & \multicolumn{2}{|c|}{$\mathrm{A}: \Delta N o i s e$} & \multicolumn{2}{|c|}{ B: $\Delta \mathrm{NLev}^{\mathrm{HKM}}$} & \multicolumn{3}{|c|}{ C: $\Delta$ Noise $+\Delta$ NLev $^{\text {HKM }}$} \\
\hline Maturity & Rating & $\Delta$ Noise & $\mathrm{R}_{a d j}^{2}$ & $\Delta \mathrm{NLev}^{\mathrm{HKM}}$ & $\mathrm{R}_{a d j}^{2}$ & $\Delta$ Noise & $\Delta \mathrm{NLev}^{\mathrm{HKM}}$ & $\mathrm{R}_{a d j}^{2}$ \\
\hline \multirow[t]{2}{*}{ Short } & $\mathrm{AA}$ & $0.043^{*}$ & 0.113 & 0.021 & 0.026 & $0.042^{*}$ & 0.005 & 0.114 \\
\hline & & $(1.683)$ & & $(0.845)$ & & $(1.883)$ & $(0.199)$ & \\
\hline \multirow[t]{2}{*}{ Short } & $\mathrm{A}$ & $0.082^{* *}$ & 0.225 & 0.029 & 0.028 & $0.083^{* *}$ & -0.004 & 0.226 \\
\hline & & $(2.429)$ & & $(1.026)$ & & $(2.494)$ & $(-0.141)$ & \\
\hline \multirow[t]{2}{*}{ Short } & $\mathrm{BBB}$ & $0.132^{* * *}$ & 0.306 & $0.069^{*}$ & 0.083 & $0.124^{* * *}$ & 0.021 & 0.312 \\
\hline & & $(3.368)$ & & $(1.682)$ & & $(2.973)$ & $(0.619)$ & \\
\hline \multirow[t]{2}{*}{ Short } & $\mathrm{BB}$ & $0.320 * * *$ & 0.399 & 0.010 & 0.000 & $0.373^{* * *}$ & $-0.135^{*}$ & 0.459 \\
\hline & & $(2.804)$ & & $(0.097)$ & & $(3.610)$ & $(-1.958)$ & \\
\hline \multirow[t]{2}{*}{ Short } & $\mathrm{B}$ & $0.389^{* * *}$ & 0.221 & 0.206 & 0.062 & $0.363^{* * *}$ & 0.065 & 0.226 \\
\hline & & $(2.762)$ & & (1.115) & & $(2.668)$ & $(0.399)$ & \\
\hline \multirow[t]{2}{*}{ Medium } & $\mathrm{AA}$ & $0.058^{* * *}$ & 0.188 & 0.023 & 0.029 & $0.058^{* *}$ & 0.001 & 0.188 \\
\hline & & $(2.614)$ & & (1.363) & & $(2.358)$ & $(0.030)$ & \\
\hline \multirow[t]{2}{*}{ Medium } & $\mathrm{A}$ & $0.077^{* *}$ & 0.182 & $0.070^{* *}$ & 0.152 & 0.058 & 0.047 & 0.241 \\
\hline & & $(1.980)$ & & $(2.180)$ & & $(1.431)$ & (1.601) & \\
\hline \multirow[t]{2}{*}{ Medium } & $\mathrm{BBB}$ & $0.127 * *$ & 0.224 & $0.115^{* *}$ & 0.184 & $0.097^{*}$ & $0.077^{*}$ & 0.295 \\
\hline & & $(2.376)$ & & $(2.284)$ & & $(1.868)$ & $(1.771)$ & \\
\hline \multirow[t]{2}{*}{ Medium } & $\mathrm{BB}$ & $0.310^{* * *}$ & 0.448 & 0.107 & 0.053 & $0.316^{* * *}$ & -0.016 & 0.449 \\
\hline & & $(3.550)$ & & $(1.224)$ & & $(2.951)$ & $(-0.286)$ & \\
\hline \multirow[t]{2}{*}{ Medium } & B & $0.432^{* * *}$ & 0.304 & $0.422^{* * *}$ & 0.290 & $0.316^{* *}$ & $0.300 * * *$ & 0.429 \\
\hline & & $(2.669)$ & & $(3.070)$ & & $(2.352)$ & $(2.700)$ & \\
\hline \multirow[t]{2}{*}{ Long } & $\mathrm{AA}$ & 0.034 & 0.076 & 0.021 & 0.030 & 0.030 & 0.010 & 0.081 \\
\hline & & (1.102) & & $(0.734)$ & & $(1.425)$ & $(0.367)$ & \\
\hline \multirow[t]{2}{*}{ Long } & $\mathrm{A}$ & $0.066^{*}$ & 0.186 & 0.043 & 0.079 & $0.058^{*}$ & 0.021 & 0.202 \\
\hline & & $(1.815)$ & & (1.354) & & $(1.715)$ & $(0.711)$ & \\
\hline \multirow[t]{2}{*}{ Long } & $\mathrm{BBB}$ & $0.177^{* * *}$ & 0.155 & $0.114^{* * *}$ & 0.064 & $0.156^{* * *}$ & $0.054^{*}$ & 0.167 \\
\hline & & $(3.037)$ & & $(4.169)$ & & $(2.762)$ & (1.806) & \\
\hline \multirow[t]{2}{*}{ Long } & $\mathrm{BB}$ & $0.291^{* * *}$ & 0.457 & 0.114 & 0.071 & $0.290 * * *$ & 0.002 & 0.457 \\
\hline & & $(4.632)$ & & $(1.423)$ & & $(3.664)$ & $(0.047)$ & \\
\hline \multirow[t]{2}{*}{ Long } & B & $0.672^{* * *}$ & 0.374 & $0.566^{* *}$ & 0.265 & $0.533^{* *}$ & $0.359^{*}$ & 0.465 \\
\hline & & $(2.851)$ & & $(2.412)$ & & $(2.282)$ & $(1.769)$ & \\
\hline FVE & & & 0.321 & & 0.168 & & & 0.380 \\
\hline
\end{tabular}

Notes: This table reports quarterly time series regressions of each of the 15 residuals of quarterly credit spread changes (in percentage), for cohorts based on time-to-maturity and credit rating, on $\Delta$ Noise (in panel A), on $\Delta \mathrm{NLev}^{\mathrm{HKM}}$ (in panel B), and on both (in panel C). Robust t-statistics based on Newey and West (1987) standard errors using the optimal bandwidth choice in Andrews (1991) are reported in parentheses. Significance levels are represented by ${ }^{*} \mathrm{p}<0.1,{ }^{* *} \mathrm{p}<0.05$, and ${ }^{* * *} \mathrm{p}<0.01$ with $\mathrm{p}$ as the $\mathrm{p}$-value. The last row reports the fraction of the total variation of residuals that is accounted for by $\Delta$ Noise, $\Delta \mathrm{NLev}^{\mathrm{HKM}}$ and both, respectively, denoted as FVE and computed as in (3) for all cohorts. The sample period is from 2005:Q1 through 2015:Q2. 


\section{Table 9: Groups by Trading Volume}

\begin{tabular}{|c|c|c|c|c|c|c|c|}
\hline \multicolumn{2}{|c|}{ Groups } & \multicolumn{3}{|c|}{ A: Sample Summary } & \multicolumn{3}{|c|}{ B: Regressions of Residuals } \\
\hline Leverage & Trd Volume & TrdVolume (\$ million) & Bond\# & Obs & $\Delta$ Distress & $\Delta$ Inventory $^{A}$ & $\mathrm{R}_{a d j}^{2}$ \\
\hline AA & Low & 2.462 & 92 & 530 & $\begin{array}{c}0.036 \\
(1.517)\end{array}$ & $\begin{array}{c}0.018 \\
(0.934)\end{array}$ & 0.067 \\
\hline $\mathrm{AA}$ & Medium & 17.779 & 113 & 796 & $\begin{array}{c}0.051^{* * *} \\
(3.100)\end{array}$ & $\begin{array}{c}0.017 \\
(1.040)\end{array}$ & 0.177 \\
\hline AA & High & 136.25 & 129 & 1084 & $\begin{array}{c}0.038 \\
(1.161)\end{array}$ & $\begin{array}{c}0.016 \\
(0.938)\end{array}$ & 0.098 \\
\hline A & Low & 1.995 & 684 & 6201 & $\begin{array}{c}0.072^{* * *} \\
(2.892)\end{array}$ & $\begin{array}{l}0.039^{*} \\
(1.803)\end{array}$ & 0.260 \\
\hline A & Medium & 16.882 & 741 & 4700 & $\begin{array}{c}0.086^{* * *} \\
(3.300)\end{array}$ & $\begin{array}{l}0.045^{*} \\
(1.907)\end{array}$ & 0.302 \\
\hline A & High & 110.411 & 699 & 4246 & $\begin{array}{l}0.073^{*} \\
(1.759)\end{array}$ & $\begin{array}{c}0.035 \\
(1.509)\end{array}$ & 0.207 \\
\hline BBB & Low & 2.011 & 1199 & 9465 & $\begin{array}{c}0.146^{* * *} \\
(3.556)\end{array}$ & $\begin{array}{c}0.023 \\
(0.564)\end{array}$ & 0.085 \\
\hline BBB & Medium & 17.056 & 1209 & 7401 & $\begin{array}{c}0.174^{* * *} \\
(5.770)\end{array}$ & $\begin{array}{l}0.057^{*} \\
(1.848)\end{array}$ & 0.426 \\
\hline BBB & High & 106.026 & 1137 & 6405 & $\begin{array}{c}0.150^{* * * *} \\
(2.698)\end{array}$ & $\begin{array}{l}0.070^{* *} \\
(2.329)\end{array}$ & 0.355 \\
\hline BB & Low & 2.584 & 431 & 1973 & $\begin{array}{c}0.251^{* * *} \\
(4.007)\end{array}$ & $\begin{array}{c}0.160^{* * *} \\
(2.688)\end{array}$ & 0.369 \\
\hline BB & Medium & 17.777 & 471 & 2435 & $\begin{array}{c}0.262^{* * *} \\
(4.540)\end{array}$ & $\begin{array}{c}0.155^{* *} \\
(2.118)\end{array}$ & 0.358 \\
\hline BB & High & 100.298 & 451 & 2303 & $\begin{array}{c}0.227^{* * *} \\
(4.774)\end{array}$ & $\begin{array}{l}0.123^{*} \\
(1.732)\end{array}$ & 0.279 \\
\hline B & Low & 2.36 & 412 & 2282 & $\begin{array}{c}0.450^{* * *} \\
(4.502)\end{array}$ & $\begin{array}{c}0.317^{* * *} \\
(3.473)\end{array}$ & 0.411 \\
\hline B & Medium & 17.342 & 468 & 2973 & $\begin{array}{c}0.526^{* * *} \\
(3.459)\end{array}$ & $\begin{array}{c}0.270^{* * *} \\
(2.996)\end{array}$ & 0.461 \\
\hline B & High & 89.654 & 437 & 2604 & $\begin{array}{c}0.586^{* * *} \\
(3.283)\end{array}$ & $\begin{array}{c}0.304^{* * *} \\
(3.155)\end{array}$ & 0.481 \\
\hline
\end{tabular}

Note: This table reports results using 15 cohorts based on credit rating and trading volume (dollar value of the total trading volume in the last month of a quarter). Panel A reports the total dollar trading volume in \$millions, number of bonds, and number of observations for each cohort. Panel B reports quarterly time series regressions of each of the 15 residuals of quarterly credit spread changes (in percentage) on $\Delta$ Inventory ${ }^{A}$ and $\Delta$ Distress, with robust t-statistics based on Newey and West (1987) standard errors using the optimal bandwidth choice in Andrews (1991) reported in parentheses. Significance levels are represented by $* \mathrm{p}<0.1$, ${ }^{* *} \mathrm{p}<0.05$, and ${ }^{* * *} \mathrm{p}<0.01$ with p as the p-value. The sample period is from 2005:Q1 through 2015:Q2. 
Table 10: Inventories of HY vs IG Bonds

\begin{tabular}{|c|c|c|c|c|c|c|c|}
\hline \multicolumn{2}{|c|}{ Groups } & \multicolumn{3}{|c|}{ A: $\Delta$ Inventory ${ }^{H Y}$} & \multicolumn{3}{|c|}{ B: $\Delta$ Inventory $^{I G}$} \\
\hline Maturity & Rating & $\Delta$ Distress & $\Delta$ Inventory $^{H Y}$ & $\mathrm{R}_{a d j}^{2}$ & $\Delta$ Distress & $\Delta$ Inventory $^{I G}$ & $\mathrm{R}_{a d j}^{2}$ \\
\hline \multirow[t]{2}{*}{ Short } & AA & 0.041 & 0.016 & 0.107 & 0.042 & 0.024 & 0.126 \\
\hline & & $(1.472)$ & $(0.894)$ & & $(1.627)$ & $(1.340)$ & \\
\hline \multirow[t]{2}{*}{ Short } & $\mathrm{A}$ & $0.071^{* *}$ & $0.042^{*}$ & 0.218 & $0.070^{* *}$ & 0.021 & 0.174 \\
\hline & & $(2.492)$ & $(1.748)$ & & $(2.439)$ & $(1.073)$ & \\
\hline \multirow[t]{2}{*}{ Short } & $\mathrm{BBB}$ & $0.127^{* * *}$ & $0.062^{* *}$ & 0.327 & $0.126^{* * *}$ & 0.033 & 0.282 \\
\hline & & $(3.294)$ & $(1.979)$ & & $(3.327)$ & $(1.160)$ & \\
\hline \multirow[t]{2}{*}{ Short } & $\mathrm{BB}$ & $0.210^{* * *}$ & 0.119 & 0.207 & $0.215^{* * *}$ & $0.121^{* *}$ & 0.210 \\
\hline & & $(2.994)$ & $(1.278)$ & & $(3.876)$ & $(2.137)$ & \\
\hline \multirow[t]{2}{*}{ Short } & B & $0.376^{* *}$ & 0.191 & 0.238 & $0.391^{* * *}$ & $0.251^{* *}$ & 0.276 \\
\hline & & $(2.097)$ & $(1.373)$ & & $(2.735)$ & $(2.231)$ & \\
\hline \multirow[t]{2}{*}{ Medium } & AA & $0.052^{* * *}$ & 0.030 & 0.184 & $0.049^{* * *}$ & 0.002 & 0.136 \\
\hline & & $(3.561)$ & $(1.583)$ & & $(3.034)$ & $(0.132)$ & \\
\hline \multirow[t]{2}{*}{ Medium } & $\mathrm{A}$ & $0.093^{* *}$ & $0.053^{* *}$ & 0.325 & $0.090^{* *}$ & 0.016 & 0.252 \\
\hline & & $(2.319)$ & $(2.412)$ & & $(2.082)$ & $(0.697)$ & \\
\hline \multirow[t]{2}{*}{ Medium } & $\mathrm{BBB}$ & $0.154^{* * *}$ & $0.083^{* *}$ & 0.389 & $0.149^{* *}$ & 0.025 & 0.307 \\
\hline & & $(2.661)$ & $(2.522)$ & & $(2.443)$ & $(0.765)$ & \\
\hline \multirow[t]{2}{*}{ Medium } & $\mathrm{BB}$ & $0.264^{* * *}$ & $0.142^{* *}$ & 0.381 & $0.261^{* * *}$ & 0.076 & 0.319 \\
\hline & & $(4.082)$ & (1.971) & & $(4.628)$ & (1.569) & \\
\hline \multirow[t]{2}{*}{ Medium } & B & $0.538^{* * *}$ & $0.237^{* *}$ & 0.529 & $0.541^{* * *}$ & $0.197 * * *$ & 0.504 \\
\hline & & $(3.609)$ & $(2.368)$ & & $(4.190)$ & $(2.912)$ & \\
\hline \multirow[t]{2}{*}{ Long } & AA & 0.036 & $0.029^{* *}$ & 0.129 & 0.034 & 0.001 & 0.077 \\
\hline & & (1.137) & $(2.063)$ & & $(0.960)$ & $(0.051)$ & \\
\hline \multirow[t]{2}{*}{ Long } & $\mathrm{A}$ & $0.069^{*}$ & $0.033^{*}$ & 0.232 & $0.070^{*}$ & 0.025 & 0.215 \\
\hline & & $(1.816)$ & $(1.703)$ & & $(1.829)$ & $(1.335)$ & \\
\hline \multirow[t]{2}{*}{ Long } & BBB & $0.177^{* * *}$ & 0.012 & 0.156 & $0.175^{* * *}$ & -0.009 & 0.155 \\
\hline & & $(5.107)$ & $(0.280)$ & & $(4.966)$ & $(-0.348)$ & \\
\hline \multirow[t]{2}{*}{ Long } & $\mathrm{BB}$ & $0.256^{* * *}$ & $0.129^{*}$ & 0.412 & $0.255^{* * *}$ & $0.089^{*}$ & 0.369 \\
\hline & & $(4.416)$ & (1.888) & & $(5.734)$ & $(1.770)$ & \\
\hline \multirow[t]{2}{*}{ Long } & B & $0.772^{* * *}$ & $0.244^{*}$ & 0.526 & $0.788^{* * *}$ & $0.306^{* * *}$ & 0.554 \\
\hline & & $(2.991)$ & $(1.748)$ & & $(3.446)$ & $(2.693)$ & \\
\hline FVE & & & & 0.397 & & & 0.322 \\
\hline
\end{tabular}

Note: This table reports quarterly time series regressions of each of the 15 residuals of quarterly credit spread changes (in percentage), for cohorts based on time-to-maturity and credit rating, on $\Delta$ Inventory ${ }^{H Y}$ (in panel A), on $\Delta$ Inventory $^{I G}$ (in panel B). Robust t-statistics based on Newey and West (1987) standard errors using the optimal bandwidth choice in Andrews (1991) are reported in parentheses. Significance levels are represented by ${ }^{*} \mathrm{p}<0.1,{ }^{* *} \mathrm{p}<0.05$, and ${ }^{* * *} \mathrm{p}<0.01$ with $\mathrm{p}$ as the $\mathrm{p}$-value. The last row reports the fraction of the total variation of residuals that is accounted for, denoted as FVE and computed as in (3), for all cohorts. The sample period is from 2005:Q1 through 2015:Q2. 


\section{Table 11: Credit Default Swaps}

\begin{tabular}{|c|c|c|c|c|c|c|c|c|c|}
\hline \multicolumn{2}{|c|}{ Groups } & \multicolumn{2}{|c|}{ A: Sample } & \multicolumn{2}{|c|}{ B: PC } & \multicolumn{4}{|c|}{ C: Regression of Residuals } \\
\hline Maturity & Rating & Firm \# & Obs & First & Second & $\Delta$ Inventory $^{A}$ & $\Delta$ Distress & $\mathrm{R}_{a d j}^{2}$ & FVE \\
\hline $1 \mathrm{y}$ & AA & 20 & 939 & 0.039 & -0.029 & $\begin{array}{c}0.007 \\
(0.542)\end{array}$ & $\begin{array}{c}0.030^{* *} \\
(2.012)\end{array}$ & 0.121 & 0.375 \\
\hline $1 \mathrm{y}$ & A & 111 & 5742 & 0.041 & 0.042 & $\begin{array}{c}0.032^{* * *} \\
(4.218)\end{array}$ & $\begin{array}{c}0.040^{* * *} \\
(6.210)\end{array}$ & 0.463 & \\
\hline $1 \mathrm{y}$ & $\mathrm{BBB}$ & 200 & 7942 & 0.067 & 0.062 & $\begin{array}{c}0.048^{* * *} \\
(3.882)\end{array}$ & $\begin{array}{c}0.057^{* * *} \\
(5.025)\end{array}$ & 0.414 & \\
\hline $1 \mathrm{y}$ & $\mathrm{BB}$ & 128 & 2309 & 0.149 & 0.151 & $\begin{array}{c}0.099^{* * *} \\
(3.615)\end{array}$ & $\begin{array}{c}0.141^{* * *} \\
(7.691)\end{array}$ & 0.367 & \\
\hline $1 \mathrm{y}$ & B & 64 & 1377 & 0.651 & 0.686 & $\begin{array}{c}0.305^{* * *} \\
(2.721)\end{array}$ & $\begin{array}{c}0.492^{* *} \\
(2.521)\end{array}$ & 0.377 & \\
\hline $5 y$ & AA & 21 & 1140 & 0.031 & -0.010 & $\begin{array}{l}0.013^{*} \\
(1.725)\end{array}$ & $\begin{array}{c}0.026^{* * *} \\
(2.914)\end{array}$ & 0.185 & 0.354 \\
\hline $5 y$ & A & 112 & 5688 & 0.043 & 0.035 & $\begin{array}{c}0.030^{* * *} \\
(2.950)\end{array}$ & $\begin{array}{c}0.042^{* * *} \\
(7.351)\end{array}$ & 0.356 & \\
\hline $5 y$ & BBB & 208 & 7995 & 0.067 & 0.003 & $\begin{array}{c}0.046^{* * *} \\
(2.752)\end{array}$ & $\begin{array}{c}0.067^{* * *} \\
(4.479)\end{array}$ & 0.379 & \\
\hline $5 y$ & $\mathrm{BB}$ & 132 & 2377 & 0.127 & 0.072 & $\begin{array}{c}0.007 \\
(0.176)\end{array}$ & $\begin{array}{c}0.112^{* * *} \\
(4.294)\end{array}$ & 0.165 & \\
\hline $5 y$ & B & 71 & 1601 & 0.583 & -0.643 & $\begin{array}{c}0.287^{* * *} \\
(2.666)\end{array}$ & $\begin{array}{c}0.440^{* * *} \\
(2.912)\end{array}$ & 0.376 & \\
\hline $10 y$ & AA & 20 & 1117 & 0.023 & -0.018 & $\begin{array}{c}0.011 \\
(1.375)\end{array}$ & $\begin{array}{c}0.012 \\
(1.440)\end{array}$ & 0.063 & 0.395 \\
\hline $10 y$ & A & 111 & 5611 & 0.036 & 0.035 & $\begin{array}{c}0.025^{* *} \\
(2.228)\end{array}$ & $\begin{array}{c}0.039^{* * *} \\
(6.795)\end{array}$ & 0.314 & \\
\hline $10 y$ & BBB & 198 & 8071 & 0.055 & -0.001 & $\begin{array}{c}0.036^{* *} \\
(2.263)\end{array}$ & $\begin{array}{c}0.058^{* * *} \\
(5.811)\end{array}$ & 0.350 & \\
\hline $10 y$ & $\mathrm{BB}$ & 127 & 2426 & 0.094 & 0.039 & $\begin{array}{c}0.026 \\
(0.792)\end{array}$ & $\begin{array}{c}0.074^{* * *} \\
(4.387)\end{array}$ & 0.122 & \\
\hline $10 y$ & B & 65 & 1409 & 0.413 & -0.277 & $\begin{array}{c}0.206^{* *} \\
(2.515)\end{array}$ & $\begin{array}{c}0.354^{* * *} \\
(2.667)\end{array}$ & 0.438 & \\
\hline
\end{tabular}

Pct Explained

$\begin{array}{ll}0.830 & 0.070\end{array}$

Note: This table reports results using 15 cohorts of CDS based on the CDS maturity and credit rating of the underlying entity. Panel A reports the number of firms and observations for each cohort. Panel B reports the loadings of the first two PCs on the 15 regression residuals (computed from time series regressions of quarterly CDS spread changes in percentage similar to (1)) and the fraction of total variation these two PCs account for. Panel C reports quarterly time series regressions of each of the 15 residuals of quarterly CDS spread changes (in percentage) on $\Delta$ Inventory ${ }^{A}$ and $\Delta$ Distress, with robust t-statistics based on Newey and West (1987) standard errors using the optimal bandwidth choice in Andrews (1991) reported in parentheses. Significance levels are represented by $* \mathrm{p}$ $<0.1{ }^{* *} \mathrm{p}<0.05$, and ${ }^{* * *} \mathrm{p}<0.01$ with $\mathrm{p}$ as the $\mathrm{p}$-value. The last column of Panel $\mathrm{C}$ reports the fraction of the total variation of residuals that is accounted for by the two intermediary factors, denoted as FVE and computed as in (3) for 1-year, 5-year, and 10-year CDS cohorts, as well as all cohorts. The sample period is from 2005:Q1 through 2015:Q2. 
Table 12: Non-Corporate-Credit Assets

\begin{tabular}{|c|c|c|c|c|c|}
\hline \multicolumn{6}{|c|}{ A: Agency MBS } \\
\hline \multirow{3}{*}{$\Delta$ Inventory $^{A}$} & FN30y & FN15y & FG30y & FG15y & \\
\hline & $3.70^{* *}$ & 2.64 & 3.30 & 1.05 & \\
\hline & $(2.10)$ & $(1.58)$ & (1.64) & $(0.55)$ & \\
\hline \multirow[t]{2}{*}{$\Delta$ Distress } & $5.79^{* * *}$ & $6.21^{* * *}$ & $6.55^{* * *}$ & $5.12^{* * *}$ & \\
\hline & $(3.08)$ & $(3.65)$ & $(3.60)$ & $(2.81)$ & \\
\hline \multirow[t]{3}{*}{$R_{a d j}^{2}$} & 0.18 & 0.26 & 0.17 & 0.15 & \\
\hline & & B: CM & & & \\
\hline & Duper & $\mathrm{AM}$ & AJ & & \\
\hline \multirow{2}{*}{$\Delta$ Inventory $^{A}$} & $15.48^{*}$ & 2.94 & 4.33 & & \\
\hline & $(1.89)$ & $(0.16)$ & $(0.21)$ & & \\
\hline \multirow[t]{2}{*}{$\Delta$ Distress } & $28.06^{* * *}$ & $84.50^{* * *}$ & $87.38 * * *$ & & \\
\hline & $(5.10)$ & $(4.24)$ & $(5.14)$ & & \\
\hline \multirow[t]{2}{*}{$R_{a d j}^{2}$} & 0.27 & 0.36 & 0.31 & & \\
\hline & & $\mathrm{C}: \mathrm{AF}$ & & & \\
\hline \multirow{3}{*}{$\Delta$ Inventory $^{A}$} & Credit Card & Auto AAA & Auto A & Auto BBB & \\
\hline & 1.35 & 1.86 & 22.44 & 7.86 & \\
\hline & $(0.40)$ & $(0.44)$ & (1.39) & $(0.34)$ & \\
\hline \multirow[t]{2}{*}{$\Delta$ Distress } & $21.63^{* * *}$ & 6.58 & $145.86^{* * *}$ & $138.35^{* *}$ & \\
\hline & $(4.77)$ & $(1.17)$ & $(3.25)$ & $(2.06)$ & \\
\hline \multirow[t]{3}{*}{$R_{a d j}^{2}$} & 0.37 & 0.02 & 0.51 & 0.39 & \\
\hline & & S\&P 500 in & x options & & \\
\hline & Call: 0.90 & Call: 0.95 & Call: ATM & Call: 1.05 & Call: 1.10 \\
\hline \multirow[t]{2}{*}{$\Delta$ Inventory $^{A}$} & 0.034 & 0.020 & 0.007 & 0.013 & -0.148 \\
\hline & $(0.320)$ & $(0.183)$ & $(0.064)$ & $(0.100)$ & $(-1.101)$ \\
\hline \multirow[t]{2}{*}{$\Delta$ Distress } & 0.263 & 0.257 & 0.314 & 0.272 & 0.225 \\
\hline & $(0.601)$ & $(0.538)$ & $(0.604)$ & $(0.483)$ & $(0.371)$ \\
\hline \multirow[t]{2}{*}{$R_{a d j}^{2}$} & 0.027 & 0.023 & 0.028 & 0.017 & 0.013 \\
\hline & Put: 0.9 & Put: 0.95 & Put: ATM & Put: 1.05 & Put: 1.10 \\
\hline \multirow[t]{2}{*}{$\Delta$ Inventory $^{A}$} & 0.241 & 0.165 & 0.121 & 0.088 & 0.078 \\
\hline & $(0.823)$ & $(0.746)$ & $(0.723)$ & $(0.674)$ & $(0.675)$ \\
\hline \multirow[t]{2}{*}{$\Delta$ Distress } & $0.503^{* * *}$ & $0.355^{* * *}$ & $0.300^{* *}$ & $0.277^{*}$ & 0.227 \\
\hline & $(3.660)$ & $(3.076)$ & $(2.247)$ & $(1.739)$ & $(1.221)$ \\
\hline$R_{a d j}^{2}$ & 0.043 & 0.034 & 0.034 & 0.037 & 0.028 \\
\hline
\end{tabular}

Note: This table reports quarterly time series regressions of residuals of quarterly yield spread changes (in basis points) of agency MBS (in panel A), CMBS (in panel B), and ABS (in panel C) on $\Delta$ Inventory $^{A}$ and $\Delta$ Distress and monthly series regressions of residuals of one-month unannualized returns (in percentage) of $\mathrm{S} \& \mathrm{P} 500$ index option portfolios (in panel D). Each residual series is computed from the regression of the yield spread change or return on the six time series factors included in (1). Robust t-statistics based on Newey and West (1987) standard errors using the optimal bandwidth choice in Andrews (1991) are reported in parentheses, with significance levels indicated by ${ }^{*} \mathrm{p}<0.1,{ }^{* *} \mathrm{p}<0.05$, and ${ }^{* * *} \mathrm{p}<0.01$, where $\mathrm{p}$ is the p-value. The overall sample period is 2005:Q1 - 2015:Q2 for yield spreads, and January 2005 - January 2012 for options. 
Table 13: Average Quarterly Changes of Institutional Investors' Holdings and Dealers' Inventories of Individual Bonds

\begin{tabular}{|c|c|c|c|c|c|c|}
\hline \multicolumn{7}{|c|}{ A: Insurance Companies } \\
\hline & \multicolumn{2}{|c|}{ Downgrade (IG) } & \multicolumn{2}{|c|}{ Fallen Angels } & \multicolumn{2}{|c|}{ No Rating Change } \\
\hline & Obs & mean & Obs & mean & Obs & mean \\
\hline$\Delta$ Holding $_{i, t}$ & 9673 & -0.916 & 3261 & -1.353 & 416254 & -0.390 \\
\hline$\Delta$ Holding $_{i, t+1}$ & 9604 & -1.008 & 3185 & -1.274 & 416965 & -0.404 \\
\hline Holding $_{i, t-1}$ & & 73.359 & & 71.075 & & 87.087 \\
\hline \multicolumn{7}{|c|}{ B: Mutual Funds } \\
\hline & \multicolumn{2}{|c|}{ Downgrade (IG) } & \multicolumn{2}{|c|}{ Fallen Angel } & \multicolumn{2}{|c|}{ No Rating Change } \\
\hline & Obs & mean & Obs & mean & Obs & mean \\
\hline$\Delta$ Holding $_{i, t}$ & 5265 & 0.376 & 1760 & 0.116 & 345154 & -0.423 \\
\hline$\Delta$ Holding $_{i, t+1}$ & 5204 & -0.161 & 1701 & -0.237 & 345385 & -0.390 \\
\hline Holding $_{i, t-1}$ & & 76.882 & & 75.998 & & 65.153 \\
\hline \multicolumn{7}{|c|}{ C: Pension Funds } \\
\hline & \multicolumn{2}{|c|}{ Downgrade (IG) } & \multicolumn{2}{|c|}{ Fallen Angel } & \multicolumn{2}{|c|}{ No Rating Change } \\
\hline & Obs & mean & Obs & mean & Obs & mean \\
\hline$\Delta H_{\text {olding }}, t$ & 4566 & 0.285 & 1484 & 0.204 & 304541 & -0.321 \\
\hline$\Delta$ Holding $_{i, t+1}$ & 4508 & -0.246 & 1443 & -0.474 & 304883 & -0.309 \\
\hline Holding $_{i, t-1}$ & & 19.621 & & 18.110 & & 11.971 \\
\hline \multicolumn{7}{|c|}{ D: Dealers } \\
\hline & \multicolumn{2}{|c|}{ Downgrade (IG) } & \multicolumn{2}{|c|}{ Fallen Angel } & \multicolumn{2}{|c|}{ No Rating Change } \\
\hline & Obs & mean & Obs & mean & Obs & mean \\
\hline$\Delta$ Inventory $_{i, t}$ & 20254 & 0.343 & 6792 & 1.311 & 687927 & 0.254 \\
\hline$\Delta$ Inventor $y_{i, t+1}$ & 18949 & 0.022 & 6449 & -0.275 & 614380 & 0.028 \\
\hline Inventor $_{i, t-1}$ & & 1.949 & & 1.708 & & 1.188 \\
\hline
\end{tabular}

Note: This table reports the average quarterly change of holdings by insurance companies, mutual funds, and pension funds, in panels $\mathrm{A}, \mathrm{B}$, and $\mathrm{C}$, respectively, and the average quarterly change of dealers' inventories in panel $\mathrm{D}$. The average quarterly change for three sets of observations is computed separately: "downgrade (IG)" observations (in the first two columns) with bonds downgraded from IG rating to IG rating, "fallen angels" observations (in the second two columns) with bonds downgraded from IG rating to HY rating, and "no rating change" observations (in the last two columns) with bond experiencing no rating change. Both the number of observations and average amount change (in \$millions) are reported. Both the change in the current quarter and the change in the subsequent quarter are included. For comparison, the average level of institutional holding and dealer inventory (in \$millions) as of the current quarter is reported in the last row of each panel. The sample period is 2005:Q1 - 2015:Q2. 


\section{Table 14: Changes in Institutional Holdings and Dealers' Inventories of Down- graded Bonds}

\begin{tabular}{|c|c|c|c|c|}
\hline \multicolumn{5}{|c|}{ A: Changes of Institutional Holdings and Dealer Inventories in quarter $t$} \\
\hline & Insurance & Mutual & Pension & Dealer \\
\hline & $(1)$ & $(3)$ & $(5)$ & $(7)$ \\
\hline \multirow[t]{2}{*}{ Fallen } & $-0.665^{* * *}$ & -0.219 & -0.058 & $1.607^{* *}$ \\
\hline & $(-3.383)$ & $(-0.574)$ & $(-0.270)$ & $(1.980)$ \\
\hline \multirow[t]{2}{*}{ Downgrade } & $-0.480 * * *$ & $0.509 * *$ & $0.363^{* * *}$ & -0.127 \\
\hline & $(-4.007)$ & $(2.310)$ & $(2.811)$ & $(-0.158)$ \\
\hline Obs & 423,766 & 348,092 & 306,971 & 705,516 \\
\hline $\mathrm{R}_{a d j}^{2}$ & 0.070 & 0.013 & 0.036 & 0.0004 \\
\hline Bond Controls & Yes & Yes & Yes & Yes \\
\hline Time FE & Yes & Yes & Yes & Yes \\
\hline \multicolumn{5}{|c|}{ B: Changes of Institutional Holdings and Dealer Inventories in quarter $t+1$} \\
\hline \multicolumn{2}{|c|}{ Insurance } & Mutual & Pension & Dealer \\
\hline & $(1)$ & $(3)$ & $(5)$ & $(7)$ \\
\hline \multirow[t]{2}{*}{ Fallen } & $-0.326^{*}$ & -0.010 & -0.088 & $-0.447^{* * *}$ \\
\hline & $(-1.654)$ & $(-0.028)$ & $(-0.434)$ & $(-3.187)$ \\
\hline \multirow[t]{2}{*}{ Downgrade } & $-0.795^{* * *}$ & -0.073 & 0.069 & 0.124 \\
\hline & $(-7.125)$ & $(-0.332)$ & $(0.590)$ & $(1.371)$ \\
\hline Obs & 424,413 & 348,266 & 307,265 & 630,957 \\
\hline $\mathrm{R}_{a d j}^{2}$ & 0.071 & 0.013 & 0.036 & 0.001 \\
\hline Bond Controls & Yes & Yes & Yes & Yes \\
\hline Time FE & Yes & Yes & Yes & Yes \\
\hline
\end{tabular}

Note: The first three columns report panel regressions in (17) of changes in institutional holdings of bond $i$ in quarter $t+\tau$ ( $\tau$ equals 0 in in panel $\mathrm{A}$ and 1 in panel $\mathrm{B}$ ) on indicator variables Downgrade $_{i, t}$, which equals 1 if bond $i$ is downgraded from IG rating to either IG or HY rating in quarter $t$ and 0 otherwise and indicator Fallen $n_{i, t}$ that equals 1 if bond $i$ is downgraded from IG rating to HY rating in quarter $t$ and 0 otherwise, for insurance companies, mutual funds, and pension funds, respectively. Similar panel regressions of changes in dealers' inventories $\Delta$ Inventor $y_{i, t+\tau}$ are reported in the last column. Bond controls include the log of outstanding balance in \$thousands $\left(\log \left(\mathrm{Amt}_{i, t+\tau}\right)\right)$, the $\log$ of issue size in $\$$ millions $\left(\log \left(\mathrm{Size}_{i}\right)\right)$, bond age in years $\left(\mathrm{Age}_{i, t+\tau}\right)$, and time-to-maturity in years (Time-to-Mature $i_{i, t+\tau}$ ). For simplicity, we suppress the coefficients on these controls and the intercept. The sample includes observations of bonds downgraded from investment grade to either investment grade or high yield and of bonds with no rating change. Robust t-statistics based on clustered standard errors at the bond level are reported in parentheses with significance levels represented by $*$ for $\mathrm{p}<0.1,{ }^{* *}$ for $\mathrm{p}<0.05$, and ${ }^{* * *}$ for $\mathrm{p}<0.01$, where $\mathrm{p}$ is the p-value. The sample period is from 2005:Q1 - 2015:Q2. 


\section{Table 15: First-Stage Regressions}

\begin{tabular}{|c|c|c|c|}
\hline & $\Delta$ Inventory $y_{t}^{A}$ & $\Delta$ Inventory $y_{t}^{A}$ & $\Delta$ Inventory $y_{t}^{A}$ \\
\hline \multirow{2}{*}{$\Delta$ Holding $_{t}^{F A}$} & $-0.377^{* * *}$ & & $-0.369^{* * *}$ \\
\hline & $(-2.618)$ & & $(-3.983)$ \\
\hline \multirow[t]{2}{*}{ Insurance $\operatorname{Loss}_{t}$} & & 0.101 & 0.073 \\
\hline & & $(1.188)$ & $(1.413)$ \\
\hline \multirow[t]{2}{*}{$\Delta$ Distress } & $0.552^{* * *}$ & $0.456^{* * *}$ & $0.545^{* * *}$ \\
\hline & $(4.890)$ & $(3.423)$ & $(5.738)$ \\
\hline \multirow[t]{2}{*}{$\Delta$ Holding ${ }_{t}^{D}$} & 0.045 & $-0.179^{*}$ & 0.047 \\
\hline & $(0.255)$ & $(-1.701)$ & $(0.273)$ \\
\hline \multirow[t]{2}{*}{$\Delta V I X$} & 0.003 & 0.005 & 0.002 \\
\hline & $(0.114)$ & $(0.176)$ & $(0.146)$ \\
\hline \multirow[t]{2}{*}{$\Delta J u m p$} & $-15.995^{* * *}$ & $-13.876^{* *}$ & $-15.775^{* * *}$ \\
\hline & $(-3.260)$ & $(-2.156)$ & $(-6.003)$ \\
\hline \multirow[t]{2}{*}{$\Delta r^{10 y}$} & $0.806^{*}$ & 0.654 & $0.728^{* * *}$ \\
\hline & $(1.934)$ & $(1.547)$ & $(4.242)$ \\
\hline \multirow[t]{2}{*}{$\left(\Delta r^{10 y}\right)^{2}$} & -0.294 & -0.396 & -0.314 \\
\hline & $(-1.043)$ & $(-1.270)$ & $(-1.014)$ \\
\hline \multirow[t]{2}{*}{$\Delta$ Slope } & -0.400 & -0.359 & $-0.352^{* *}$ \\
\hline & $(-1.158)$ & $(-0.909)$ & $(-2.302)$ \\
\hline \multirow[t]{2}{*}{$\operatorname{Ret}_{t}^{S P}$} & $7.591^{* * *}$ & $7.088^{* * *}$ & $7.842^{* * *}$ \\
\hline & $(4.462)$ & $(3.194)$ & $(9.014)$ \\
\hline \multirow[t]{2}{*}{ Intercept } & 0.041 & 0.066 & 0.040 \\
\hline & $(0.258)$ & $(0.417)$ & $(0.324)$ \\
\hline $\mathbf{R}_{a d j}^{2}$ & 0.547 & 0.482 & 0.552 \\
\hline
\end{tabular}

Note: This table reports the first-stage regressions of $\Delta$ Inventory $^{A}$ on $\Delta$ Holding $_{t}^{F A}$ and $\operatorname{InsuredLoss}_{t}$, separately in the first two columns and jointly in the third column. The change in institutional holdings of all downgraded bonds $\Delta$ Holding ${ }_{t}^{D}$ is included as a control, in addition to $\Delta$ Distress and the six time series variables used in the baseline bond-level regression (1). All measures except the six time series variable from (1) are scaled to have zero mean and unit variance. Robust t-statistics based on Newey and West (1987) standard errors using the optimal bandwidth choice in Andrews (1991) are reported in parentheses, with significance levels indicated by ${ }^{*} \mathrm{p}<0.1,{ }^{* *} \mathrm{p}<0.05$, and ${ }^{* * *} \mathrm{p}<0.01$, where $\mathrm{p}$ is the $\mathrm{p}$-value. The sample period is 2005:Q1 - 2015:Q2. 
Table 16: Second-Stage Regressions

\begin{tabular}{|c|c|c|c|c|c|c|c|c|}
\hline \multicolumn{2}{|c|}{ Groups } & \multicolumn{2}{|c|}{ A: $\Delta H o l d i n g_{t}^{F A}$} & \multicolumn{3}{|c|}{ B: Insurance Losst } & \multicolumn{2}{|c|}{ C: $\Delta$ Holding $_{t}^{F A}+$ Insurance Loss $t$} \\
\hline Maturity & Rating & $\Delta$ Inventory $y_{t}^{A}$ & $\Delta$ Distress $s_{t}$ & $\Delta \operatorname{Inv}$ & ory $y_{t}^{A}$ & $\Delta$ Distress $_{t}$ & $\Delta$ Inventory $y_{t}^{A}$ & $\Delta$ Distress $_{t}$ \\
\hline \multirow[t]{2}{*}{ Short } & \multirow[t]{2}{*}{ AA } & $0.208^{* *}$ & 0.004 & \multicolumn{2}{|c|}{0.188} & 0.014 & $0.206^{* * *}$ & 0.005 \\
\hline & & $(2.375)$ & $(0.057)$ & {$[0.254]$} & {$[0.164]$} & $(0.177)$ & $(2.945)$ & $(0.090)$ \\
\hline \multirow[t]{2}{*}{ Short } & \multirow[t]{2}{*}{ A } & $0.208^{*}$ & 0.081 & \multicolumn{2}{|c|}{0.214} & 0.078 & $0.208^{* *}$ & 0.081 \\
\hline & & $(1.828)$ & $(1.015)$ & {$[0.074]$} & {$[0.157]$} & $(1.041)$ & $(2.459)$ & $(1.383)$ \\
\hline Short & BBB & 0.188 & $0.199 * * *$ & \multicolumn{2}{|c|}{0.191} & $0.197 * * *$ & $0.188^{* *}$ & $0.198^{* * *}$ \\
\hline Short & $\mathrm{BB}$ & $(1.679)$ & $(1.762)$ & {$[0.070]$} & {$[0.145]$} & $(0.916)$ & $(2.541)$ & $(2.262)$ \\
\hline \multirow[t]{2}{*}{ Short } & \multirow[t]{2}{*}{ B } & $0.740^{* *}$ & $0.630^{* *}$ & \multicolumn{2}{|c|}{1.463} & 0.270 & $0.818^{* * *}$ & $0.591 * * *$ \\
\hline & & $(2.181)$ & $(2.448)$ & {$[0.0004$} & [0.135] & $(0.666)$ & $(3.822)$ & $(3.491)$ \\
\hline \multirow[t]{2}{*}{ Medium } & \multirow[t]{2}{*}{$\mathrm{AA}$} & $0.208^{* *}$ & -0.003 & \multicolumn{2}{|c|}{0.140} & 0.031 & $0.201^{* *}$ & 0.001 \\
\hline & & $(1.973)$ & $(-0.038)$ & [0.265] & {$[0.157]$} & $(0.603)$ & $(2.249)$ & $(0.012)$ \\
\hline Medium & BBB & $(1.873)$ & $(2.994)$ & {$[0.026]$} & {$[0.146]$} & $(2.960)$ & $(2.345)$ & $(3.762)$ \\
\hline Medium & $\mathrm{BB}$ & $0.544^{*}$ & $0.294^{*}$ & & & $0.285^{* *}$ & $0.546^{* *}$ & $0.293^{* *}$ \\
\hline & & $(1.792)$ & $(1.726)$ & {$[0.037]$} & {$[0.137]$} & $(1.993)$ & $(2.428)$ & $(2.495)$ \\
\hline Medium & B & $0.671^{* * *}$ & $0.668^{* * *}$ & & & $0.773^{* * *}$ & $0.648^{* * *}$ & $0.679 * * *$ \\
\hline & & $(2.621)$ & $(4.116)$ & [0.023] & [0.174] & $(4.419)$ & $(3.492)$ & $(6.637)$ \\
\hline Long & AA & $0.123^{* * *}$ & 0.012 & & & 0.088 & $0.106^{* *}$ & 0.020 \\
\hline & & $(2.784)$ & $(0.278)$ & {$[0.757]$} & {$[0.642]$} & $(1.553)$ & $(2.553)$ & $(0.542)$ \\
\hline Long & $\mathrm{A}$ & $0.214^{* *}$ & 0.035 & & & $0.113^{* *}$ & $0.197^{* *}$ & 0.043 \\
\hline & & $(2.225)$ & $(0.472)$ & [0.533] & {$[0.383]$} & $(2.432)$ & $(2.426)$ & $(0.697)$ \\
\hline Long & BBB & 0.144 & 0.213 & & & $0.215^{*}$ & 0.144 & $0.213^{*}$ \\
\hline & & $(0.848)$ & $(1.214)$ & [0.598] & [0.295] & $(1.886)$ & $(1.345)$ & $(1.807)$ \\
\hline
\end{tabular}

Note: This table reports second-stage regressions of quarterly residuals of quarterly credit spread changes (in percentage) on $\Delta$ Distress and $\Delta$ Inventory $^{A}$, using $\Delta$ Holding $_{t}^{F A}$ as instrument in panel A, InsuredLoss $t_{t}$ as instrument in panel $\mathrm{B}$, and both as instruments in panel C. Regression coefficients on $\Delta$ Inventory ${ }^{A}$ and $\Delta$ Distress are reported, but those on control variables $\left(\Delta\right.$ Holding $_{t}^{D}, \Delta$ Distress, and the six time series variables used in (1)) are omitted for simplicity of reporting. The last two rows in each panel report the test statistic for weak instruments by Montiel-Olea and Pflueger (2013) (MP) and associated critical values (Pflueger and Wang (2015)). An MP-statistic greater than critical values in brackets below rejects the hypothesis of weak instruments (with a worst-case bias greater than $20 \%$ of the OLS bias) at a significance level of $10 \%$. Robust t-statistics based on Newey and West (1987) standard errors using the optimal bandwidth choice in Andrews (1991) are reported in parentheses, with significance levels indicated by ${ }^{*} \mathrm{p}<0.1,{ }^{* *} \mathrm{p}$ $<0.05$, and ${ }^{* * *} \mathrm{p}<0.01$, where $\mathrm{p}$ is the $\mathrm{p}$-value. For the coefficient on $\Delta$ Inventory $^{A}$ in panel $\mathrm{B}$, $\mathrm{p}$-values of the Anderson and Rubin (1949) Wald-test and the Stock and Wright (2000) S-statistic are reported in the left and right brackets, which are both weak-instrument robust for testing the significance of $\Delta$ Inventory $y_{t}^{A}$. The sample period is from 2005:Q1 - 2015:Q2. 


\section{Table 17: Regressions of Bond-Return Factors on Intermediary Factors}

\begin{tabular}{|c|c|c|c|c|}
\hline & $\mathrm{MKT}^{\text {Bond }}$ & DRF & $\mathrm{CRF}$ & LRF \\
\hline \multicolumn{5}{|c|}{ A: Regressions on Dealer Inventory } \\
\hline \multirow[t]{2}{*}{$\Delta$ Inventory $y_{t}^{A}$} & 0.027 & -0.008 & 0.111 & -0.149 \\
\hline & $(0.280)$ & $(-0.038)$ & $(0.508)$ & $(-0.812)$ \\
\hline $\mathrm{R}_{a d j}^{2}$ & 0.002 & 0.000 & 0.009 & 0.017 \\
\hline \multicolumn{5}{|c|}{ B: Regressions on Intermediary Distress } \\
\hline \multirow[t]{2}{*}{$\Delta$ Distress $_{t}$} & $-0.388^{* * *}$ & $-0.941^{* * *}$ & $-0.651^{* * *}$ & $-1.120^{* * *}$ \\
\hline & $(-2.807)$ & $(-5.201)$ & $(-3.635)$ & $(-6.280)$ \\
\hline $\mathrm{R}_{a d j}^{2}$ & 0.235 & 0.324 & 0.202 & 0.586 \\
\hline \multicolumn{5}{|c|}{ C: Regressions on Dealer Inventory and Intermediary Distress } \\
\hline \multirow[t]{2}{*}{$\Delta$ Inventoryt ${ }_{t}^{A}$} & 0.103 & 0.173 & 0.242 & 0.058 \\
\hline & $(1.341)$ & $(1.064)$ & $(1.304)$ & $(0.485)$ \\
\hline \multirow[t]{2}{*}{$\Delta$ Distress $_{t}$} & $-0.418^{* * *}$ & $-0.995^{* * *}$ & $-0.721^{* * *}$ & $-1.136^{* * *}$ \\
\hline & $(-3.231)$ & $(-5.558)$ & $(-4.287)$ & $(-6.566)$ \\
\hline $\mathrm{R}_{a d j}^{2}$ & 0.263 & 0.367 & 0.244 & 0.593 \\
\hline
\end{tabular}

Note: This table reports quarterly time series regressions of return-based factors, including corporate bond market return $\left(\mathrm{MKT}^{\text {Bond }}\right)$, downside risk factor (DRF), credit risk factor $(\mathrm{CRF})$, and liquidity risk factor (LRF) of Bai, Bali, and Wen (2019), on $\Delta$ Inventory $^{A}$ and $\Delta$ Distress. The original series of return factors are one-month returns (in percent) of monthly rebalanced portfolios, and we construct quarterly return factors using geometric mean of the three monthly returns for each quarter. We orthogonalize both the return factors and intermediary factors against the six time series structural factors as used in (1). Robust t-statistics based on Newey and West (1987) standard errors using the optimal bandwidth choice in Andrews (1991) are reported in parentheses. Significance levels are represented by $* \mathrm{p}<0.1,{ }^{* *} \mathrm{p}<0.05$, and ${ }^{* * *} \mathrm{p}$ $<0.01$ with $\mathrm{p}$ as the p-value. The sample period is from 2005:Q1 through 2015:Q2. 


\section{Appendices}

\section{A Additional Data Summary and Empirical Results}

In this appendix, we provide additional data summary statistics and empirical results.

First, Table A.1 reports the detailed procedure of sample cleaning and construction.

Second, Figure A.1 and Table A.2 provide a summary of the eMAXX institutional holdings. The top panel of Figure A.1 shows the quarterly series of the total number of institutions, which increased from about 5000 to more than 6000. This increase is mainly due to the growth of mutual funds, whereas the number of insurance companies remains stable around 2800. As shown in the middle panel, the total number of bonds held by these institutions is about 15000 steadily, and largest by insurance companies. Finally, the bottom panel plots quarterly series of the total holding amount by all institutions and outstanding balance of an average bond, calculated as the respective average of the total holding amount and outstanding balance across all bonds in each quarter. The average holding amount and outstanding have increased roughly in parallel to each other, so the institutional holding steadily accounts for 30-35\% of the outstanding except a brief drop during the 2008 crisis.

Panel A of Table A.2 reports the number of institutional investors, panel B reports the number of bonds, and panel $\mathrm{C}$ reports the aggregate holding amount in principal value, by insurance companies, mutual funds, pension funds, and all institutions separately. Panel D reports summary statistics of quarterly series of the total holding amount by all institutions and the outstanding balance, of an average bond. Specifically, for each bond in each quarter, we first sum the holding amounts by all institutions to obtain a total holding amount Holding $_{i t}$. Then across all the bonds $i$ in each quarter, we compute the mean of Holding $g_{i t}$ as the total holding amount of an average bond (or average bond's holding amount). Across all the bonds in each quarter, we also compute the mean of outstanding balance as the outstanding balance of an average bond (or average bond's outstanding balance). In each quarter, we compute the ratio of average holding amount to average outstanding balance and obtain a quarter series of average holding/outstanding.

Third, Table A.3 reports summary statistics of quarterly time series of option-adjusted spreads of agency MBS, yield spreads of non-agency CMBS, and yield spreads of ABS all in basis points, in panels A, B, and C, respectively. Panel D reports summary statistics of monthly time series of (unannualized) one-month return of leverage-adjusted S\&P 500 index option portfolios in percentage.

Fourth, panel A of of Table A.4 reports quarterly time series regressions of the baseline residuals on the two intermediary factors, with $\Delta$ Inventory $^{A}$ based on dollar value of corporate bond transactions, as opposed to par value used in the baseline measure.

Fifth, in the baseline analysis, $\Delta$ Inventory ${ }^{A}$ and $\Delta$ Distress are both measured using changes between two quarter ends. In contrast, the credit spread change $\Delta c s$ may not be exactly between two quarter ends, and the time duration of the change ranges from 45 to 120 days. Panel B of Table A.4 reports quarterly time series regressions of the baseline residuals on intermediary factors that are constructed by matching to the horizon of credit spread changes. Specifically, for each observation $\Delta c s_{i t}$, we compute measures $\Delta$ Inventory $y_{i t}^{\text {match }}$ and 
$\Delta$ Distress match $^{\text {at }}$ as the changes of dealer inventory and intermediary distress measures over the same time horizon. We then take the average of $\Delta$ Inventor $y_{i t}^{\text {match }}$ and $\Delta$ Distress ${ }_{i t}^{\text {match }}$ across all bonds in each quarter $t$ as the aggregate time series measures of intermediary factors, denoted as $\Delta$ Inventory $y_{t}^{\text {match }}$ and $\Delta$ Distress $t_{t}^{\text {match }}$. That is, these alternative measures take into account the distribution of time horizons of credit spread changes across bonds.

Sixth, Table A.5 reports quarterly time series regressions of baseline residuals on baseline intermediary factors, controlling for two other potential measures of intermediary distress, the leverage measure of broker-dealers in Adrian, Etula, and Muir (2014), here constructed in the same nonlinear way as in our baseline HKM measure, i.e., $\Delta \mathrm{NLev}_{t}^{\mathrm{AEM}}:=\left(\mathrm{Lev}_{t}^{\mathrm{AEM}}-\right.$ $\left.\operatorname{Lev}_{t-1}^{\mathrm{AEM}}\right) \times \operatorname{Lev}_{t-1}^{\mathrm{AEM}}$ (in panel A) and TED spread computed as the difference between threemonth Libor and T-bill rates (in panel B). We find that the broker-dealer leverage does not have incremental explanatory power relative to our two intermediary factors. TED spread adds certain explanatory power, statistically significant for IG bonds with similar economic significance for different cohorts, different from the monotonic increasing effect of our two intermediary factors with decreasing ratings.

Seventh, Table A.6 reports quarterly time series regressions of baseline residuals on intermediary factors, controlling for the Pástor and Stambaugh (2003) stock liquidity factor (in panel A), and monthly time series regressions controlling for the Bao, Pan, and Wang (2011) corporate bond liquidity factor (in panel B). We find that neither of these two liquidity factors contribute significant incremental explanatory power in explaining common credit spread changes.

Eighth, one may be concerned that the strong explanatory power documented is mainly due to the inclusion of the 2008 financial crisis. Table A.7 reports results following the baseline procedure but excluding the 2008 financial crisis period (defined as 2007:Q3 - 2009:Q1 similar to Bao, O'Hara, and Zhou (2018), Schultz (2017), and others). From Panel A of the $\mathrm{PC}$ analysis, we observe a strong common variation with the $\mathrm{PC} 1$ accounting for $80 \%$ of the total unexplained variation of credit spread changes. From Panel B of the quarterly bivariate series regressions of on dealer inventory and intermediary distress, intermediary factors have significant positive effects that monotonically increase with decreasing ratings, and similar economic significance. The two factors together account for $48 \%$ of the unexplained total variation of credit spread changes, slightly higher than that in the baseline Table 4 including the crisis observations.

Ninth, Table A.8 reports time series correlations of the three different inventory measures, $\Delta$ Inventory $^{A}, \Delta$ Inventory $^{H Y}$, and $\Delta$ Inventory $^{I G}$. We consider both simple changes and percentage changes. We observe that $\Delta$ Inventory ${ }^{A}$ is positively correlated with both $\Delta$ Inventory ${ }^{H Y}$ and $\Delta$ Inventory ${ }^{I G}$ at a $10 \%$ significance level. Importantly, the correlation between $\Delta$ Inventory ${ }^{H Y}$ and $\Delta$ Inventory ${ }^{I G}$ is slightly negative in raw changes and near zero in percentage changes, statistically insignificant.

Finally, Table A.9 reports summary statistics of corporate bond holdings of insurance companies, mutual funds, and pension funds by rating groups. We find that insurance companies have a lower fraction of holdings in HY bonds than mutual funds and pension funds, consistent with strict regulatory constraints on insurance companies (Ellul, Jotikasthira, and Lundblad (2011)). 


\section{B Model Extensions}

\section{B.1 More Traditional Margin Constraints}

The form of our margin-like constraint,

$$
\sum_{a=1}^{A} \theta_{I, a} m_{a} \leq w
$$

is chosen for analytical tractability but differs from reality in two basic ways. First, margin is typically required for both long and short positions. Such a constraint, similar to Garleanu and Pedersen (2011), would be

$$
\sum_{a=1}^{A}\left|\theta_{I, a}\right| m_{a} \leq w .
$$

Constraint (19) will deliver the additional prediction that the law of one price can fail. Two assets with the same payoffs but different margin requirements can be priced differently, which can be used to discuss empirical phenomena such as the bond-CDS basis or coveredinterest-parity deviations. Our empirics do not focus on such situations. Furthermore, since our model focuses on hedgers' demand for insurance (through $h>0$ ), intermediaries will typically hold long positions $\left(\theta_{I}>0\right)$, making (19) equivalent to (18).

Second, margin requirements $m$ typically depend on current and future asset prices. For example, if margin is calculated as a fraction of the expenditure on assets, then $m_{a}=\bar{m}_{a} p_{a}$ in (18), i.e.,

$$
\sum_{a=1}^{A} \theta_{I, a} \bar{m}_{a} p_{a} \leq w .
$$

Constraints augmented with price, as in (20), will have an additional mitigating force to (18). Indeed, a positive $s$-shock decreases asset prices and thus loosens constraint (20) through lower margin requirements. Equilibrium prices fall by less than they would under (18). As another example, exchanges often compute margin based on future prices, through return volatility, in which case $m_{a}=\bar{m}_{a} p_{a} v_{a}$. As prices and volatilities tend to be negatively correlated, this formulation would tend to amplify our effects: a price decline accompanied by a volatility spike would tighten the margin constraint. Since these forces are qualitatively similar to our baseline model, just mitigated or amplified, we ignore them and focus on (18).

\section{B.2 Asset-Class-Specific Constraints}

Rather than a single margin constraint, suppose dealers face asset-class-specific margin constraints. For example, different trading desks within a bank may be given independent portfolio limits. Alternatively, there may be some market segmentation - different intermediaries, each having its own margin constraint, may participate in non-overlapping asset markets. Mathematically, partition the assets $a \in\{1, \ldots, A\}$ into two subsets $\mathcal{A}_{1}$ and $\mathcal{A}_{2}$, 
and impose different constraints on the subsets:

$$
\sum_{a \in \mathcal{A}_{1}} \theta_{I, a} m_{a} \leq w_{1} \quad \text { and } \quad \sum_{a \in \mathcal{A}_{2}} \theta_{I, a} m_{a} \leq w_{2}
$$

If we interpret (21) as desk-specific constraints within a given intermediary, we would set $w_{1}=w_{2}=w$. Otherwise, there are two types of intermediaries, with wealths $w_{1}$ and $w_{2}$ that sum to aggregate intermediary wealth $w$. Under (21), there are two Lagrange multipliers, $\mu_{1}$ and $\mu_{2}$ associated with each inequality. The pricing condition (8) is modified to be $p=\bar{\delta}-\mathbf{1}_{A}-\operatorname{diag}(m)\left(\mu_{1} \mathbf{1}_{\mathcal{A}_{1}}+\mu_{2} \mathbf{1}_{\mathcal{A}_{2}}\right)$. In this case, shocks affecting one of the Lagrange multipliers disproportionately more than the other, such as asset-specific supply shocks, will have an outsized effect on those assets.

Fully solving the model, one can derive the results of the following Lemma.

Lemma 1. The Lagrange multipliers are given by

$$
\begin{aligned}
& \mu_{1}= \begin{cases}\alpha\left[\mathbf{1}_{\mathcal{A}_{1}}^{\prime} M \Sigma^{-1} M \mathbf{1}_{\mathcal{A}_{1}}\right]^{-1} x_{1}, & \text { if } x_{1} \geq 0, x_{2}<\phi_{1} x_{1} \\
\alpha\left[\mathbf{1}_{\mathcal{A}_{1}}^{\prime} M \Sigma^{-1} M \mathbf{1}_{\mathcal{A}_{1}}\right]^{-1}\left[1-\phi_{1} \phi_{2}\right]^{-1}\left[x_{1}-\phi_{2}\left(x_{2}\right)^{+}\right]^{+}, & \text {otherwise }\end{cases} \\
& \mu_{2}= \begin{cases}\alpha\left[\mathbf{1}_{\mathcal{A}_{2}}^{\prime} M \Sigma^{-1} M \mathbf{1}_{\mathcal{A}_{2}}\right]^{-1} x_{2}, & \text { if } x_{2} \geq 0, x_{1}<\phi_{2} x_{2} \\
\alpha\left[\mathbf{1}_{\mathcal{A}_{2}}^{\prime} M \Sigma^{-1} M \mathbf{1}_{\mathcal{A}_{2}}\right]^{-1}\left[1-\phi_{1} \phi_{2}\right]^{-1}\left[x_{2}-\phi_{1}\left(x_{1}\right)^{+}\right]^{+}, & \text {otherwise }\end{cases}
\end{aligned}
$$

where $M:=\operatorname{diag}(m)$, and where

$$
\begin{aligned}
\phi_{1} & :=\frac{\mathbf{1}_{\mathcal{A}_{1}}^{\prime} M \Sigma^{-1} M \mathbf{1}_{\mathcal{A}_{2}}}{\mathbf{1}_{\mathcal{A}_{1}}^{\prime} M \Sigma^{-1} M \mathbf{1}_{\mathcal{A}_{1}}} \quad \text { and } & \phi_{2}:=\frac{\mathbf{1}_{\mathcal{A}_{1}}^{\prime} M \Sigma^{-1} M \mathbf{1}_{\mathcal{A}_{2}}}{\mathbf{1}_{\mathcal{A}_{2}}^{\prime} M \Sigma^{-1} M \mathbf{1}_{\mathcal{A}_{2}}} \\
x_{1} & :=h^{\prime} M \mathbf{1}_{\mathcal{A}_{1}}-w_{1} \quad \text { and } & x_{2}:=h^{\prime} M \mathbf{1}_{\mathcal{A}_{2}}-w_{2}
\end{aligned}
$$

With Lemma 1, we can study how prices respond to different shocks. Suppose $\mathcal{A}_{1}$ are corporate bonds and $\mathcal{A}_{2}$ are other assets. Write $h=s_{1} \bar{h}_{1}+s_{2} \bar{h}_{2}$ for scalars $s_{1}, s_{2}$ and vectors $\bar{h}_{1}, \bar{h}_{2}$ that are independent, i.e., $\bar{h}_{1} \cdot \bar{h}_{2}=0$. Then, it is easy to see that $x_{1}=s_{1} \bar{h}_{1}^{\prime} M \mathbf{1}_{\mathcal{A}_{1}}-w_{1}$ and $x_{2}=s_{2} \bar{h}_{2}^{\prime} M \mathbf{1}_{\mathcal{A}_{2}}-w_{2}$. A supply shock to $s_{1}$ is a pure shock to $x_{1}$. When supply of bonds is sufficiently high such that $x_{1}>0$, then $\mu_{1}>0$, and $\mu_{1}$ is strictly increasing in $s_{1}$, whereas $\mu_{2}$ is invariant to $s_{1}{ }^{36}$ In words, pure bond supply shocks only affect prices of bonds and other assets traded on the bond desk, i.e., assets in $\mathcal{A}_{1}$.

On the other hand, shocks affecting aggregate intermediary wealth $w$ affect all assets in a similar manner to the baseline model. Consider a shock to $w_{1}+w_{2}=w$ such that $w_{1} / w_{2}$

\footnotetext{
${ }^{36}$ Lemma 1 also shows that it is possible to have $\mu_{1}$ increasing in $s_{1}$, while $\mu_{2}$ is decreasing in $s_{1}$. This occurs when $x_{1}, x_{2}<0, x_{2}<\phi_{1} x_{1}$, and $x_{1}<\phi_{2} x_{2}$. This case helps explain why non-bond assets may be insensitive to bond inventory even if trading desks are sometimes integrated. Indeed, integrated trading desks implies all prices are sensitive to bond inventory, as in Proposition 2. In contrast, segmented trading desks implies there is a region in which non-bond assets are completely insensitive to bond inventory, and a region in which non-bond assets are oppositely sensitive to bond inventory. The existence of these three regions with differing sensitivities thus muddies the observed empirical relationships between non-bond assets and bond inventory.
} 
remains constant. Both $x_{1}$ and $x_{2}$ unambiguously rise, which one can show weakly increases $\mu_{1}$ and $\mu_{2}$. The result is a larger price discount, induced by tightening constraints on all trading desks.

\section{B.3 Risk-Averse Intermediaries}

Here, we generalize the model by assuming intermediaries have mean-variance preferences with risk aversion $\gamma(w)$, an exogenously decreasing function of $w$. The dependence of risk aversion on wealth $w$ captures the wealth-effect mechanism of Kyle and Xiong (2001) and others. The benchmark results are obtained in the appropriate limit $\gamma \rightarrow 0$. Specifically, suppose intermediaries solve

$$
\begin{aligned}
& \max _{\theta_{I}} \mathbb{E}\left[W_{I}\right]-\frac{\gamma(w)}{2} \operatorname{Var}\left[W_{I}\right] \\
& \text { s.t. } \quad W_{I}:=w+\theta_{I} \cdot\left(\delta-p-\mathbf{1}_{A}\right) \quad \text { and } \quad \theta_{I} \cdot m \leq w .
\end{aligned}
$$

Letting $\mu$ denote the Lagrange multiplier on the margin constraint, the optimal intermediary portfolio is given by

$$
\theta_{I}=(\gamma(w) \Sigma)^{-1}\left[\bar{\delta}-p-\mathbf{1}_{A}-\mu m\right]
$$

Clearing markets with (4), asset prices satisfy

$$
\bar{\delta}-p-\mathbf{1}_{A}=\Gamma(w)\left[\Sigma h+\gamma(w)^{-1} \mu m\right]
$$

where $\Gamma(w):=\left(\alpha^{-1}+\gamma(w)^{-1}\right)^{-1}$. Combining these results with the margin constraint, we have that

$$
\mu=\frac{\alpha}{m^{\prime} \Sigma^{-1} m}\left[m^{\prime} h-\left(1+\frac{\gamma(w)}{\alpha}\right) w\right]^{+}
$$

which can be plugged into the expression for prices to solve completely for equilibrium.

Proposition 3. If the intermediary margin constraint is binding, i.e., $w<w^{*}:=\{\tilde{w}: \tilde{w}=$ $\left.\left(1+\frac{\gamma(\tilde{w})}{\alpha}\right)^{-1} m^{\prime} h\right\}$, then

$$
\begin{aligned}
& \text { ("Supply Shock") } \frac{\partial p}{\partial s}=-\Gamma(w) \Sigma \bar{h}_{\text {bond }}+\frac{\Gamma(w)}{\gamma(w)}\left(\frac{m^{\prime} \bar{h}_{\text {bond }}}{m^{\prime} \Sigma^{-1} m}\right) \alpha m \\
& \text { ("Demand Shock") } \frac{\partial p}{\partial w}=\frac{\Gamma(w)^{2}}{w \gamma(w)} \epsilon_{\gamma}(w) \Sigma h \\
& +\left[1-\frac{1}{w}\left(\frac{\gamma(w)-1}{\gamma(w)} \Gamma(w) w-(\Gamma(w)-1) m^{\prime} h\right) \frac{\Gamma(w)}{\gamma(w)} \epsilon_{\gamma}(w)\right] \frac{\alpha m}{m^{\prime} \Sigma^{-1} m} .
\end{aligned}
$$


where $\epsilon_{\gamma}(w):=-\frac{w \gamma^{\prime}(w)}{\gamma(w)}>0$ is the risk-aversion-wealth elasticity. Otherwise, if $w>w^{*}$, then

$$
\begin{aligned}
\text { ("Supply Shock") } \frac{\partial p}{\partial s} & =-\Gamma(w) \Sigma \bar{h}_{\text {bond }} \\
\text { ("Demand Shock") } & \frac{\partial p}{\partial w}=\frac{\Gamma(w)^{2}}{w \gamma(w)} \epsilon_{\gamma}(w) \Sigma h .
\end{aligned}
$$

Proposition 3 can help explain why some asset classes may display trivial sensitivity to bond supply $s$ (and its empirical proxy, bond inventory $\xi$ ) even when they display large sensitivity to intermediary wealth $w$ (and its empirical proxy, leverage $\lambda$ ). These will be assets with low margin requirements and low covariance to bonds. For example, consider an asset $a$ which has $m_{a}=0$ and zero fundamental correlation to any other asset, including bonds. In that case, $\partial p_{a} / \partial s=0$ whereas $\partial p_{a} / \partial w=\frac{\Gamma(w)^{2}}{w \gamma(w)} \epsilon_{\gamma}(w) \sigma_{a}^{2} h_{a}>0$ (these formulas hold independent of whether the margin constraint binds). Notice that the discrepancy between $\partial p_{a} / \partial s$ and $\partial p_{a} / \partial w$ is increasing in the asset's own volatility $\sigma_{a}$ and hedger's liquidity demand $h_{a}$. 


\section{References}

Acharya, V. V., Amihud, Y., and Bharath, S. T. 2013. Liquidity risk of corporate bond returns: conditional approach. Journal of Financial Economics 110: 358 - 386.

Adrian, T., Etula, E., and Muir, T. 2014. Financial Intermediaries and the Cross-Section of Asset Returns. The Journal of Finance 69: 2557-2596.

Ambrose, B. W., Cai, N. K., and Helwege, J. 2008. Forced Selling of Fallen Angels. The Journal of Fixed Income 18: 72-85.

Amihud, Y. 2002. Illiquidity and stock returns: cross-section and time-series effects. Journal of Financial Markets 5: 31-56.

Anand, A., Jotikasthira, C., and Venkataraman, K. 2018. Do Buy-Side Institutions Supply Liquidity in Bond Markets? Evidence from Mutual Funds. Working Paper.

Anderson, T. W., and Rubin, H. 1949. Estimation of the Parameters of a Single Equation in a Complete System of Stochastic Equations. The Annals of Mathematical Statistics 20: 46-63.

Andrews, D. 1991. Heteroskedasticity and Autocorrelation Consistent Covariance Matrix Estimation. Econometrica 59: 817-858.

Bai, J., Bali, T. G., and Wen, Q. 2019. Common risk factors in the cross-section of corporate bond returns. Journal of Financial Economics 131: 619 - 642.

Bank for International Settlements 2016. Electronic trading in fixed income markets. Report submitted by a Study Group established by the Markets Committee and chaired by Joachim Nagel (Deutsche Bundesbank).

Bao, J., and Hou, K. 2017. De Facto Seniority, Credit Risk, and Corporate Bond Prices. The Review of Financial Studies 30 (08): 4038-4080.

Bao, J., O'Hara, M., and Zhou, X. A. 2018. The Volcker Rule and corporate bond market making in times of stress. Journal of Financial Economics 130: 95 - 113.

Bao, J., and Pan, J. 2013. Bond Illiquidity and Excess Volatility. The Review of Financial Studies 26 (07): 3068-3103.

Bao, J., Pan, J., and Wang, J. 2011. The Illiquidity of Corporate Bonds. The Journal of Finance 66: 911-946.

Bessembinder, H., Jacobsen, S., Maxwell, W., and Venkataraman, K. 2018. Capital Commitment and Illiquidity in Corporate Bonds. The Journal of Finance 73: 1615-1661.

Biais, B., Hombert, J., and Weill, P.-O. 2017. Incentive constrained risk sharing, segmentation, and asset pricing. Working paper. National Bureau of Economic Research. 
Bo, B., and Victoria, I. 2015. Reaching for Yield in the Bond Market. The Journal of Finance 70: 1863-1902.

Boyarchenko, N., Eisenbach, T. M., Gupta, P., Shachar, O., and Van Tassel, P. 2018. Bankintermediated arbitrage. .

Brunnermeier, M. K., and Pedersen, L. H. 2008. Market liquidity and funding liquidity. The Review of Financial Studies 22: 2201-2238.

Brunnermeier, M. K., and Sannikov, Y. 2014. A Macroeconomic Model with a Financial Sector. American Economic Review 104 (February): 379-421.

Cai, F., Han, S., Li, D., and Li, Y. 2019. Institutional herding and its price impact: Evidence from the corporate bond market. Journal of Financial Economics 131: 139 - 167.

Carole, C.-F., Hendershott, T., Charles, J., Pam, M., and Mark, S. 2010. Time Variation in Liquidity: The Role of Market-Maker Inventories and Revenues. The Journal of Finance 65: 295-331.

Chen, H., Chen, Z., He, Z., Liu, J., and Xie, R. 2019. Pledgeability and asset prices: Evidence from the Chinese corporate bond markets. Working paper. University of Chicago, Booth School of Business.

Chen, H., Joslin, S., and Ni, S. X. 2018. Demand for Crash Insurance, Intermediary Constraints, and Risk Premia in Financial Markets. The Review of Financial Studies 32 (05): $228-265$.

Chen, L., Lesmond, D., and Wei, J. 2007. Corporate Yield Spreads and Bond Liquidity. The Journal of Finance 62: 119-149.

Choi, J., Shachar, O., and Shin, S. S. 2019. Dealer Liquidity Provision and the Breakdown of the Law of One Price: Evidence from the CDS-Bond Basis. Management Science 65: $4100-4122$.

Collin-Dufresne, P., Goldstein, R., and Martin, S. 2001. The determinants of credit spread changes. Journal of Finance 56: 2177-2207.

Constantinides, G. M., Jackwerth, J. C., and Savov, A. 2013. The Puzzle of Index Option Returns. The Review of Asset Pricing Studies 3 (05): 229-257.

Cragg, J. G., and Donald, S. G. 1993. Testing Identifiability and Specification in Instrumental Variable Models. Econometric Theory 9: 222-240.

Cui, R., Chen, H., He, Z., and Milbradt, K. 2017. Quantifying liquidity and default risks of corporate bonds over the business cycle. The Review of Financial Studies 31: 852-897.

de Jong, F., and Driessen, J. 2012. Liquidity Risk Premia in Corporate Bond Markets. Quarterly Journal of Finance 02: 1250006. 
Di Maggio, M., Kermani, A., and Song, Z. 2017. The value of trading relations in turbulent times. Journal of Financial Economics 124(2): 266-284.

Dick-Nielsen, J., Feldhütter, P., and Lando, D. 2012. Corporate bond liquidity before and after the onset of the subprime crisi. Journal of Financial Economic 103: 471 - 492.

Dick-Nielsen, J., and Rossi, M. 2018. The cost of immediacy for corporate bonds. The Review of Financial Studies 32: 1-41.

Du, W., Tepper, A., and Verdelhan, A. 2017. Deviations from Covered Interest Rate Parity. Journal of Finance forthcoming.

Duffie, D. 2010. Presidential address: Asset price dynamics with slow-moving capital. The Journal of finance 65: 1237-1267.

Duffie, D., Gârleanu, N., and Pedersen, L. 2005. Over-the-Counter Markets. Econometrica 73: $1815-1847$.

Ellul, A., Jotikasthira, C., and Lundblad, C. T. 2011. Regulatory pressure and fire sales in the corporate bond market. Journal of Financial Economics 101: 596 - 620.

Feldhütter, P. 2012. The Same Bond at Different Prices: Identifying Search Frictions and Selling Pressures. The Review of Financial Studies 25 (04): 1155-1206.

Fleckenstein, M., and Longstaff, F. 2019. Renting Balance Sheet Space: Intermediary Balance Sheet Rental Costs and the Valuation of Derivatives. working paper.

Friewald, N., and Nagler, F. 2019. Over-the-Counter Market Frictions and Yield Spread Changes. The Journal of Finance forthcoming.

Gabaix, X., Krishnamurthy, A., and Vigneron, O. 2007. Limits of Arbitrage: Theory and Evidence from the Mortgage-Backed Securities Market. Journal of Finance 2: 557-595.

Gao, P., Schultz, P., and Song, Z. 2017. Liquidity in a Market for Unique Assets: Specified Pool and TBA Trading in the Mortgage Backed Securities Market. Journal of Finance 72-3: 1119-1170.

Garleanu, N., and Pedersen, L. H. 2011. Margin-based asset pricing and deviations from the law of one price. The Review of Financial Studies 24: 1980-2022.

Goldstein, M. A., and Hotchkiss, E. S. 2019. Providing liquidity in an illiquid market: Dealer behavior in US corporate bonds. Journal of Financial Economics.

Gromb, D., and Vayanos, D. 2002. Equilibrium and welfare in markets with financially constrained arbitrageurs. Journal of Financial Economics 66: 361-407.

Gurkaynak, R., Sack, B., and Wright, J. 2007. The U.S. Treasury Yield Curve: 1961 to the Present. Journal of Monetary Economics 54(8): 2291-2304. 
He, Z., Kelly, B., and Manela, A. 2017. Intermediary asset pricing: New evidence from many asset classes. Journal of Financial Economics 126: 1-35.

He, Z., and Krishnamurthy, A. 2012. A Model of Capital and Crises. The Review of Economic Studies 79 (09): 735-777.

He, Z., and Krishnamurthy, A. 2013. Intermediary Asset Pricing. American Economic Review 103 (April): 732-70.

He, Z., and Krishnamurthy, A. 2018. Intermediary Asset Pricing and the Financial Crisis. Annual Review of Financial Economics 10: 173-197.

He, Z., and Milbradt, K. 2014. Endogenous Liquidity and Defaultable Bonds. Econometrica 82: $1443-1508$.

He, Z., and Xiong, W. 2012. Rollover risk and credit risk. The Journal of Finance 67: 391430.

Hendershott, T., and Menkveld, A. J. 2014. Price pressures. Journal of Financial Economics 114: $405-423$.

Ho, T., and Stoll, H. R. 1981. Optimal dealer pricing under transactions and return uncertainty. Journal of Financial Economics 9: 47 - 73.

Hu, X., Pan, J., and Wang, J. 2013. Noise as Information for Illiquidity. Journal of Finance 68: $2223-2772$.

Huang, J., and Shi, Z. 2014. A Resolution to the Equity-Credit Market Integration Puzzle. working paper.

Huang, J.-Z., and Huang, M. 2012. How Much of the Corporate-Treasury Yield Spread Is Due to Credit Risk?. The Review of Asset Pricing Studies 2 (11): 153-202.

Kondor, P., and Vayanos, D. 2019. Liquidity risk and the dynamics of arbitrage capital. The Journal of Finance 74: 1139-1173.

Kyle, A. S., and Xiong, W. 2001. Contagion as a wealth effect. The Journal of Finance 56: 1401-1440.

Leland, H. E. 1994. Corporate debt value, bond covenants, and optimal capital structure. The Journal of Finance 49: 1213-1252.

Leland, H. E., and Toft, K. B. 1996. Optimal capital structure, endogenous bankruptcy, and the term structure of credit spreads. The Journal of Finance 51: 987-1019.

Lin, H., Wang, J., and Wu, C. 2011. Liquidity risk and expected corporate bond returns. Journal of Financial Economics 99: 628 - 650. 
Longstaff, F. A., Mithal, S., and Neis, E. 2005. Corporate yield spreads: Default risk or liquidity? New evidence from the credit default swap market. The Journal of Finance 60: 2213-2253.

Manconi, A., Massa, M., and Yasuda, A. 2012. The role of institutional investors in propagating the crisis of 2007-2008. Journal of Financial Economics 104: 491 - 518 Market Institutions, Financial Market Risks and Financial Crisis.

Manzi, J., Berezina, D., and Adelson, M. 2016. Synthetic CMBS Primer. Nomura Fixed Income Research.

Merton, R. 1974. On the pricing of corporate debt: the risk structure of interest rates. Journal of Finance 29: 449-470.

Mitchell, M., Pedersen, L. H., and Pulvino, T. 2007. Slow moving capital. American Economic Review 97: 215-220.

Montiel-Olea, J. L., and Pflueger, C. 2013. A Robust Test for Weak Instruments. Journal of Business \& Economic Statistics 31: 358-369.

Nelson, C. R., and Siegel, A. F. 1987. Parsimonious Modeling of Yield Curves. Journal of Business 60: 473-490.

Newey, W., and West, K. 1987. A Simple, Positive Semi-Definite, Heteroskedasticity and Autocorrelation Consistent Covariance Matrix. Econometrica 55: 703-708.

Pástor, L., and Stambaugh, R. F. 2003. Liquidity Risk And Expected Stock Returns. Journal of Political Economy 111: 642-685.

Pflueger, C., and Wang, S. 2015. A robust test for weak instruments in Stata. Stata Journal 15: $216-225(10)$.

Schaefer, S. M., and Strebulaev, I. A. 2008. Structural models of credit risk are useful: Evidence from hedge ratios on corporate bonds. Journal of Financial Economics 90: 1 19.

Schultz, P. 2017. Inventory management by corporate bond dealers. Working Paper University of Notre Dame.

Shan, S. C., Tang, D. Y., and Yan, H. 2016. Credit Default Swaps and Bank Regulatory Capital. Working Paper.

Siriwardane, E. N. 2019. Limited Investment Capital and Credit Spreads. The Journal of Finance 0.

Song, Z., and Zhu, H. 2018. Quantitative easing auctions of Treasury bonds. Journal of Financial Economics 128: 103 - 124. 
Stock, J. H., and Wright, J. H. 2000. GMM with Weak Identification. Econometrica 68: 1055-1096.

Stock, J. H., and Yogo, M. 2005. Testing for Weak Instruments in Linear IV Regression Cambridge University Press pp. 80-108.

Svensson, L. 1994. Estimating and Interpreting Forward Rates: Sweden 1992-1994. National Bureau of Economic Research Working Paper 4871.

U.S. SEC 1992. The corporate bond markets: Structure, pricing and trading. U.S. Securities and Exchange Commission: Division of Market Regulation Washington, D.C. 


\section{Figure A.1: Summary of Institutional Holdings}
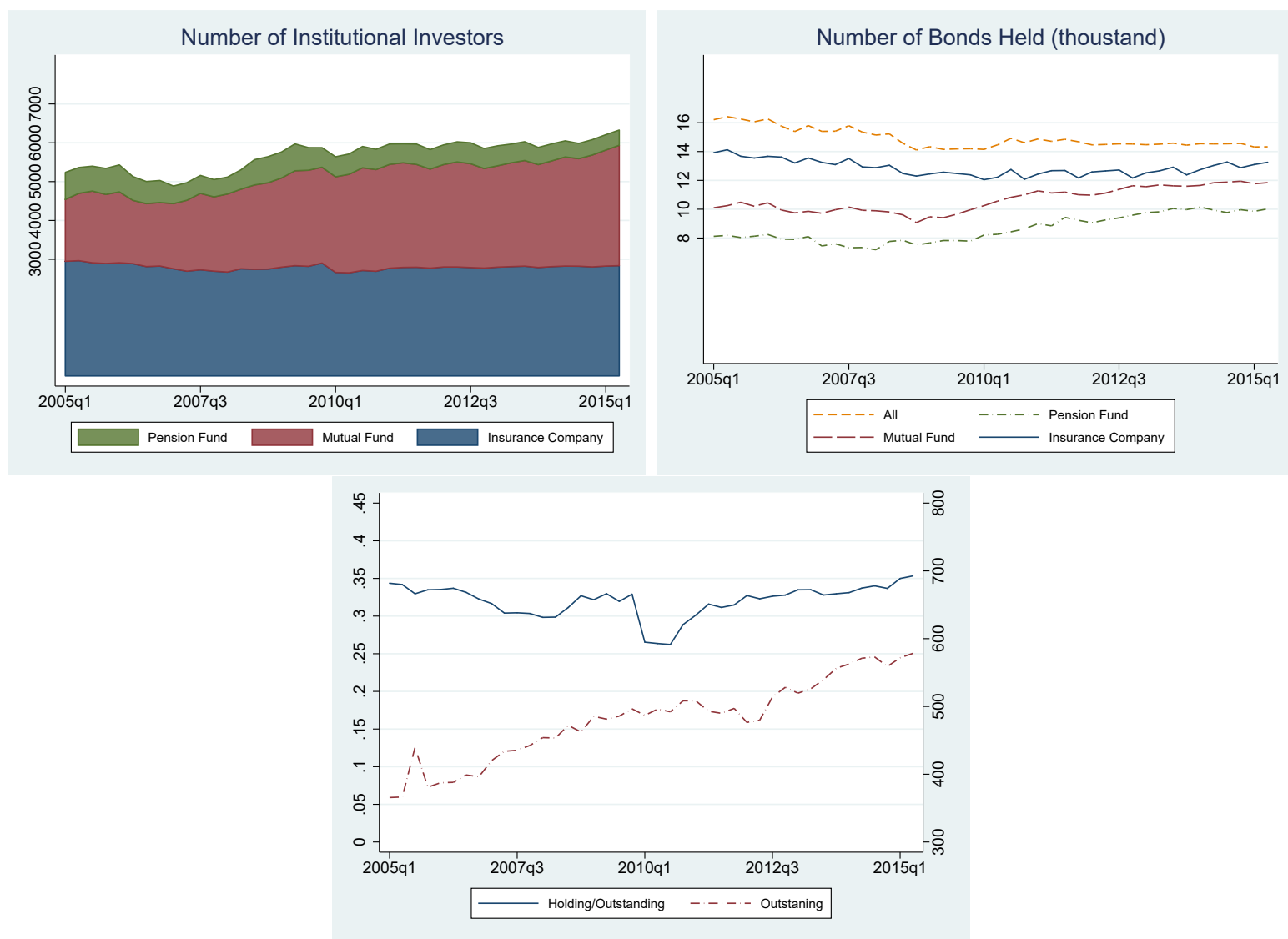

Note: This figure plots quarterly time series, based on eMAXX data of institutional holdings, of the number of institutional investors (top left panel) and the number of bonds in thousands (top right panel), by insurance companies, mutual funds, pension funds, and all institutions separately, as well as an average bond's outstanding balance (in \$millions) and ratio of total holding amount by all institutions to outstanding balance (bottom panel). The number of bonds held by all institutions is lower than the sum of the number of bonds held by insurance companies, mutual funds, and pension funds because different institutions can hold the same bond. The average bond's total holding amount is calculated by first summing the holding amounts by all institutions for each bond in each quarter and then taking an average across all the bonds in each quarter. The average bonds' outstanding balance is computed by taking the average of outstanding balance across all the bonds in each quarter. The average bond's ratio of holding to outstanding is computed by dividing its total holding amount by outstanding balance in each quarter. The sample period is from 2005:Q1 through 2015:Q2. 


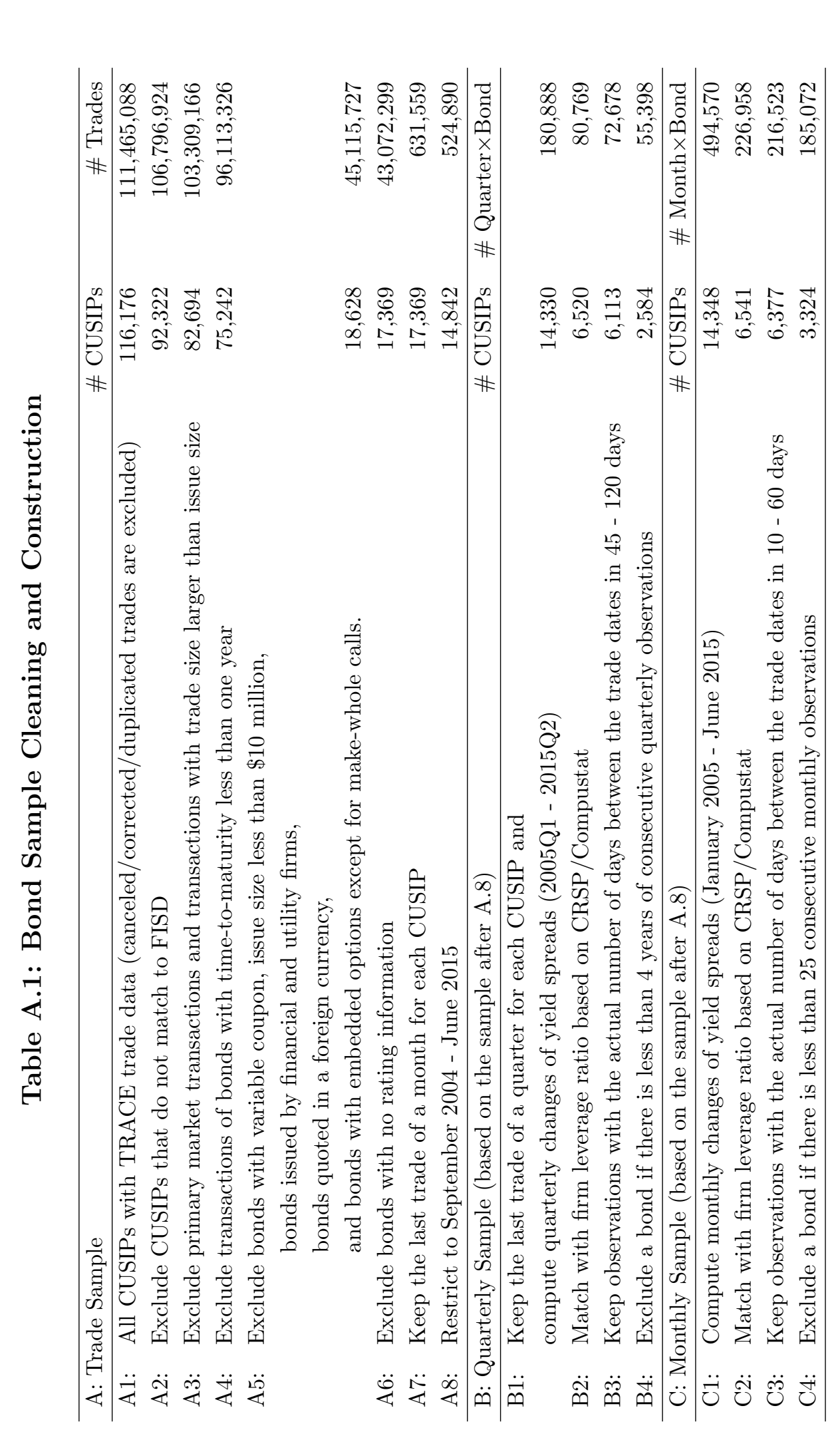

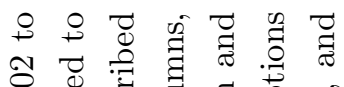

实焉

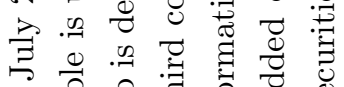

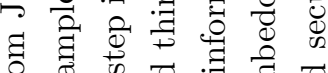

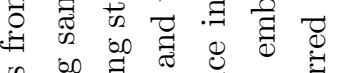
.

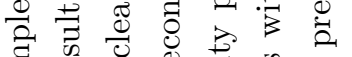
สี

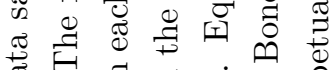

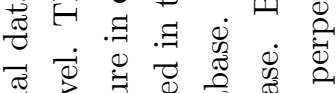
要 ర) E

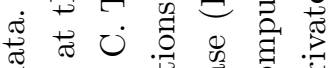

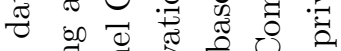

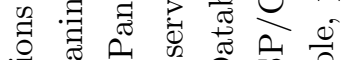
.

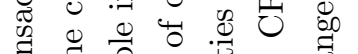
苛

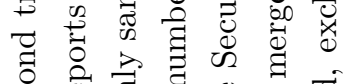
○)

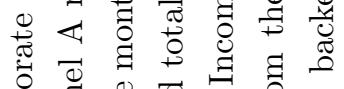

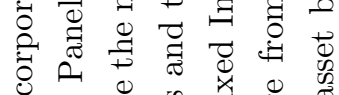

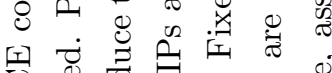

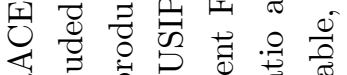

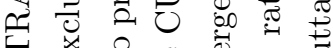

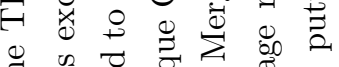
娄 फे

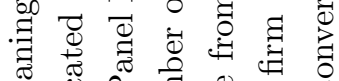
芯

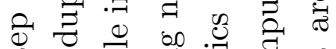

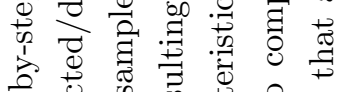

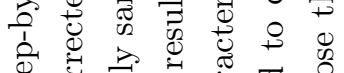
虫

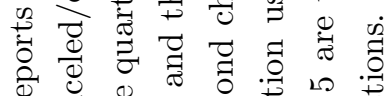

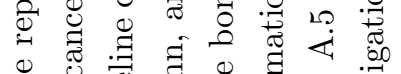

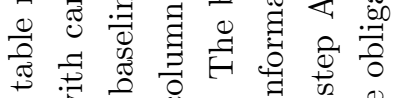

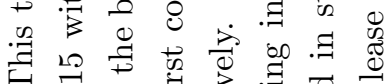

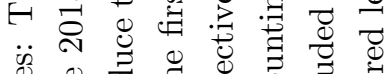

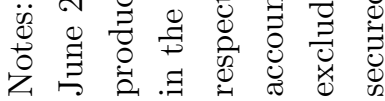


Table A.2: Summary of Institutional Holdings

\begin{tabular}{|c|c|c|c|c|c|c|c|}
\hline & mean & $\mathrm{sd}$ & $\min$ & $\mathrm{p} 25$ & p50 & $\mathrm{p} 75$ & $\max$ \\
\hline \multicolumn{8}{|c|}{ A: Number of Institutional Investors } \\
\hline Insurance Company & 2797 & 74 & 2653 & 2756 & 2801 & 2826 & 2965 \\
\hline Mutual Fund & 2345 & 436 & 1593 & 1912 & 2504 & 2672 & 3099 \\
\hline Pension Fund & 529 & 92 & 392 & 453 & 515 & 582 & 696 \\
\hline All & 5670 & 392 & 4886 & 5340 & 5842 & 5971 & 6327 \\
\hline \multicolumn{8}{|c|}{ B: Number of Bonds } \\
\hline Insurance Company & 12873 & 525 & 12049 & 12477 & 12748 & 13249 & 14125 \\
\hline Mutual Fund & 10652 & 843 & 9072 & 9925 & 10523 & 11561 & 11943 \\
\hline Pension Fund & 8629 & 945 & 7189 & 7826 & 8254 & 9590 & 10150 \\
\hline All & 14910 & 673 & 14109 & 14465 & 14579 & 15392 & 16424 \\
\hline \multicolumn{8}{|c|}{ C: Aggregate Holding Amount (\$trillion) } \\
\hline Insurance Company & 1.02 & 0.16 & 0.74 & 0.91 & 0.96 & 1.16 & 1.30 \\
\hline Mutual Fund & 0.67 & 0.27 & 0.28 & 0.39 & 0.70 & 0.91 & 1.13 \\
\hline Pension Fund & 0.11 & 0.02 & 0.07 & 0.10 & 0.11 & 0.12 & 0.15 \\
\hline All & 1.80 & 0.41 & 1.28 & 1.40 & 1.68 & 2.18 & 2.54 \\
\hline \multicolumn{8}{|c|}{ D: Average Bond Holding Amount and Outstanding Balance (\$million) } \\
\hline Average Bond Holding Amount & 116.54 & 27.18 & 80.94 & 87.99 & 114.74 & 143.35 & 162.04 \\
\hline Average Bond Outstanding Balance & 480.32 & 59.80 & 365.62 & 439.65 & 486.27 & 519.65 & 578.31 \\
\hline Average Bond Holding/Outstanding & 0.32 & 0.02 & 0.26 & 0.31 & 0.33 & 0.33 & 0.35 \\
\hline
\end{tabular}

Notes: This table reports summary statistics of quarterly time series, based on eMAXX data of institutional holdings, of the number of institutional investors (in panel A), the number of bonds (in panel B), and aggregate holding amount in \$trillions of principal value (in panel C), by insurance companies, mutual funds, pension funds, and all institutions separately, as well as an average bond's holding amount (in \$millions), outstanding balance (in \$millions) and ratio of holding amount by all institutions to outstanding balance (in panel D). The number of bonds held by all institutions is lower than the sum of the number of bonds held by insurance companies, mutual funds, and pension funds because different institutions can hold the same bond. The average bond's total holding amount is calculated by first summing the holding amounts by all institutions for each bond in each quarter and then taking an average across all the bonds in each quarter. The average bonds' outstanding balance is computed by taking the average of outstanding balance across all the bonds in each quarter. The average bond's ratio of holding to outstanding is computed by dividing its total holding amount by outstanding balance in each quarter. The sample period is from 2005:Q1 through 2015:Q2. 
Table A.3: Summary of Yield Spreads and Returns of Non-Corporate-Credit Assets

\begin{tabular}{|c|c|c|c|c|c|c|}
\hline & $\mathrm{N}$ & mean & $\mathrm{sd}$ & $\mathrm{p} 25$ & $\mathrm{p} 50$ & $\mathrm{p} 75$ \\
\hline \multicolumn{7}{|c|}{ A: Agency MBS (in BPs) } \\
\hline FN30y & 42 & 15.76 & 21.17 & -4.48 & 14.96 & 34.40 \\
\hline FN15y & 42 & 16.07 & 23.75 & -3.65 & 10.04 & 31.73 \\
\hline FG30y & 42 & 18.79 & 22.73 & -2.84 & 17.63 & 35.30 \\
\hline FG15y & 42 & 22.31 & 22.92 & 4.41 & 17.48 & 35.59 \\
\hline \multicolumn{7}{|c|}{ B: Non-agency CMBS (in BPs) } \\
\hline Duper & 39 & 153.62 & 168.56 & 73.00 & 99.00 & 185.00 \\
\hline $\mathrm{AM}$ & 39 & 296.38 & 402.65 & 63.00 & 133.00 & 341.00 \\
\hline AJ & 39 & 439.03 & 608.24 & 121.00 & 210.00 & 450.00 \\
\hline \multicolumn{7}{|c|}{ C: ABS (in BPs) } \\
\hline Credit Card Loan 5y & 40 & 79.08 & 67.93 & 47.00 & 54.00 & 63.50 \\
\hline Auto Loan 3y: AAA & 37 & 50.57 & 67.21 & 19.00 & 27.00 & 36.00 \\
\hline Auto Loan 3y: A & 36 & 121.36 & 136.64 & 56.50 & 74.00 & 122.50 \\
\hline Auto Loan: 3y BBB & 34 & 154.74 & 136.71 & 100.00 & 121.00 & 175.00 \\
\hline \multicolumn{7}{|c|}{ D: S\&P 500 index options (in percentage) } \\
\hline Call: 0.90 & 85 & 0.09 & 4.41 & -2.04 & 0.51 & 2.28 \\
\hline Call: 0.95 & 85 & 0.02 & 4.30 & -1.84 & 0.30 & 2.05 \\
\hline Call: ATM & 85 & -0.12 & 4.14 & -1.75 & 0.04 & 1.65 \\
\hline Call: 1.05 & 85 & -0.26 & 3.94 & -1.77 & -0.14 & 1.71 \\
\hline Call: 1.10 & 85 & -0.49 & 3.64 & -1.78 & -0.35 & 0.81 \\
\hline Put: 0.90 & 85 & -0.89 & 7.79 & -4.56 & -1.95 & 1.63 \\
\hline Put: 0.95 & 85 & -0.74 & 6.95 & -3.96 & -1.58 & 1.37 \\
\hline Put: ATM & 85 & -0.54 & 6.28 & -3.34 & -1.12 & 1.78 \\
\hline Put: 1.05 & 85 & -0.38 & 5.71 & -3.00 & -0.86 & 1.68 \\
\hline Put: 1.10 & 85 & -0.32 & 5.37 & -2.82 & -0.94 & 1.70 \\
\hline
\end{tabular}

Note: This table reports summary statistics of quarterly time series of option-adjusted spreads of agency MBS, yield spreads of non-agency CMBS, and yield spreads of ABS, all in basis points, in panels A, B, and $\mathrm{C}$, respectively, as well as summary statistics of monthly series of (unannualized) one-month return in percent of leverage-adjusted S\&P 500 index option portfolios. The series of yield spreads are provided by major Wall Street dealers, whereas the option returns are those used in Constantinides, Jackwerth, and Savov (2013). The sample period of yield spreads is 2005:Q1 - 2015:Q2 overall, with variation across different series depending on data availability. The sample period is January 2005 - January 2012 for options. 


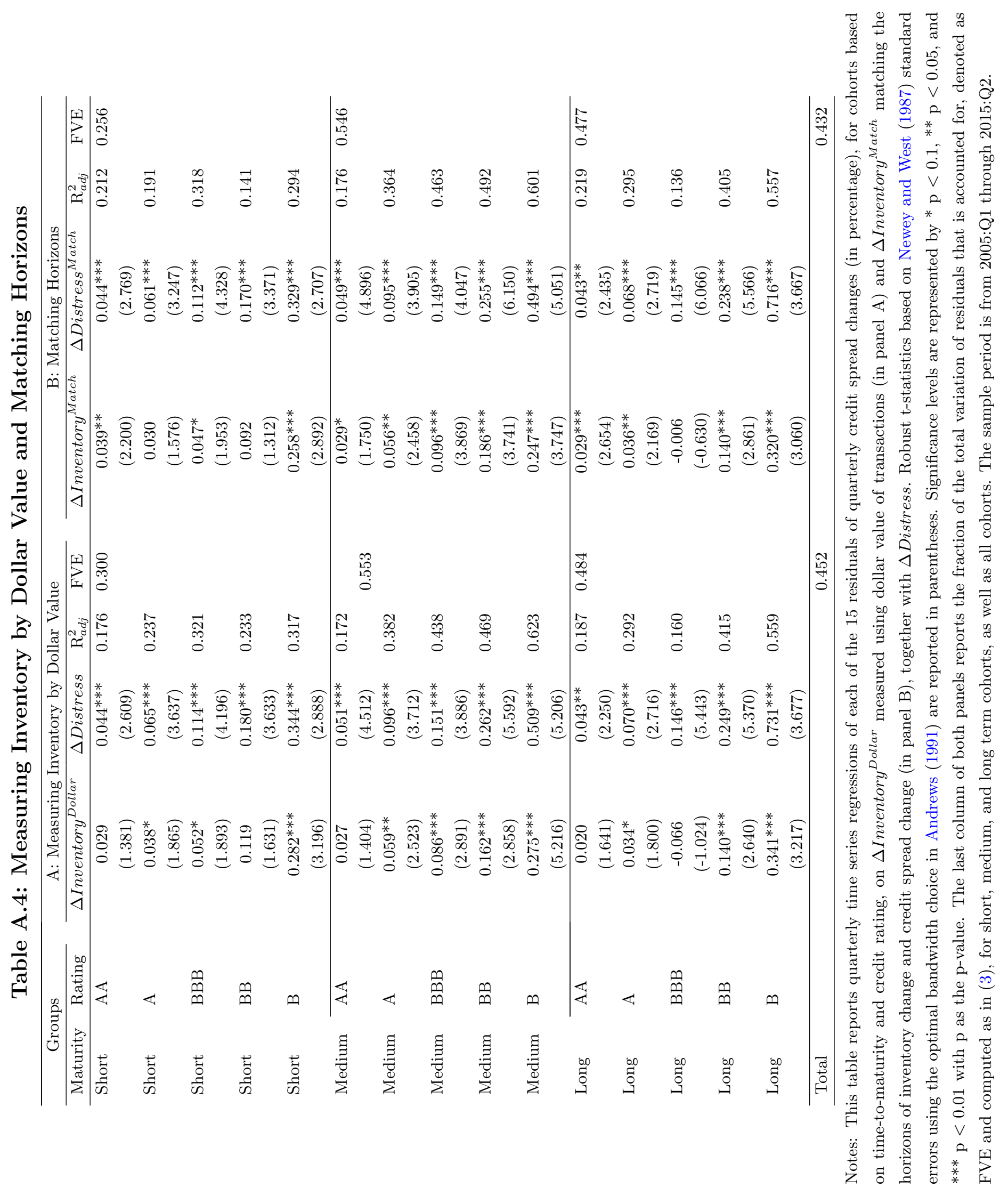




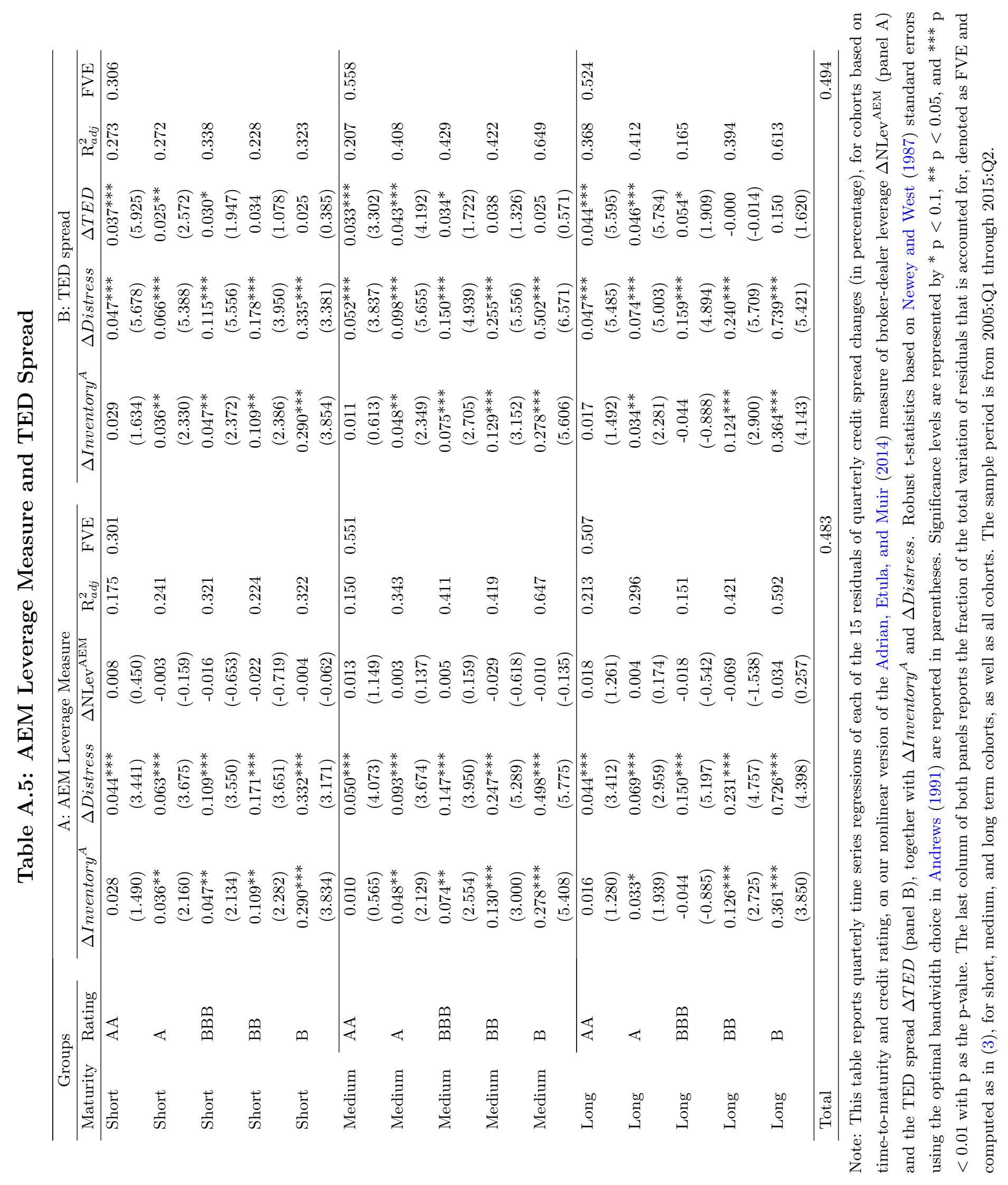




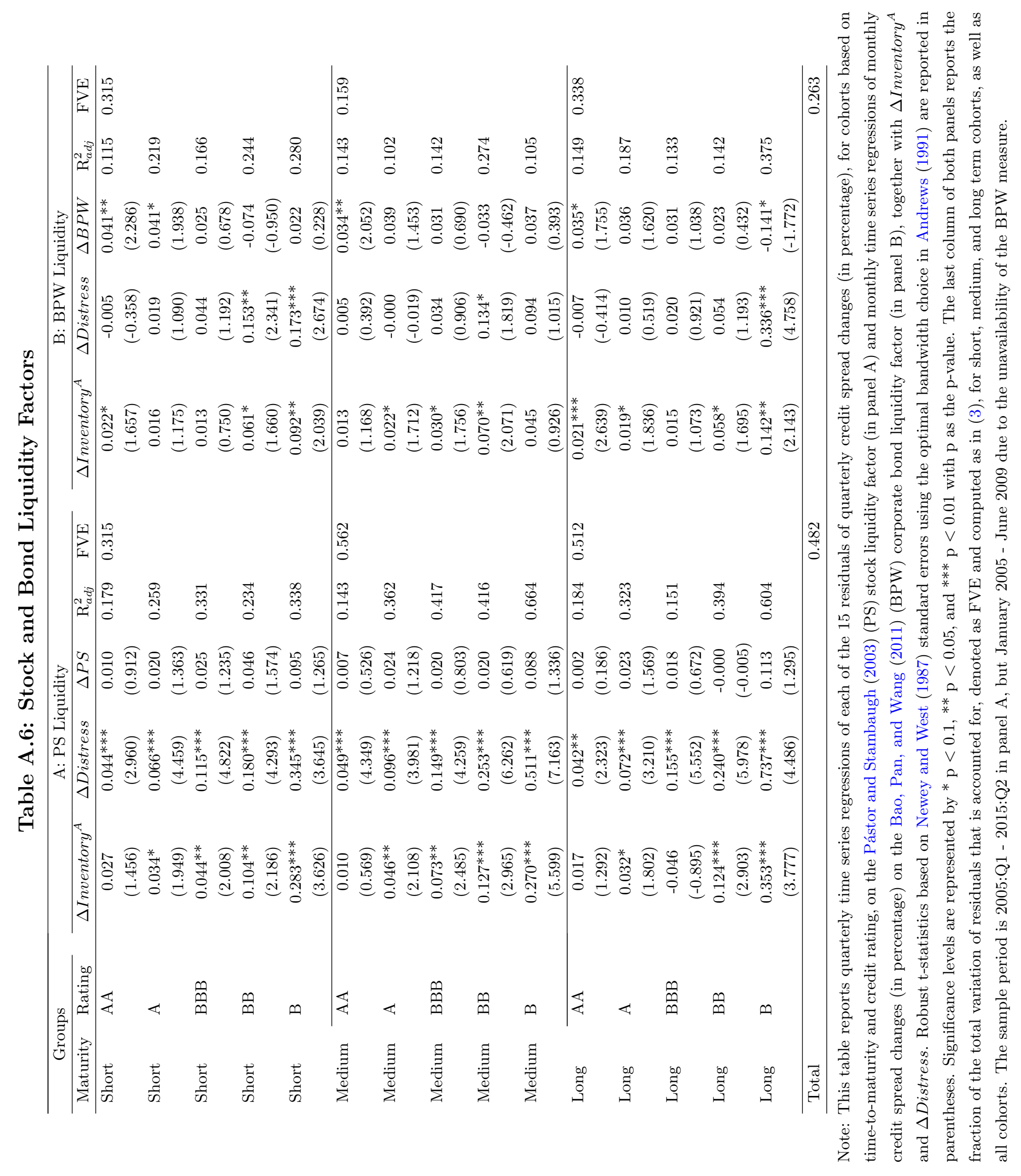


Table A.7: Excluding the 2008 Crisis

\begin{tabular}{|c|c|c|c|c|c|c|c|}
\hline \multicolumn{2}{|c|}{ Groups } & \multicolumn{2}{|c|}{ A: PC } & \multicolumn{4}{|c|}{ B: Regression of Residuals } \\
\hline Maturity & Rating & First & Second & $\Delta$ Inventory $^{A}$ & $\Delta$ Distress & $\mathrm{R}_{a d j}^{2}$ & FVE \\
\hline Short & $\mathrm{AA}$ & 0.062 & -0.019 & $\begin{array}{c}0.020 \\
(0.918)\end{array}$ & $\begin{array}{c}0.042^{* * *} \\
(3.399)\end{array}$ & 0.173 & 0.268 \\
\hline Short & A & 0.079 & -0.026 & $\begin{array}{c}0.030 \\
(1.560)\end{array}$ & $\begin{array}{c}0.051^{* * *} \\
(3.641)\end{array}$ & 0.208 & \\
\hline Short & BBB & 0.125 & -0.022 & $\begin{array}{l}0.042^{*} \\
(1.744)\end{array}$ & $\begin{array}{l}0.92^{* * *} \\
(3.573)\end{array}$ & 0.266 & \\
\hline Short & $\mathrm{BB}$ & 0.154 & -0.136 & $\begin{array}{c}0.092^{* *} \\
(2.283)\end{array}$ & $\begin{array}{c}0.065 \\
(1.283)\end{array}$ & 0.142 & \\
\hline Short & B & 0.459 & -0.394 & $\begin{array}{c}0.264^{* * *} \\
(3.097)\end{array}$ & $\begin{array}{l}0.273^{* *} \\
(2.243)\end{array}$ & 0.277 & \\
\hline Medium & AA & 0.05 & -0.061 & $\begin{array}{l}-0.008 \\
(-0.476)\end{array}$ & $\begin{array}{c}0.041^{* * *} \\
(3.234)\end{array}$ & 0.132 & 0.583 \\
\hline Medium & A & 0.1 & -0.02 & $\begin{array}{c}0.035 \\
(1.475)\end{array}$ & $\begin{array}{c}0.098^{* * *} \\
(5.975)\end{array}$ & 0.396 & \\
\hline Medium & BBB & 0.159 & -0.023 & $\begin{array}{l}0.059^{*} \\
(1.829)\end{array}$ & $\begin{array}{c}0.146^{* * *} \\
(5.086)\end{array}$ & 0.421 & \\
\hline Medium & $\mathrm{BB}$ & 0.172 & 0.099 & $\begin{array}{c}0.087^{* *} \\
(2.492)\end{array}$ & $\begin{array}{c}0.170^{* * *} \\
(5.737)\end{array}$ & 0.463 & \\
\hline Medium & B & 0.443 & 0.041 & $\begin{array}{c}0.249^{* * *} \\
(4.355)\end{array}$ & $\begin{array}{c}0.461^{* * *} \\
(6.453)\end{array}$ & 0.651 & \\
\hline Long & $\mathrm{AA}$ & 0.055 & 0.006 & $\begin{array}{c}0.008 \\
(0.671)\end{array}$ & $\begin{array}{c}0.055^{* * *} \\
(6.002)\end{array}$ & 0.330 & 0.542 \\
\hline Long & A & 0.077 & -0.009 & $\begin{array}{l}0.030^{*} \\
(1.792)\end{array}$ & $\begin{array}{l}0.73^{* * *} \\
(5.351)\end{array}$ & 0.395 & \\
\hline Long & $\mathrm{BBB}$ & 0.069 & 0.88 & $\begin{array}{l}-0.072 \\
(-1.343)\end{array}$ & $\begin{array}{c}0.156^{* * *} \\
(5.183)\end{array}$ & 0.140 & \\
\hline Long & $\mathrm{BB}$ & 0.181 & 0.102 & $\begin{array}{c}0.085^{* *} \\
(2.086)\end{array}$ & $\begin{array}{c}0.164^{* * *} \\
(5.032)\end{array}$ & 0.366 & \\
\hline Long & B & 0.656 & 0.156 & $\begin{array}{c}0.309^{* * *} \\
(3.503)\end{array}$ & $\begin{array}{c}0.699^{* * * *} \\
(6.294)\end{array}$ & 0.650 & \\
\hline Pct Expla & ned & 0.798 & 0.082 & & & & 0.477 \\
\hline
\end{tabular}

Note: This table reports results using 15 cohorts based on time-to-maturity and credit rating excluding the 2008 crisis period, defined as 2007:Q3 - 2009:Q1. Panel A reports the loadings of the first two PCs on the 15 regression residuals and the fraction of total variation these two PCs account for. Panel B reports quarterly time series regressions of each of the 15 residuals of quarterly credit spread changes (in percentage) on $\Delta$ Inventory $^{A}$ and $\Delta$ Distress, with robust t-statistics based on Newey and West (1987) standard errors using the optimal bandwidth choice in Andrews (1991) reported in parentheses. Significance levels are represented by ${ }^{*} \mathrm{p}<0.1,{ }^{* *} \mathrm{p}<0.05$, and ${ }^{* * *} \mathrm{p}<0.01$ with $\mathrm{p}$ as the $\mathrm{p}$-value. The last column of panel $\mathrm{B}$ reports the fraction of the total variation of residuals that is accounted for by the two intermediary factors, denoted as FVE and computed as in (3) for short, medium, and long term bonds, as well as all bonds. 
Table A.8: Correlations of Dealer Inventories of HY and IG Bonds

\begin{tabular}{|c|c|c|c|}
\hline \multicolumn{4}{|c|}{ A: Raw Change } \\
\hline & $\Delta$ Inventory $^{A}$ & $\Delta$ Inventory $^{H Y}$ & $\Delta$ Inventory $^{I G}$ \\
\hline$\Delta$ Inventory $^{A}$ & 1.0000 & & \\
\hline$\Delta$ Inventory ${ }^{H Y}$ & $0.6854^{*}$ & 1.0000 & \\
\hline$\Delta$ Inventory ${ }^{I G}$ & $0.5718^{*}$ & -0.2054 & 1.0000 \\
\hline \multicolumn{4}{|c|}{ B: Percentage Change } \\
\hline & $\Delta$ Inventory $^{A}$ & $\Delta$ Inventory $^{H Y}$ & $\Delta$ Inventory $^{I G}$ \\
\hline$\Delta$ Inventory $^{A}$ & 1.0000 & & \\
\hline$\Delta$ Inventory ${ }^{H Y}$ & $0.7135^{*}$ & 1.0000 & \\
\hline$\Delta$ Inventory ${ }^{I G}$ & $0.7148^{*}$ & 0.0200 & 1.0000 \\
\hline
\end{tabular}

Note: This table reports quarterly time series correlations of three different measures related to dealer inventory, $\Delta$ Inventor $^{A}, \Delta$ Inventory $^{H Y}$, and $\Delta$ Inventory $^{I G}$. Both simple changes (in panel A) and percentage changes (in panel B) are included. Significance levels are represented by ${ }^{*} \mathrm{p}<0.1,{ }^{* *} \mathrm{p}<0.05$, and ${ }^{* * *} \mathrm{p}$ $<0.01$ with $\mathrm{p}$ as the p-value. The sample period is from 2005:Q1 through 2015:Q2. 


\section{Table A.9: Summary of Institutional Holdings by Rating Categories}

\begin{tabular}{|c|c|c|c|c|c|c|}
\hline & \multicolumn{2}{|c|}{ Insurance Companies } & \multicolumn{2}{|c|}{ Mutual Funds } & \multicolumn{2}{|c|}{ Pension Funds } \\
\hline & Amount (\$billion) & Fraction (\%) & Amount (\$billion) & Fraction (\%) & Amount (\$billion) & Fraction $(\%)$ \\
\hline AAA & 17.18 & 1.69 & 16.72 & 2.71 & 3.75 & 3.24 \\
\hline $\mathrm{AA}$ & 76.45 & 7.37 & 37.74 & 6.05 & 6.24 & 5.79 \\
\hline A & 368.03 & 35.45 & 128.05 & 18.27 & 23.57 & 21.23 \\
\hline BBB & 435.67 & 41.91 & 193.01 & 26.76 & 34.99 & 31.39 \\
\hline $\mathrm{BB}$ & 79.40 & 7.73 & 103.45 & 14.77 & 14.95 & 13.54 \\
\hline B & 33.92 & 3.30 & 121.19 & 17.68 & 15.76 & 14.24 \\
\hline $\mathrm{CCC}$ & 24.84 & 2.54 & 90.19 & 13.76 & 11.49 & 10.56 \\
\hline Total & 1035.48 & & 690.34 & & 110.75 & \\
\hline
\end{tabular}

Note: This table reports the average (over time) amount in \$billions and fraction in percent of the eMAXX quarterly corporate bond holdings of insurance companies, mutual funds, and pension funds, respectively, broken down into seven rating groups. The sample period is from 2005:Q1 through 2015:Q2. 